\title{
THE NEUMANN SIEVE PROBLEM AND DIMENSIONAL REDUCTION: A MULTISCALE APPROACH
}

\author{
NADIA ANSINI, JEAN-FRANÇOIS BABADJIAN \& CATERINA IDA ZEPPIERI
}

\begin{abstract}
We perform a multiscale analysis for the elastic energy of a $n$-dimensional bilayer thin film of thickness $2 \delta$ whose layers are connected through an $\varepsilon$-periodically distributed contact zone. Describing the contact zone as a union of $(n-1)$-dimensional balls of radius $r \ll \varepsilon$ (the holes of the sieve) and assuming that $\delta \ll \varepsilon$, we show that the asymptotic memory of the sieve (as $\varepsilon \rightarrow 0$ ) is witnessed by the presence of an extra interfacial energy term. Moreover we find three different limit behaviors (or regimes) depending on the mutual vanishing rate of $\delta$ and $r$. We also give an explicit nonlinear capacitary-type formula for the interfacial energy density in each regime.
\end{abstract}

KEYWORDS: $\Gamma$-convergence, dimension reduction, sieve problem, nonlinear capacity.

2000 Mathematics Subject Classification: 49J45, 74K35, 74K15, 74B20, 74G65, 35B40.

\section{INTRODUCTION}

For an ever increasing variety of applications, an interesting problem to be explored is to model the debonding of a thin film from a substrate.

If we consider a stretched film bonded to an infinite rigid substrate, the elastic energy of this film scales as its thickness. If the film debonds from the substrate, on one hand its elastic energy tends to zero, while on the other hand this creates a new surface and then an interfacial energy independent of the thickness.

In [6] Bhattacharya, Fonseca and Francfort examine, among other, the asymptotic behavior of a bilayer thin film allowing for the possibility of a debonding at the interface, but penalizing it postulating an interfacial energy which scales as the overall thickness of the film to some exponent. Thus the energy they consider consists of the elastic energy of the two layers and the interfacial energy with penalized debonding.

In this paper we deal with thin films connected by a hyperplane (sieve plane) through a periodically distributed contact zone. Thus we see the debonding as the effect of the weak interaction of the two thin films through this contact zone and we recover the interfacial energy term by a limit procedure.

Since we are mainly interested in describing the interaction phenomenon due to the presence of the sieve, we make a simplification choosing two thin films having the same elastic properties (for a generalization to the case of two different materials interacting, we refer the reader to [2]).

Consider a nonlinear elastic $n$-dimensional bilayer thin film of thickness $2 \delta$ with layers connected through $(n-1)$-dimensional balls $B_{r}^{n-1}\left(x_{i}^{\varepsilon}\right)$ centered in $x_{i}^{\varepsilon}:=i \varepsilon, i \in \mathbb{Z}^{n-1}$ and with radius $r>0$. Thus the investigated elastic body occupies the reference configuration parametrized as

$$
\Omega_{\varepsilon, r}^{\delta}:=\omega^{+\delta} \cup \omega^{-\delta} \cup\left(\omega_{\varepsilon, r} \times\{0\}\right)
$$

where $\omega$ is a bounded open subset of $\mathbb{R}^{n-1}, \omega^{+\delta}:=\omega \times(0, \delta), \omega^{-\delta}:=\omega \times(-\delta, 0)$ and $\omega_{\varepsilon, r}:=$ $\bigcup_{i \in \mathbb{Z}^{n-1}} B_{r}^{n-1}\left(x_{i}^{\varepsilon}\right) \cap \omega$ (see Figure 1).

In the nonlinear membrane theory setting the (scaled) elastic energy associated to the material modelled by $\Omega_{\varepsilon, r}^{\delta}$ is given by

$$
\frac{1}{\delta} \int_{\Omega_{\varepsilon, r}^{\delta}} W(D u) d x
$$




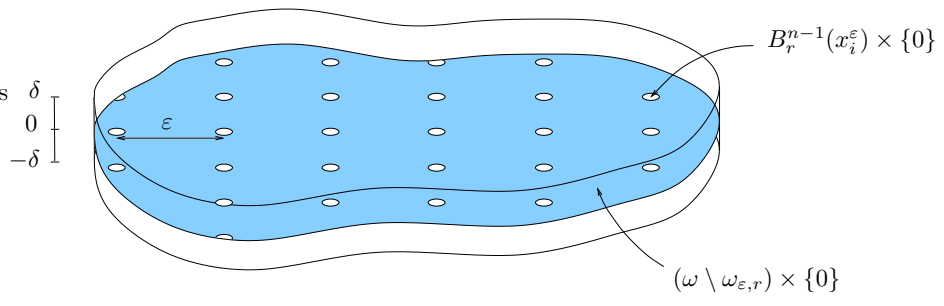

Figure 1. The domain $\Omega_{\varepsilon, r}^{\delta}$.

where $u: \Omega_{\varepsilon, r}^{\delta} \rightarrow \mathbb{R}^{m}$ is the deformation field and $W$ is the stored energy density.

The $\Gamma$-convergence approach has been used successfully in recent years to rigorously obtain limit models for various dimensional reductional problems (see for example $[7,12,13,23,29]$ ).

In this paper we study the multiscale asymptotic behavior of (1.1) via $\Gamma$-convergence, as $\varepsilon, \delta$ and $r$ tend to zero, assuming that $\delta=\delta(\varepsilon), r=r(\varepsilon, \delta)$ and where $W: \mathbb{R}^{m \times n} \rightarrow[0,+\infty)$ is a Borel function satisfying a growth condition of order $p$, with $1<p<n-1$. The case $p=n-1$ requires a further appropriate analysis and it cannot be easily derived from $p<n-1$ by slight changes. Unfortunately, three dimensional linearized elasticity falls into this framework.

Since the sieve $\left(\omega \backslash \omega_{\varepsilon, r}\right) \times\{0\}$ is not a part of the domain $\Omega_{\varepsilon, r}^{\delta}$, for any fixed $\varepsilon, \delta, r>0$ we have no information on the admissible deformation across part of the mid-section $\omega \times\{0\}$. This possible lack of regularity might produce, in the limit, the above mentioned debonding and correspondingly an interfacial energy depending on the jump of the limit deformation. Moreover, we expect that this interfacial energy will depend on the scaling of the radius of the connecting zones with respect to the period of their distribution and the thickness of the thin film.

The cases $\delta=1$ and $\delta=\varepsilon$ have been studied by Ansini [2] who proved that, to recover a non trivial limit model; i.e., to obtain a limit model remembering the presence of the sieve, the meaningful radius (or critical size) of the contact zones must be of order $\varepsilon^{(n-1) /(n-p)}$ and $\varepsilon^{n /(n-p)}$, respectively. In fact a different choice should lead in the limit to two decoupled problems (if $r$ tends to zero faster than the critical size) or to the same result that is obtained without the presence of connecting zones in the mid-section (if $r$ tends to zero more slowly than the critical size).

The proofs of the $\Gamma$-convergence results in [2] (see Theorems 3.2 and 8.2 therein) are based on a technical lemma ([2], Lemma 3.4) that allows to modify a sequence of deformations $u_{\varepsilon}$ with equi-bounded energy, on a suitable $n$-dimensional spherical annuli surrounding the balls $B_{r}^{n-1}\left(x_{i}^{\varepsilon}\right)$ without essentially changing their energies, and to study the behavior of the energies along the new modified sequence. Both in the case $\delta=1$ and $\delta=\varepsilon$ the $\Gamma$-limits consist of three terms. The first two terms represent the contribution of the new sequence far from the balls $B_{r}^{n-1}\left(x_{i}^{\varepsilon}\right)$; more precisely, they are the $\Gamma$-limits of two problems defined separately on the upper and lower part (with respect to the 'sieve plane') of the considered domain. The third term describes the contribution near the balls $B_{r}^{n-1}\left(x_{i}^{\varepsilon}\right)$ through a nonlinear capacitary-type formula that is the same for both $\delta=1$ and $\delta=\varepsilon$. The equality of the two formulas is due to the fact that the radii of the annuli suitably chosen to separate the two contributions are less than $c \varepsilon$, with $c$ an arbitrary small positive constant. In fact as a consequence, all constructions can be performed in the interior of the domain, and the same procedure yielding the nonlinear capacitarytype formula, applies for $\delta=1$ and for $\delta=\varepsilon$ as well. The cases $\varepsilon \sim \delta$ and $\varepsilon \ll \delta$ can be treated in the same way.

This approach follows the method introduced by Ansini-Braides in $[3,4]$ where the asymptotic behavior of periodically perforated nonlinear domains has been studied; in particular, Lemma 3.4 in [2] is a suitable variant, for the sieve problem, of Lemma 3.1 in [3]. 
For other problems related to this subject, we refer the reader to Attouch-Damlamian-Murat-Picard [21], [24], [25], Attouch-Picard [5], Conca [16, 17, 18], Del Vecchio [22] and Sanchez-Palencia [27, 26, 28], among others.

In this paper we focus our attention on the case $\delta=\delta(\varepsilon) \ll \varepsilon$. As in [2], we expect the existence of a critical radius $r=r(\varepsilon, \delta) \ll \varepsilon$ for which the limit model is nontrivial but now we expect also to find different limit regimes depending on the mutual vanishing rate of $r$ and $\delta$. Moreover Lemma 3.4 in [2] cannot be directly applied to our setting since the spherical annuli surrounding the connecting zones $B_{r}^{n-1}\left(x_{i}^{\varepsilon}\right)$ as above, are well contained in a strip of thickness $c \varepsilon$ but not in $\Omega_{\varepsilon, r}^{\delta}(\delta \ll \varepsilon)$. However, we are able to modify Lemma 3.4 in [2] by considering, instead of spherical annuli, suitable cylindrical annuli of thickness of order $\delta$ (see Lemma 4.2 and Lemma 4.4).

As a consequence, also in this case the asymptotic analysis of (1.1) as $\varepsilon, \delta$ and $r$ tend to zero can be carried on studying separately the energy contributions far from and close to $B_{r}^{n-1}\left(x_{i}^{\varepsilon}\right)$; we get three terms in the limit. The first two terms still describe the contribution 'far' from the connecting zones; i.e., they are the $\Gamma$-limits of the two dimensional reduction problems defined by

$$
\frac{1}{\delta} \int_{\omega^{+\delta}} W(D u) d x, \quad \frac{1}{\delta} \int_{\omega^{-\delta}} W(D u) d x
$$

while the third term, arising in the limit from the energy contribution close to the connecting zones, represents the asymptotic memory of the sieve: it is the above mentioned interfacial energy.

The main results of this paper are stated in Theorem 3.3 and Theorem 3.6. In Theorem 3.3 we prove a $\Gamma$-convergence result for the sequence of functionals (1.1) while in Theorem 3.6 we give an explicit characterization of the interfacial energy term occurring in the $\Gamma$-limit. More precisely, for every sequence $\left(\varepsilon_{j}\right)$ converging to zero, we set $\delta_{j}:=\delta\left(\varepsilon_{j}\right), r_{j}:=r\left(\varepsilon_{j}, \delta_{j}\right), \Omega_{j}:=\Omega_{\varepsilon_{j}, r_{j}}^{\delta_{j}}$ and

$$
\mathcal{F}_{j}(u):= \begin{cases}\frac{1}{\delta_{j}} \int_{\Omega_{j}} W(D u) d x & \text { if } u \in W^{1, p}\left(\Omega_{j} ; \mathbb{R}^{m}\right) \\ +\infty & \text { otherwise. }\end{cases}
$$

Up to subsequence we can define

$$
\ell:=\lim _{j \rightarrow+\infty} \frac{r_{j}}{\delta_{j}} \quad \text { and } \quad g(F):=\lim _{j \rightarrow+\infty} r_{j}^{p} \mathcal{Q}_{n} W\left(r_{j}^{-1} F\right) .
$$

where $\mathcal{Q}_{n} W$ is the $n$-quasiconvexification of $W$.

If $\ell \in(0,+\infty]$ and

$$
0<R^{(\ell)}:=\lim _{j \rightarrow+\infty} \frac{r_{j}^{n-1-p}}{\varepsilon_{j}^{n-1}}<+\infty
$$

then $\left(\mathcal{F}_{j}\right) \Gamma$-converges to

$$
\mathcal{F}^{(\ell)}\left(u^{+}, u^{-}\right)=\int_{\omega} \mathcal{Q}_{n-1} \bar{W}\left(D_{\alpha} u^{+}\right) d x_{\alpha}+\int_{\omega} \mathcal{Q}_{n-1} \bar{W}\left(D_{\alpha} u^{-}\right) d x_{\alpha}+R^{(\ell)} \int_{\omega} \varphi^{(\ell)}\left(u^{+}-u^{-}\right) d x_{\alpha}
$$

on $W^{1, p}\left(\omega ; \mathbb{R}^{m}\right) \times W^{1, p}\left(\omega ; \mathbb{R}^{m}\right)$ with respect to the convergence introduced in Definition 3.1 , where $\bar{W}(\bar{F}):=\inf \left\{W(\bar{F} \mid z): z \in \mathbb{R}^{m}\right\}, \mathcal{Q}_{n-1} \bar{W}$ is the $(n-1)$-quasiconvexification of $\bar{W}$ and $\varphi^{(\ell)}: \mathbb{R}^{m} \rightarrow$ $[0,+\infty)$ is a locally Lipschitz continuous function for any $\ell \in[0,+\infty]$. Similarly, if $\ell=0$ and

$$
0<R^{(0)}:=\lim _{j \rightarrow+\infty} \frac{r_{j}^{n-p}}{\delta_{j} \varepsilon_{j}^{n-1}}<+\infty
$$

then we still have $\Gamma$-convergence, as above, to

$$
\mathcal{F}^{(0)}\left(u^{+}, u^{-}\right)=\int_{\omega} \mathcal{Q}_{n-1} \bar{W}\left(D_{\alpha} u^{+}\right) d x_{\alpha}+\int_{\omega} \mathcal{Q}_{n-1} \bar{W}\left(D_{\alpha} u^{-}\right) d x_{\alpha}+R^{(0)} \int_{\omega} \varphi^{(0)}\left(u^{+}-u^{-}\right) d x_{\alpha}
$$

on $W^{1, p}\left(\omega ; \mathbb{R}^{m}\right) \times W^{1, p}\left(\omega ; \mathbb{R}^{m}\right)$.

For any $\ell \in[0,+\infty], \varphi^{(\ell)}$ is described by the following nonlinear capacitary-type formulas: 
(1) if $\ell=+\infty$, then

$$
\begin{array}{r}
\varphi^{(\infty)}(z)=\inf \left\{\int_{\mathbb{R}^{n-1}}\left(\mathcal{Q}_{n-1} \bar{g}\left(D_{\alpha} \zeta^{+}\right)+\mathcal{Q}_{n-1} \bar{g}\left(D_{\alpha} \zeta^{-}\right)\right) d x_{\alpha}: \zeta^{ \pm} \in W_{\text {loc }}^{1, p}\left(\mathbb{R}^{n-1} ; \mathbb{R}^{m}\right)\right. \\
\zeta^{+}=\zeta^{-} \text {in } B_{1}^{n-1}(0), \quad D_{\alpha} \zeta^{ \pm} \in L^{p}\left(\mathbb{R}^{n-1} ; \mathbb{R}^{m \times(n-1)}\right), \\
\left.\left(\zeta^{+}-z\right), \zeta^{-} \in L^{p^{*}}\left(\mathbb{R}^{n-1} ; \mathbb{R}^{m}\right)\right\},
\end{array}
$$

where again, $\bar{g}(\bar{F}):=\inf \left\{g(\bar{F} \mid z): z \in \mathbb{R}^{m}\right\}$ and $\mathcal{Q}_{n-1} \bar{g}$ is the $(n-1)$-quasiconvexification of $\bar{g}$,

(2) if $\ell=0$, then

$$
\begin{array}{r}
\varphi^{(0)}(z)=\inf \left\{\int_{\mathbb{R}^{n} \backslash C_{1, \infty}} g(D \zeta) d x: \zeta \in W_{\mathrm{loc}}^{1, p}\left(\mathbb{R}^{n} \backslash C_{1, \infty} ; \mathbb{R}^{m}\right), D \zeta \in L^{p}\left(\mathbb{R}^{n} \backslash C_{1, \infty} ; \mathbb{R}^{m \times n}\right),\right. \\
\left.\zeta-z \in L^{p}\left(0,+\infty ; L^{p^{*}}\left(\mathbb{R}^{n-1} ; \mathbb{R}^{m}\right)\right) \text { and } \zeta \in L^{p}\left(-\infty, 0 ; L^{p^{*}}\left(\mathbb{R}^{n-1} ; \mathbb{R}^{m}\right)\right)\right\}
\end{array}
$$

(3) if $\ell \in(0,+\infty)$, then

$$
\begin{aligned}
& \varphi^{(\ell)}(z)=\inf \left\{\int_{\mathbb{R}^{n-1} \times(-1,1)} g\left(D_{\alpha} \zeta \mid \ell D_{n} \zeta\right) d x: \zeta \in W_{\mathrm{loc}}^{1, p}\left(\left(\mathbb{R}^{n-1} \times(-1,1)\right) \backslash C_{1, \infty} ; \mathbb{R}^{m}\right),\right. \\
& D \zeta \in L^{p}\left(\mathbb{R}^{n-1} \times(-1,1) ; \mathbb{R}^{m}\right), \quad \zeta-z \in L^{p}\left((0,1) ; L^{p^{*}}\left(\mathbb{R}^{n-1} ; \mathbb{R}^{m}\right)\right) \\
& \left.\zeta \in L^{p}\left((-1,0) ; L^{p^{*}}\left(\mathbb{R}^{n-1} ; \mathbb{R}^{m}\right)\right)\right\}
\end{aligned}
$$

where $C_{1, \infty}:=\left\{\left(x_{\alpha}, 0\right) \in \mathbb{R}^{n}: 1 \leq\left|x_{\alpha}\right|\right\}$.

Before giving a brief heuristic description of each regime, we want to point out that whatever the value of $\ell$ is, the interfacial energy density $\varphi^{(\ell)}$ corresponds to a cohesive interface where the surface energy increases continuously from zero with the jump in the deformation across the interface.

(1) The case $\ell=+\infty$ corresponds to $\delta_{j} \ll r_{j} \ll \varepsilon_{j}$, thus we expect $r_{j}$ to depend only on $\varepsilon_{j}$. In this case we have a separation of scales effect. We first consider $r_{j}$ and $\varepsilon_{j}$ as 'fixed' and let $\delta_{j}$ tend to zero as if we were dealing with two pure dimensional reduction problems stated separately on the upper and lower part (with respect to the sieve plane) of $\Omega_{j}$. Then this first limit procedure yields two functionals being both a copy of the functional in [23]. Since the two corresponding limit deformations $u^{+}$and $u^{-}$must match inside each connecting zone, the above two terms are not completely decoupled. We are then in a situation quite similar to that of $[3,4]$, except that here both periodically 'perforated' $(n-1)$-dimensional bodies are linked each other through the 'perforations'; i.e., through the holes of the sieve and not through the sieve itself. Thus it is coherent to find a critical size of order $\varepsilon^{(n-1) /(n-1-p)}$. Moreover this strong separation between the phenomena of dimension reduction and 'perforation' leads to anisotropy as it can be seen, for instance, also by an inspection of the proof of Lemma 6.2 which shows that the extra interfacial energy term appears thanks to suitable dilatations having a different scaling in the in-plane and transverse variables. Finally we note that the formula for $\varphi^{(\infty)}$ is given in terms of a 'Le Dret-Raoult type' functional involving the limit of the right capacitary scaling (that is, involving the function $g$ ).

(2) The case $\ell=0$ corresponds to $r_{j} \ll \delta_{j} \ll \varepsilon_{j}$. In this case we expect that the critical size $r_{j}$ depends on both $\delta_{j}$ and $\varepsilon_{j}$. Indeed, as already pointed out, $r_{j}$ is of order $\delta_{j}^{1 /(n-p)} \varepsilon_{j}^{(n-1) /(n-p)}$. Note that for $\delta_{j}=\varepsilon_{j}$ we recover $\varepsilon^{n /(n-p)}$ that is the critical size obtained in [2]; moreover $\varphi^{(0)}$ turns out to coincide with the function $\varphi$ in [2] (see Remark 7.13). Contrary to the previous case, now the isotropy is preserved in fact here the dimensional reduction and 'perforation' processes are not completely decoupled: 
the reduction parameter $\delta_{j}$ is forced between both parameters $r_{j}$ and $\varepsilon_{j}$. This can be seen also by noticing that now the scaling leading to the interfacial energy is the same in every direction (see for instance the proof of the $\Gamma$-limsup inequality). Moreover now in $\varphi^{(0)}$ the reduction procedure is not explicit but only witnessed by the boundary conditions expressed only on the lateral part of the boundary of the considered domain.

(3) The case $\ell \in(0,+\infty)$ corresponds to $r_{j} \sim \delta_{j} \ll \varepsilon_{j}$. In this case the separation of scales effect does not take place and the two previous scalings turn out to be equivalent $\left(R^{(0)}=\ell R^{(\infty)}\right)$. Moreover we find that the interfacial energy is continuous with respect to $\ell$ in the extreme regimes; i.e., $R^{(\ell)} \varphi^{(\ell)}(z) \rightarrow$ $R^{(\infty)} \varphi^{(\infty)}(z)$ as $\ell \rightarrow+\infty$ and $R^{(\ell)} \varphi^{(\ell)}(z) \rightarrow R^{(0)} \varphi^{(0)}(z)$ as $\ell \rightarrow 0$. Finally, as in the previous case, the lateral boundary conditions are the only mean describing the dimensional reduction phenomenon in the procedure leading to $\varphi^{(\ell)}$.

This paper is organized as follows: after recalling some useful notation in Section 2, we state the main results, Theorem 3.3 and Theorem 3.6, in Section 3. Then, in Section 4 we list some auxiliary results as rescaled Poincaré type inequalities and joining lemmas. Section 5 is devoted to give a preliminary definition of the interfacial energy density as limit of minimum problems. In Section 6 we prove the $\Gamma$-convergence result (Theorem 3.3). It is only in Section 7 that we compute the explicit expression of the interfacial energy density for each regime (Theorem 3.6).

\section{Notation}

Given $x \in \mathbb{R}^{n}$, we set $x=\left(x_{\alpha}, x_{n}\right)$ where $x_{\alpha}:=\left(x_{1}, \ldots, x_{n-1}\right)$ is the in-plane variable and $D_{\alpha}:=$ $\left(\frac{\partial}{\partial x_{1}}, \ldots, \frac{\partial}{\partial x_{n-1}}\right)$ (resp. $\left.D_{n}\right)$ the derivative with respect to $x_{\alpha}$ (resp. $x_{n}$ ).

The notation $\mathbb{R}^{m \times n}$ stands for the set of $m \times n$ real matrices. Given a matrix $F \in \mathbb{R}^{m \times n}$, we write $F=\left(\bar{F} \mid F_{n}\right)$ where $\bar{F}=\left(F_{1}, \ldots, F_{n-1}\right)$ and $F_{i}$ denotes the $i$-th column of $F, 1 \leq i \leq n$ and $\bar{F} \in \mathbb{R}^{m \times(n-1)}$.

The Lebesgue measure in $\mathbb{R}^{n}$ will be denoted by $\mathcal{L}^{n}$ and the Hausdorff $(n-1)$-dimensional measure by $\mathcal{H}^{n-1}$. Let $A$ be an open subset of $\mathbb{R}^{d}(d=n-1, d=n)$. If $s \in[1,+\infty]$, we use standard notation for Lebesgue and Sobolev spaces $L^{s}\left(A ; \mathbb{R}^{m}\right)$ and $W^{1, s}\left(A ; \mathbb{R}^{m}\right)$.

Let $\omega$ be a bounded open subset of $\mathbb{R}^{n-1}$ and $I=(-1,1)$, we define $\Omega:=\omega \times I$. In the sequel, we will identify $L^{s}\left(\omega ; \mathbb{R}^{m}\right)$ (resp. $\left.W^{1, s}\left(\omega ; \mathbb{R}^{m}\right)\right)$ with the space of functions $v \in L^{s}\left(\Omega ; \mathbb{R}^{m}\right)\left(\operatorname{resp} . W^{1, s}\left(\Omega ; \mathbb{R}^{m}\right)\right)$ such that $D_{n} v=0$ in the sense of distribution.

For every $(a, b) \subset \mathbb{R}$ with $a<b$ and $q_{1}, q_{2} \geq 1, L^{q_{1}}\left(a, b ; L^{q_{2}}\left(\mathbb{R}^{(n-1)} ; \mathbb{R}^{m}\right)\right)$ is the space of measurable $m$-vectorial functions $\zeta$ such that

$$
\int_{b}^{a}\left(\int_{\mathbb{R}^{n-1}}\left|\zeta\left(x_{\alpha}, x_{n}\right)\right|^{q_{2}} d x_{\alpha}\right)^{\frac{q_{1}}{q_{2}}} d x_{n}<+\infty .
$$

Let $a \in \mathbb{R}^{n-1}$ and $\rho>0$, we denote by $B_{\rho}^{n-1}(a)$ the open ball of $\mathbb{R}^{n-1}$ of center $a$ and radius $\rho$ and by $Q_{\rho}^{n-1}(a)$ the open cube of $\mathbb{R}^{n-1}$ with center $a$ and length side $\rho$. We write $B_{\rho}^{n-1}$ instead of $B_{\rho}^{n-1}(0)$ not to overburden notation. Let $x_{i}^{\varepsilon}=i \varepsilon$ with $i \in \mathbb{Z}^{n-1}$, we set $Q_{i, \varepsilon}^{n-1}:=Q_{\varepsilon}^{n-1}\left(x_{i}^{\varepsilon}\right)$.

We define $U^{+a}:=U \times(0, a)$ and $U^{-a}:=U \times(-a, 0)$ with $U \subseteq \mathbb{R}^{n-1}$ and $a>0$, while if $a=1$, then $U^{+}=U^{+1}$ and $U^{-}=U^{-1}$.

We set $C_{1, \infty}:=\left\{\left(x_{\alpha}, 0\right) \in \mathbb{R}^{n}: 1 \leq\left|x_{\alpha}\right|\right\}$ and $C_{1, N}:=\left\{\left(x_{\alpha}, 0\right) \in \mathbb{R}^{n}: 1 \leq\left|x_{\alpha}\right|<N\right\}$ for every $N>1$.

Let $p \geq 1$, we denote by $\operatorname{Cap}_{p}\left(B_{1}^{n-1} ; A\right)$ the $p$-capacity of $B_{1}^{n-1}$ with respect to $A \subseteq \mathbb{R}^{d}$ :

$$
\operatorname{Cap}_{p}\left(B_{1}^{n-1} ; A\right)=\inf \left\{\int_{A}|D \psi|^{p} d x: \psi \in W_{0}^{1, p}(A) \text { and } \psi=1 \text { on } B_{1}^{n-1}\right\} .
$$

The letter $c$ will stand for a generic strictly-positive constant which may vary from line to line and expression to expression within the same formula.

\section{Statements of the Main Results}

Since we are going to work with varying domains, we have to precise the meaning of 'converging sequences'. 
Definition 3.1. Let $\Omega_{j}=\omega^{+\delta_{j}} \cup \omega^{-\delta_{j}} \cup\left(\omega_{r_{j}, \varepsilon_{j}} \times\{0\}\right)$. Given a sequence $\left(u_{j}\right) \subset W^{1, p}\left(\Omega_{j} ; \mathbb{R}^{m}\right)$, we define $\hat{u}_{j}\left(x_{\alpha}, x_{n}\right):=u_{j}\left(x_{\alpha}, \delta_{j} x_{n}\right)$. We say that $\left(u_{j}\right)$ converges (resp. converges weakly) to $\left(u^{+}, u^{-}\right) \in$ $W^{1, p}\left(\omega ; \mathbb{R}^{m}\right) \times W^{1, p}\left(\omega ; \mathbb{R}^{m}\right)$ if we have

$$
\begin{array}{llll}
\hat{u}_{j}^{+}:=\left.\hat{u}_{j}\right|_{\omega^{+}} \rightarrow u^{+} \text {in } & L^{p}\left(\omega^{+} ; \mathbb{R}^{m}\right) & \left(\text { resp. weakly in } W^{1, p}\left(\omega^{+} ; \mathbb{R}^{m}\right)\right), \\
\hat{u}_{j}^{-}:=\left.\hat{u}_{j}\right|_{\omega^{-}} \rightarrow u^{-} \text {in } & L^{p}\left(\omega^{-} ; \mathbb{R}^{m}\right) & \text { (resp. weakly in } \left.W^{1, p}\left(\omega^{-} ; \mathbb{R}^{m}\right)\right) .
\end{array}
$$

Similarly if we replace $\Omega_{j}$ by $\omega^{ \pm \delta_{j}}$.

We say that the sequence $\left(\left|D u_{j}\right|^{p} / \delta_{j}\right)$ is equi-integrable on $\omega^{ \pm \delta_{j}}$ if $\left(\left|\left(D_{\alpha} \hat{u}_{j} \mid \frac{1}{\delta_{j}} D_{n} \hat{u}_{j}\right)\right|^{p}\right)$ is equiintegrable on $\omega^{ \pm}$.

Remark 3.2. By virtue of Definition 3.1, a sequence $\left(u_{j}\right) \subset W^{1, p}\left(\Omega_{j} ; \mathbb{R}^{m}\right)$ converges to $\left(u^{+}, u^{-}\right) \in$ $W^{1, p}\left(\omega ; \mathbb{R}^{m}\right) \times W^{1, p}\left(\omega ; \mathbb{R}^{m}\right)$ if and only if

$$
\lim _{j \rightarrow+\infty} \frac{1}{\delta_{j}} \int_{\omega^{ \pm \delta_{j}}}\left|u_{j}-u^{ \pm}\right|^{p} d x=0
$$

while (3.1) and

$$
\sup _{j \in \mathbb{N}} \frac{1}{\delta_{j}} \int_{\omega^{ \pm \delta_{j}}}\left|D u_{j}\right|^{p} d x=\sup _{j \in \mathbb{N}} \int_{\omega^{ \pm}}\left|\left(D_{\alpha} \hat{u}_{j} \mid \frac{1}{\delta_{j}} D_{n} \hat{u}_{j}\right)\right|^{p} d x<+\infty
$$

imply the weak convergence.

Note that Remark 3.2 is still valid if we consider the domain $\omega^{+\delta_{j}} \cup \omega^{-\delta_{j}}$ in place of $\Omega_{j}$.

The main results of this paper are the following:

Theorem 3.3 ( $\Gamma$-convergence). Let $1<p<n-1$. Let $\omega$ be a bounded open subset of $\mathbb{R}^{n-1}$ satisfying $\mathcal{H}^{n-1}(\partial \omega)=0$ and $W: \mathbb{R}^{m \times n} \rightarrow[0,+\infty)$ be a Borel function such that $W(0)=0$ and satisfying a growth condition of order $p$ : there exists a constant $\beta>0$ such that

$$
|F|^{p}-1 \leq W(F) \leq \beta\left(|F|^{p}+1\right), \quad \text { for every } F \in \mathbb{R}^{m \times n} .
$$

Let $\left(\varepsilon_{j}\right),\left(\delta_{j}\right)$ and $\left(r_{j}\right)$ be sequences of strictly positive numbers converging to zero such that

$$
\lim _{j \rightarrow+\infty} \frac{\delta_{j}}{\varepsilon_{j}}=0
$$

and set

$$
\ell:=\lim _{j \rightarrow+\infty} \frac{r_{j}}{\delta_{j}} .
$$

If

or

$$
\ell \in(0,+\infty], \quad \text { and } \quad 0<R^{(\ell)}:=\lim _{j \rightarrow+\infty} \frac{r_{j}^{n-1-p}}{\varepsilon_{j}^{n-1}}<+\infty
$$

$$
\ell=0, \quad \text { and } \quad 0<R^{(0)}:=\lim _{j \rightarrow+\infty} \frac{r_{j}^{n-p}}{\delta_{j} \varepsilon_{j}^{n-1}}<+\infty,
$$

then, up to an extraction, the sequence of functionals $\mathcal{F}_{j}: L^{p}\left(\Omega_{j} ; \mathbb{R}^{m}\right) \rightarrow[0,+\infty]$ defined by

$$
\mathcal{F}_{j}(u):= \begin{cases}\frac{1}{\delta_{j}} \int_{\Omega_{j}} W(D u) d x & \text { if } u \in W^{1, p}\left(\Omega_{j} ; \mathbb{R}^{m}\right), \\ +\infty & \text { otherwise }\end{cases}
$$

$\Gamma$-converges to

$$
\mathcal{F}^{(\ell)}\left(u^{+}, u^{-}\right)=\int_{\omega} \mathcal{Q}_{n-1} \bar{W}\left(D_{\alpha} u^{+}\right) d x_{\alpha}+\int_{\omega} \mathcal{Q}_{n-1} \bar{W}\left(D_{\alpha} u^{-}\right) d x_{\alpha}+R^{(\ell)} \int_{\omega} \varphi^{(\ell)}\left(u^{+}-u^{-}\right) d x_{\alpha}
$$

on $W^{1, p}\left(\omega ; \mathbb{R}^{m}\right) \times W^{1, p}\left(\omega ; \mathbb{R}^{m}\right)$ with respect to the convergence introduced in Definition 3.1 , where $\bar{W}(\bar{F}):=$ $\inf \left\{W(\bar{F} \mid z): z \in \mathbb{R}^{m}\right\}, \mathcal{Q}_{n-1} \bar{W}$ is the $(n-1)$-quasiconvexification of $\bar{W}$ and $\varphi^{(\ell)}: \mathbb{R}^{m} \rightarrow[0,+\infty)$ is a locally Lipschitz continuous function for any $\ell \in[0,+\infty]$. 
Remark 3.4. Note that if $\ell \in(0,+\infty]$ the only meaningful scaling for $r_{j}$ is that of order $\varepsilon_{j}^{(n-1) /(n-1-p)}$; i.e., for both $R^{(\ell)}=0$ and $R^{(\ell)}=+\infty$ we loose the asymptotic memory of the sieve. In fact, if $R^{(\ell)}=0$, we obtain two uncoupled problems in the limit, while if $R^{(\ell)}=+\infty$, limit deformations $\left(u^{+}, u^{-}\right)$with finite energy are continuous across the mid-section $\left(u^{+}=u^{-}\right.$in $\left.\omega\right)$ as in Le Dret-Raoult [23]. Similarly, for $\ell=0$.

Remark 3.5. If $\ell \in(0,+\infty)$ then

$$
0<R^{(\ell)}=\lim _{j \rightarrow+\infty} \frac{r_{j}^{n-1-p}}{\varepsilon_{j}^{n-1}}<+\infty \quad \text { if and only if } \quad 0<R^{(0)}=\lim _{j \rightarrow+\infty} \frac{r_{j}^{n-p}}{\delta_{j} \varepsilon_{j}^{n-1}}<+\infty ;
$$

hence, in this case the two meaningful scalings are equivalent.

The following result provides a characterization of the interfacial energy density $\varphi^{(\ell)}$ for each $\ell \in[0,+\infty]$.

Theorem 3.6 (Representation formulas). Let $p^{*}=(n-1) p /(n-1-p)$ be the Sobolev exponent in dimension $(n-1)$. Then, upon extracting a subsequence, there exists the limit

$$
g(F):=\lim _{j \rightarrow+\infty} r_{j}^{p} \mathcal{Q}_{n} W\left(r_{j}^{-1} F\right)
$$

for all $F \in \mathbb{R}^{m \times n}$, where $\mathcal{Q}_{n} W$ denotes the $n$-quasiconvexification of $W$, so that:

if $\ell \in(0,+\infty)$,

$$
\begin{array}{r}
\varphi^{(\ell)}(z):=\inf \left\{\int_{\left(\mathbb{R}^{n-1} \times I\right) \backslash C_{1, \infty}} g\left(D_{\alpha} \zeta \mid \ell D_{n} \zeta\right) d x: \zeta \in W_{\mathrm{loc}}^{1, p}\left(\left(\mathbb{R}^{n-1} \times I\right) \backslash C_{1, \infty} ; \mathbb{R}^{m}\right),\right. \\
D \zeta \in L^{p}\left(\left(\mathbb{R}^{n-1} \times I\right) \backslash C_{1, \infty} ; \mathbb{R}^{m \times n}\right), \quad \zeta-z \in L^{p}\left(0,1 ; L^{p^{*}}\left(\mathbb{R}^{n-1} ; \mathbb{R}^{m}\right)\right) \\
\left.\quad \zeta \in L^{p}\left(-1,0 ; L^{p^{*}}\left(\mathbb{R}^{n-1} ; \mathbb{R}^{m}\right)\right)\right\} ;
\end{array}
$$

if $\ell=+\infty$

$$
\begin{array}{r}
\varphi^{(\infty)}(z):=\inf \left\{\int_{\mathbb{R}^{n-1}}\left(\mathcal{Q}_{n-1} \bar{g}\left(D_{\alpha} \zeta^{+}\right)+\mathcal{Q}_{n-1} \bar{g}\left(D_{\alpha} \zeta^{-}\right)\right) d x_{\alpha}: \zeta^{ \pm} \in W_{\mathrm{loc}}^{1, p}\left(\mathbb{R}^{n-1} ; \mathbb{R}^{m}\right),\right. \\
\zeta^{+}=\zeta^{-} \text {in } B_{1}^{n-1}, \quad D_{\alpha} \zeta^{ \pm} \in L^{p}\left(\mathbb{R}^{n-1} ; \mathbb{R}^{m \times(n-1)}\right), \\
\left.\left(\zeta^{+}-z\right), \zeta^{-} \in L^{p^{*}}\left(\mathbb{R}^{n-1} ; \mathbb{R}^{m}\right)\right\},
\end{array}
$$

where $\bar{g}(\bar{F}):=\inf \left\{g(\bar{F} \mid z): z \in \mathbb{R}^{m}\right\}$ and $\mathcal{Q}_{n-1} \bar{g}$ is the $(n-1)$-quasiconvexification of $\bar{g}$; if $\ell=0$

$$
\begin{array}{r}
\varphi^{(0)}(z)=\inf \left\{\int_{\mathbb{R}^{n} \backslash C_{1, \infty}} g(D \zeta) d x: \zeta \in W_{\mathrm{loc}}^{1, p}\left(\mathbb{R}^{n} \backslash C_{1, \infty} ; \mathbb{R}^{m}\right), D \zeta \in L^{p}\left(\mathbb{R}^{n} \backslash C_{1, \infty} ; \mathbb{R}^{m \times n}\right),\right. \\
\left.\zeta-z \in L^{p}\left(0,+\infty ; L^{p^{*}}\left(\mathbb{R}^{n-1} ; \mathbb{R}^{m}\right)\right), \zeta \in L^{p}\left(-\infty, 0 ; L^{p^{*}}\left(\mathbb{R}^{n-1} ; \mathbb{R}^{m}\right)\right)\right\},
\end{array}
$$

for all $z \in \mathbb{R}^{m}$.

Remark 3.7. Without loss of generality we may assume that $W$ is quasiconvex (upon first relaxing the energy); hence, by (3.3), $W$ satisfies the following $p$-Lipschitz condition (see e.g. [19]):

$$
\left|W\left(F_{1}\right)-W\left(F_{2}\right)\right| \leq c\left(1+\left|F_{1}\right|^{p-1}+\left|F_{2}\right|^{p-1}\right)\left|F_{1}-F_{2}\right|, \quad \text { for all } F_{1}, F_{2} \in \mathbb{R}^{m \times n} .
$$




\section{Preliminary Results}

4.1. Some rescaled Poincaré Inequalities. Since we deal with varying domains, depending on different parameters, it is useful to note how the constant in Poincaré type inequalities rescale with respect to such parameters.

Lemma 4.1. Let $A$ be an open bounded and connected subset of $\mathbb{R}^{n-1}$ with Lipschitz boundary and let $A_{\rho}:=\rho A$ for $\rho>0$.

(i) There exists a constant $c>0$ (depending only on $(A, n, p)$ ) such that for every $\rho, \delta>0$

$$
\int_{A_{\rho}^{ \pm \delta}}\left|u-\bar{u}_{A_{\rho}^{ \pm \delta}}\right|^{p} d x \leq c \int_{A_{\rho}^{ \pm \delta}}\left(\rho^{p}\left|D_{\alpha} u\right|^{p}+\delta^{p}\left|D_{n} u\right|^{p}\right) d x
$$

for every $u \in W^{1, p}\left(A_{\rho}^{ \pm \delta} ; \mathbb{R}^{m}\right)$ where $\bar{u}_{A_{\rho}^{ \pm \delta}}=f_{A_{\rho}^{ \pm \delta}} u d x$.

(ii) If $B$ is an open and connected subset of $A$ with Lipschitz boundary and $B_{\rho}:=\rho B$ then there exists a constant $c>0$ (depending only on $(A, B, n, p)$ ) such that for every $\rho, \delta>0$

$$
\int_{A_{\rho}^{ \pm \delta}}\left|u-\bar{u}_{B_{\rho}^{ \pm \delta}}\right|^{p} d x \leq c \int_{A_{\rho}^{ \pm \delta}}\left(\rho^{p}\left|D_{\alpha} u\right|^{p}+\delta^{p}\left|D_{n} u\right|^{p}\right) d x
$$

for every $u \in W^{1, p}\left(A_{\rho}^{ \pm \delta} ; \mathbb{R}^{m}\right)$ where $\bar{u}_{B_{\rho}^{ \pm \delta}}=f_{B_{\rho}^{ \pm \delta}} u d x$.

Proof. Let us define $v\left(x_{\alpha}, x_{n}\right):=u\left(\rho x_{\alpha}, \delta x_{n}\right)$ then $v \in W^{1, p}\left(A^{ \pm} ; \mathbb{R}^{m}\right)$. By a change of variable, we get that $\bar{u}_{A_{\rho}^{ \pm \delta}}=\bar{v}_{A^{ \pm}}$. Moreover, by the Poincaré Inequality, there exists a constant $c=c(A, n, p)>0$ such that

$$
\begin{aligned}
\int_{A_{\rho}^{ \pm \delta}}\left|u-\bar{u}_{A_{\rho}^{ \pm \delta}}\right|^{p} d x & =\delta \rho^{n-1} \int_{A^{ \pm}}\left|v-\bar{v}_{A^{ \pm}}\right|^{p} d y \\
& \leq c \delta \rho^{n-1} \int_{A^{ \pm}}|D v|^{p} d y \\
& =c \int_{A_{\rho}^{ \pm \delta}}\left(\rho^{p}\left|D_{\alpha} u\right|^{p}+\delta^{p}\left|D_{n} u\right|^{p}\right) d x
\end{aligned}
$$

and it completes the proof of (i). Now, if $B_{\rho} \subset A_{\rho}$, we get that

$$
\begin{aligned}
& \int_{A_{\rho}^{ \pm \delta}}\left|u-\bar{u}_{B_{\rho}^{ \pm \delta}}\right|^{p} d x \\
\leq & c\left(\int_{A_{\rho}^{ \pm \delta}}\left|u-\bar{u}_{A_{\rho}^{ \pm \delta}}\right|^{p} d x+\delta \rho^{n-1} \mathcal{H}^{n-1}(A)\left|\bar{u}_{A_{\rho}^{ \pm \delta}}-\bar{u}_{B_{\rho}^{ \pm \delta}}\right|^{p}\right) \\
\leq & c \int_{A_{\rho}^{ \pm \delta}}\left|u-\bar{u}_{A_{\rho}^{ \pm \delta}}\right|^{p} d x+c \frac{\mathcal{H}^{n-1}(A)}{\mathcal{H}^{n-1}(B)}\left(\int_{B_{\rho}^{ \pm \delta}}\left|u-\bar{u}_{A_{\rho}^{ \pm \delta}}\right|^{p} d x+\int_{B_{\rho}^{ \pm \delta}}\left|u-\bar{u}_{B_{\rho}^{ \pm \delta}}\right|^{p} d x\right) \\
\leq & c \int_{A_{\rho}^{ \pm \delta}}\left(\rho^{p}\left|D_{\alpha} u\right|^{p}+\delta^{p}\left|D_{n} u\right|^{p}\right) d x .
\end{aligned}
$$

4.2. A joining lemma on varying domains. If not otherwise specified, in all what follows the convergence of a sequence of functions has to be intended in the sense of Definition 3.1.

The following lemma, is the key tool in the proof of Theorem 3.3. It is a technical result which allows to modify sequences of functions 'near' the sets $B_{r_{j}}^{(n-1)}\left(x_{i}^{\varepsilon_{j}}\right)$. It is very close in spirit to Lemma 3.4 in [2] although now the geometry of the problem yields a different construction involving suitable cylindrical (instead of spherical) annuli to surround the connecting zones. 
Lemma 4.2. Let $\left(\varepsilon_{j}\right),\left(\delta_{j}\right)$ be sequences of strictly positive numbers converging to 0 and such that $\delta_{j} \ll \varepsilon_{j}$. Let $\left(u_{j}\right) \subset W^{1, p}\left(\omega^{+\delta_{j}} \cup \omega^{-\delta_{j}} ; \mathbb{R}^{m}\right)$ be a sequence converging to $\left(u^{+}, u^{-}\right) \in W^{1, p}\left(\omega ; \mathbb{R}^{m}\right) \times W^{1, p}\left(\omega ; \mathbb{R}^{m}\right)$ satisfying $\sup _{j} \mathcal{F}_{j}\left(u_{j}\right)<+\infty$; let $k \in \mathbb{N}$. Set $\rho_{j}=\gamma \varepsilon_{j}$ with $\gamma<1 / 2$ and

$$
Z_{j}:=\left\{i \in \mathbb{Z}^{n-1}: \operatorname{dist}\left(x_{i}^{\varepsilon_{j}}, \mathbb{R}^{n-1} \backslash \omega\right)>\varepsilon_{j}\right\} .
$$

For every $i \in Z_{j}$, there exists $k_{i} \in\{0, \ldots, k-1\}$ such that having set

$$
\begin{gathered}
C_{j}^{i}:=\left\{x_{\alpha} \in \omega: 2^{-k_{i}-1} \rho_{j}<\left|x_{\alpha}-x_{i}^{\varepsilon_{j}}\right|<2^{-k_{i}} \rho_{j}\right\}, \\
u_{j}^{i \pm}:=f_{\left(C_{j}^{i}\right)^{ \pm \delta_{j}}} u_{j} d x
\end{gathered}
$$

and

$$
\rho_{j}^{i}:=\frac{3}{4} 2^{-k_{i}} \rho_{j},
$$

there exists a sequence $\left(w_{j}\right) \subset W^{1, p}\left(\omega^{+\delta_{j}} \cup \omega^{-\delta_{j}} ; \mathbb{R}^{m}\right)$ weakly converging to $\left(u^{+}, u^{-}\right)$such that

$$
\begin{gathered}
w_{j}=u_{j} \quad \text { in }\left(\omega \backslash \bigcup_{i \in Z_{j}} C_{j}^{i}\right)^{ \pm \delta_{j}}, \\
w_{j}=u_{j}^{i \pm} \text { on }\left(\partial B_{\rho_{j}^{i}}^{n-1}\left(x_{i}^{\varepsilon_{j}}\right)\right)^{ \pm \delta_{j}}
\end{gathered}
$$

and satisfying

$$
\limsup _{j \rightarrow+\infty} \frac{1}{\delta_{j}} \int_{\omega^{ \pm \delta_{j}}}\left|W\left(D w_{j}\right)-W\left(D u_{j}\right)\right| d x \leq \frac{c}{k} .
$$

Proof. For every $j \in \mathbb{N}, i \in Z_{j}, k \in \mathbb{N}$ and $h \in\{0, \ldots, k-1\}$, we define

$$
\begin{gathered}
C_{j}^{i, h}:=\left\{x_{\alpha} \in \omega: 2^{-h-1} \rho_{j}<\left|x_{\alpha}-x_{i}^{\varepsilon_{j}}\right|<2^{-h} \rho_{j}\right\}, \\
\left(u_{j}^{i, h}\right)^{ \pm}:=f_{\left(C_{j}^{i, h}\right)^{ \pm \delta_{j}}} u_{j} d x
\end{gathered}
$$

and

$$
\rho_{j}^{i, h}:=\frac{3}{4} 2^{-h} \rho_{j} .
$$

Let $\phi \equiv \phi_{j}^{i, h} \in \mathcal{C}_{c}^{\infty}\left(C_{j}^{i, h} ;[0,1]\right)$ be a cut-off function such that $\phi=1$ on $\partial B_{\rho_{j}^{i, h}}^{n-1}\left(x_{i}^{\varepsilon_{j}}\right)$ and $\left|D_{\alpha} \phi\right| \leq c / \rho_{j}^{i, h}$. In $\left(C_{j}^{i, h}\right)^{ \pm \delta_{j}}$, we set

$$
w_{j}^{i, h}(x):=\phi\left(x_{\alpha}\right)\left(u_{j}^{i, h}\right)^{ \pm}+\left(1-\phi\left(x_{\alpha}\right)\right) u_{j},
$$

then

$$
\begin{aligned}
\int_{\left(C_{j}^{i, h}\right)^{ \pm \delta_{j}}}\left|D w_{j}^{i, h}\right|^{p} d x & \leq c \int_{\left(C_{j}^{i, h}\right)^{ \pm \delta_{j}}}\left(\left|D_{\alpha} \phi\right|^{p}\left|u_{j}-\left(u_{j}^{i, h}\right)^{ \pm}\right|^{p}+\left|D u_{j}\right|^{p}\right) d x \\
& \leq c \int_{\left(C_{j}^{i, h}\right)^{ \pm \delta_{j}}}\left(\frac{\left|u_{j}-\left(u_{j}^{i, h}\right)^{ \pm}\right|^{p}}{\left(\rho_{j}^{i, h}\right)^{p}}+\left|D u_{j}\right|^{p}\right) d x .
\end{aligned}
$$

Applying Lemma 4.1 (i), with $\rho=\rho_{j}^{i, h}$ and $A_{\rho}=C_{j}^{i, h}$, we have that

$$
\begin{aligned}
& \int_{\left(C_{j}^{i, h}\right)^{ \pm \delta_{j}}}\left|D w_{j}^{i, h}\right|^{p} d x \\
\leq & c \int_{\left(C_{j}^{i, h}\right)^{ \pm \delta_{j}}}\left(\left|D_{\alpha} u_{j}\right|^{p}+\left(\frac{\delta_{j}}{\rho_{j}^{i, h}}\right)^{p}\left|D_{n} u_{j}\right|^{p}\right) d x+c \int_{\left(C_{j}^{i, h}\right)^{ \pm \delta_{j}}}\left|D u_{j}\right|^{p} d x \\
\leq & m_{j}(k, \gamma) c \int_{\left(C_{j}^{i, h}\right)^{ \pm \delta_{j}}}\left|D u_{j}\right|^{p} d x,
\end{aligned}
$$




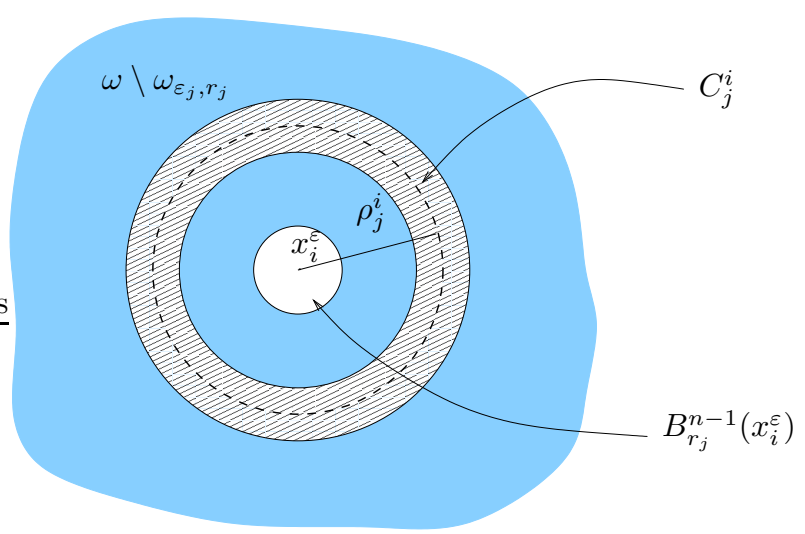

Figure 2. The $(n-1)$-dimensional annuli $C_{j}^{i}$.

where by (4.5)

$$
m_{j}(k, \gamma):=\max \left\{1,\left(\frac{2^{k+1}}{3 \gamma}\right)^{p}\left(\frac{\delta_{j}}{\varepsilon_{j}}\right)^{p}\right\}
$$

and since $\delta_{j} \ll \varepsilon_{j}, m_{j}(k, \gamma) \rightarrow 1$ as $j \rightarrow+\infty$. As

$$
\sum_{h=0}^{k-1} \int_{\left(C_{j}^{i, h}\right)^{ \pm \delta_{j}}}\left(1+\left|D u_{j}\right|^{p}\right) d x \leq \int_{B_{\rho_{j}}^{n-1}\left(x_{i}^{\varepsilon_{j}}\right)^{ \pm \delta_{j}}}\left(1+\left|D u_{j}\right|\right)^{p} d x
$$

there exists $k_{i} \in\{0, \ldots, k-1\}$ such that, having set $C_{j}^{i}:=C_{j}^{i, k_{i}}$, we get

$$
\int_{\left(C_{j}^{i}\right)^{ \pm \delta_{j}}}\left(1+\left|D u_{j}\right|^{p}\right) d x \leq \frac{1}{k} \int_{B_{\rho_{j}}^{n-1}\left(x_{i}^{\varepsilon_{j}}\right)^{ \pm \delta_{j}}}\left(1+\left|D u_{j}\right|^{p}\right) d x .
$$

Hence, if we define the sequence

$$
w_{j}:= \begin{cases}w_{j}^{i, k_{i}} & \text { in }\left(C_{j}^{i}\right)^{ \pm \delta_{j}} \text { for } i \in Z_{j} \\ u_{j} & \text { otherwise }\end{cases}
$$

by the $p$-growth condition (3.3), (4.6), (4.7) and Remark 3.2 we have

$$
\begin{aligned}
\frac{1}{\delta_{j}} \int_{\omega^{ \pm \delta_{j}}}\left|W\left(D w_{j}\right)-W\left(D u_{j}\right)\right| d x & =\sum_{i \in Z_{j}} \frac{1}{\delta_{j}} \int_{\left(C_{j}^{i}\right)^{ \pm \delta_{j}}}\left|W\left(D w_{j}^{i, k_{i}}\right)-W\left(D u_{j}\right)\right| d x \\
& \leq \frac{c}{k} m_{j}(k, \gamma) \sum_{i \in Z_{j}} \frac{1}{\delta_{j}} \int_{B_{\rho_{j}}^{n-1}\left(x_{i}^{\varepsilon_{j}}\right)^{ \pm \delta_{j}}}\left(1+\left|D u_{j}\right|^{p}\right) d x \\
& \leq \frac{c}{k} m_{j}(k, \gamma)\left(1+\sup _{j \in \mathbb{N}} \frac{1}{\delta_{j}} \int_{\omega^{ \pm \delta_{j}}}\left|D u_{j}\right|^{p} d x\right) \\
& \leq \frac{c}{k} m_{j}^{1}(k, \gamma),
\end{aligned}
$$


which concludes the proof of (4.4). Note that, by construction, $\left(w_{j}\right)$ satisfies (4.2) and (4.3) and it converges weakly to $\left(u^{+}, u^{-}\right)$. In fact,

$$
\begin{aligned}
\frac{1}{\delta_{j}} \int_{\omega^{ \pm \delta_{j}}}\left|w_{j}-u^{ \pm}\right|^{p} d x= & \frac{1}{\delta_{j}} \sum_{i \in Z_{j}} \int_{\left(C_{j}^{i}\right)^{ \pm \delta_{j}}}\left|\phi u_{j}^{i \pm}+(1-\phi) u_{j}-u^{ \pm}\right|^{p} d x \\
& +\frac{1}{\delta_{j}} \int_{\omega^{ \pm \delta_{j}} \backslash \bigcup_{i \in Z_{j}}\left(C_{j}^{i}\right)^{ \pm \delta_{j}}}\left|u_{j}-u^{ \pm}\right|^{p} d x \\
\leq & \frac{c}{\delta_{j}} \int_{\omega^{ \pm \delta_{j}}}\left|u_{j}-u^{ \pm}\right|^{p} d x+\frac{c}{\delta_{j}} \sum_{i \in Z_{j}} \int_{\left(C_{j}^{i}\right)^{ \pm \delta_{j}}}\left|u_{j}-u_{j}^{i \pm}\right|^{p} d x
\end{aligned}
$$

while by Lemma 4.1 (i) applied with $\rho=\rho_{j}^{i}$ and since $\delta_{j} \ll \varepsilon_{j}, \rho_{j}^{i} \leq \varepsilon_{j}$, we get

$$
\frac{1}{\delta_{j}} \int_{\omega^{ \pm \delta_{j}}}\left|w_{j}-u^{ \pm}\right|^{p} d x \leq \frac{c}{\delta_{j}} \int_{\omega^{ \pm \delta_{j}}}\left|u_{j}-u^{ \pm}\right|^{p} d x+c \varepsilon_{j}^{p} \frac{1}{\delta_{j}} \int_{\omega^{ \pm \delta_{j}}}\left|D u_{j}\right|^{p} d x .
$$

Moreover by (4.6) we have

$$
\frac{1}{\delta_{j}} \int_{\omega^{ \pm \delta_{j}}}\left|D w_{j}\right|^{p} d x \leq \frac{c}{\delta_{j}} \int_{\omega^{ \pm \delta_{j}}}\left|D u_{j}\right|^{p} d x .
$$

Hence (4.8), (4.9), the convergence of $\left(u_{j}\right)$ towards $\left(u^{+}, u^{-}\right), \sup _{j} \frac{1}{\delta_{j}} \int_{\omega^{ \pm \delta_{j}}}\left|D u_{j}\right|^{p} d x<+\infty$ together with Remark 3.2 imply the weak convergence of $\left(w_{j}\right)$ towards $\left(u^{+}, u^{-}\right)$.

Remark 4.3. Note that to prove Lemma 4.2 we essentially use that $\rho_{j}<\varepsilon_{j} / 2$ (but not necessarily equal to $\left.\gamma \varepsilon_{j}\right)$ and $\lim _{j \rightarrow+\infty}\left(\delta_{j} / \rho_{j}\right)=0$. Hence, Lemma 4.2 is still true if we replace the assumptions $\delta_{j} \ll \varepsilon_{j}$ and $\rho_{j}=\gamma \varepsilon_{j}$ by $\rho_{j}<\varepsilon_{j} / 2$ and $\lim _{j \rightarrow+\infty}\left(\delta_{j} / \rho_{j}\right)=0$.

Since we will apply Lemma 4.2 when $\rho_{j}=\gamma \varepsilon_{j}(\gamma<1 / 2)$ and $\delta_{j} \ll \varepsilon_{j}$, we prefer to prove it directly under these assumptions.

If the sequence $\left(\left|D u_{j}\right|^{p} / \delta_{j}\right)$ is equi-integrable on $\omega^{ \pm \delta_{j}}$ (see Definition 3.1), then we do not have to choose for every $i \in Z_{j}$ a suitable annulus $C_{j}^{i}$ but we may consider the same radius independently of $i$ as the following lemma shows.

Lemma 4.4. Let $\left(u_{j}\right),\left(\varepsilon_{j}\right),\left(\delta_{j}\right),\left(\rho_{j}\right)$ and $Z_{j}$ be as in Lemma 4.2 and suppose that $\left(\left|D u_{j}\right|^{p} / \delta_{j}\right)$ is equi-integrable on $\omega^{ \pm \delta_{j}}$. Set

$$
C_{j}^{i}:=\left\{x_{\alpha} \in \omega: \frac{2}{3} \rho_{j}<\left|x_{\alpha}-x_{i}^{\varepsilon_{j}}\right|<\frac{4}{3} \rho_{j}\right\} \quad \text { and } \quad u_{j}^{i \pm}:=f_{\left(C_{j}^{i}\right)^{ \pm \delta_{j}}} u_{j} d x
$$

for every $i \in Z_{j}$. Then, there exists a sequence $\left(w_{j}\right) \subset W^{1, p}\left(\omega^{+\delta_{j}} \cup \omega^{-\delta_{j}} ; \mathbb{R}^{m}\right)$ weakly converging to $\left(u^{+}, u^{-}\right)$such that

$$
\begin{gathered}
w_{j}=u_{j} \quad \text { in }\left(\omega \backslash \bigcup_{i \in Z_{j}} C_{j}^{i}\right)^{ \pm \delta_{j}}, \\
w_{j}=u_{j}^{i \pm} \text { on }\left(\partial B_{\rho_{j}}^{n-1}\left(x_{i}^{\varepsilon_{j}}\right)\right)^{ \pm \delta_{j}}
\end{gathered}
$$

and

$$
\limsup _{j \rightarrow+\infty} \frac{1}{\delta_{j}} \int_{\omega^{ \pm \delta_{j}}}\left|W\left(D w_{j}\right)-W\left(D u_{j}\right)\right| d x \leq o(1) \quad \text { as } \quad \gamma \rightarrow 0^{+} .
$$

Moreover, the sequence $\left(\left|D w_{j}\right|^{p} / \delta_{j}\right)$ is equi-integrable on $\omega^{ \pm \delta_{j}}$.

Proof. Let $\phi \equiv \phi_{j}^{i} \in \mathcal{C}_{c}^{\infty}\left(C_{j}^{i} ;[0,1]\right)$ be a cut-off function such that $\phi=1$ on $\partial B_{\rho_{j}}^{n-1}\left(x_{i}^{\varepsilon_{j}}\right)$ and $\left|D_{\alpha} \phi\right| \leq c / \rho_{j}$. In $\left(C_{j}^{i}\right)^{ \pm \delta_{j}}$, we define

$$
w_{j}^{i}:=\phi\left(x_{\alpha}\right) u_{j}^{i \pm}+\left(1-\phi\left(x_{\alpha}\right)\right) u_{j} .
$$


Then, reasoning as in the proof of Lemma 4.2, we have that

Hence, if we define

$$
\int_{\left(C_{j}^{i}\right)^{ \pm \delta_{j}}} W\left(D w_{j}^{i}\right) d x \leq c \int_{\left(C_{j}^{i}\right)^{ \pm \delta_{j}}}\left(1+\left|D u_{j}\right|^{p}\right) d x .
$$

$$
w_{j}:= \begin{cases}w_{j}^{i} & \text { in }\left(C_{j}^{i}\right)^{ \pm \delta_{j}} \text { for } i \in Z_{j} \\ u_{j} & \text { otherwise }\end{cases}
$$

$w_{j}$ satisfies (4.10) and (4.11). Moreover,

$$
\begin{aligned}
\frac{1}{\delta_{j}} \int_{\omega^{ \pm \delta_{j}}}\left|W\left(D w_{j}\right)-W\left(D u_{j}\right)\right| d x & \leq \sum_{i \in Z_{j}} \frac{1}{\delta_{j}} \int_{\left(C_{j}^{i}\right)^{ \pm \delta_{j}}}\left|W\left(D w_{j}^{i}\right)-W\left(D u_{j}\right)\right| d x \\
& \leq c \sum_{i \in Z_{j}} \frac{1}{\delta_{j}} \int_{\left(B_{4 \rho_{j} / 3}^{n-1}\left(x_{i}^{\varepsilon_{j}}\right) \cap \omega\right)^{ \pm \delta_{j}}}\left(1+\left|D u_{j}\right|^{p}\right) d x .
\end{aligned}
$$

Since \# $\left(Z_{j}\right) \leq c / \varepsilon_{j}^{n-1}$, we get that

$$
\mathcal{H}^{n-1}\left(\bigcup_{i \in Z_{j}}\left(B_{4 \rho_{j} / 3}^{n-1}\left(x_{i}^{\varepsilon_{j}}\right) \cap \omega\right)\right) \leq c \gamma^{n-1}
$$

and by the equi-integrability of $\left(\left|D u_{j}\right|^{p} / \delta_{j}\right)$ we obtain (4.12). Finally, the weak convergence of $\left(w_{j}\right)$ can be proved as in Lemma 4.2 while the equi-integrability of $\left(\left|D w_{j}\right|^{p} / \delta_{j}\right)$ is just a consequence of the definition of $\left(w_{j}\right)$.

\section{A preliminary analysis of the energy Contribution 'Close' to the CONNECting zones}

For later references, in the following section we study the asymptotic behavior of a sequence of functions which will turn out to represent the energy contribution 'close' to the connecting zones. The results listed in this section will be applied in Section 6 to prove the $\Gamma$-convergence of $\left(\mathcal{F}_{j}\right)$ as well as in Section 7 to compute the explicit formula for $\varphi^{(\ell)}$.

Before starting, let us recall that we consider the domain $\Omega_{j}=\omega^{+\delta_{j}} \cup \omega^{-\delta_{j}} \cup\left(\omega_{r_{j}, \varepsilon_{j}} \times\{0\}\right)$ where $\omega_{r_{j}, \varepsilon_{j}}:=\bigcup_{i \in \mathbb{Z}^{n-1}} B_{r_{j}}^{n-1}\left(x_{i}^{\varepsilon_{j}}\right) \cap \omega$. Our $\Gamma$-convergence analysis deals with the case where the thickness $\delta_{j}$ of $\Omega_{j}$ is much smaller than the period of distribution of the connecting zones $\varepsilon_{j}$; i.e.,

$$
\lim _{j \rightarrow+\infty} \frac{\delta_{j}}{\varepsilon_{j}}=0 .
$$

Moreover, we can exclude that $r_{j} \geq \varepsilon_{j} / 2$ otherwise the zones may overlap. More precisely, we assume that $r_{j} \ll \varepsilon_{j} ;$ i.e.,

$$
\lim _{j \rightarrow+\infty} \frac{r_{j}}{\varepsilon_{j}}=0 .
$$

This choice will be justify a posteriori since (5.1) will be the only admissible assumption to get a non trivial $\Gamma$-convergence result (see Remark 3.4).

Finally, it remains to fix the behavior of $r_{j}$ with respect to $\delta_{j}$. Let us define

$$
\ell:=\lim _{j \rightarrow+\infty} \frac{r_{j}}{\delta_{j}} .
$$

This yields to consider all the possible scenarii, namely to distinguish between the cases: $\ell$ finite, infinite or zero.

For any fixed $\ell \in[0,+\infty]$, we consider the sequence of functions $\left(\varphi_{\gamma, j}^{(\ell)}\right)$ defined in (5.2) and (5.13). Propositions 5.1 and 5.2 establish the existence of the function $\varphi^{(\ell)}$ as the (locally uniform) limit of $\left(\varphi_{\gamma, j}^{(\ell)}\right)$ as $j \rightarrow+\infty$ and $\gamma \rightarrow 0^{+}$while Proposition 5.3 will allow us to prove that $\varphi^{(\ell)}$ is actually the interfacial energy density in $\mathcal{F}^{(\ell)}$ (see e.g. Proposition 6.2). 


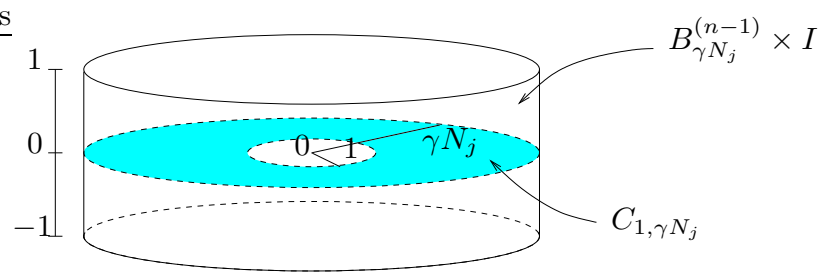

Figure 3. The domain $\left(B_{\gamma N_{j}}^{(n-1)} \times I\right) \backslash C_{1, \gamma N_{j}}$.

5.1. The case $\ell \in(0,+\infty]$. Setting $N_{j}=\varepsilon_{j} / r_{j}$, we define the space

$$
X_{j}^{\gamma}(z):=\left\{\zeta \in W^{1, p}\left(\left(B_{\gamma N_{j}}^{n-1} \times I\right) \backslash C_{1, \gamma N_{j}} ; \mathbb{R}^{m}\right): \zeta=z \text { on }\left(\partial B_{\gamma N_{j}}^{n-1}\right)^{+}, \zeta=0 \text { on }\left(\partial B_{\gamma N_{j}}^{n-1}\right)^{-}\right\},
$$

where $I=(-1,1)$ and we consider the following minimum problem

$$
\varphi_{\gamma, j}^{(\ell)}(z):=\inf \left\{\int_{\left(B_{\gamma N_{j}}^{n-1} \times I\right) \backslash C_{1, \gamma N_{j}}} r_{j}^{p} W\left(r_{j}^{-1} D_{\alpha} \zeta \mid \delta_{j}^{-1} D_{n} \zeta\right) d x: \quad \zeta \in X_{j}^{\gamma}(z)\right\} .
$$

In the next proposition we study the behavior of $\left(\varphi_{\gamma, j}^{(\ell)}\right)$ as $j \rightarrow+\infty$ and $\gamma \rightarrow 0^{+}$.

Proposition 5.1. Let $\ell \in(0,+\infty]$. If

$$
0<R^{(\ell)}:=\lim _{j \rightarrow+\infty} \frac{r_{j}^{n-1-p}}{\varepsilon_{j}^{n-1}}<+\infty
$$

then,

(i) there exists a constant $c>0$ (independent of $j$ and $\gamma$ ) such that

$$
0 \leq \varphi_{\gamma, j}^{(\ell)}(z) \leq c\left(|z|^{p}+\gamma^{n-1}\right)
$$

for all $z \in \mathbb{R}^{m}, j \in \mathbb{N}$ and $\gamma>0$;

(ii) there exists a constant $c>0$ (independent of $j$ and $\gamma$ ) such that

$$
\left|\varphi_{\gamma, j}^{(\ell)}(z)-\varphi_{\gamma, j}^{(\ell)}(w)\right| \leq c|z-w|\left(\gamma^{(n-1)(p-1) / p}+r_{j}^{p-1}+|z|^{p-1}+|w|^{p-1}\right)
$$

for every $z, w \in \mathbb{R}^{m}, j \in \mathbb{N}$ and $\gamma>0$;

(iii) for every fixed $\gamma>0$, up to subsequences, $\varphi_{\gamma, j}^{(\ell)}$ converges locally uniformly on $\mathbb{R}^{m}$ to $\varphi_{\gamma}^{(\ell)}$ as $j \rightarrow+\infty$ and

$$
\left|\varphi_{\gamma}^{(\ell)}(z)-\varphi_{\gamma}^{(\ell)}(w)\right| \leq c|z-w|\left(\gamma^{(n-1)(p-1) / p}+|z|^{p-1}+|w|^{p-1}\right)
$$

for every $z, w \in \mathbb{R}^{m}$;

(iv) up to subsequences, $\varphi_{\gamma}^{(\ell)}$ converges locally uniformly on $\mathbb{R}^{m}$, as $\gamma \rightarrow 0^{+}$, to a continuous function $\varphi^{(\ell)}: \mathbb{R}^{m} \rightarrow[0,+\infty)$ satisfying

$$
0 \leq \varphi^{(\ell)}(z) \leq c|z|^{p}, \quad\left|\varphi^{(\ell)}(z)-\varphi^{(\ell)}(w)\right| \leq c|z-w|\left(|z|^{p-1}+|w|^{p-1}\right)
$$

for every $z, w \in \mathbb{R}^{m}$.

Proof. Fix $\gamma>0$, then $\gamma N_{j}>2$ for $j$ large enough.

(i) According to the $p$-growth condition (3.3),

$$
0 \leq \varphi_{\gamma, j}^{(\ell)}(z) \leq \beta\left(\mathcal{C}_{\gamma, j}(z)+\mathcal{H}^{n-1}\left(B_{1}^{n-1}\right) \gamma^{n-1} \frac{\varepsilon_{j}^{n-1}}{r_{j}^{n-1-p}}\right)
$$


where

$$
\mathcal{C}_{\gamma, j}(z):=\inf \left\{\int_{\left(B_{\gamma N_{j}}^{n-1} \times I\right) \backslash C_{1, \gamma N_{j}}}\left|\left(D_{\alpha} \zeta \mid \frac{r_{j}}{\delta_{j}} D_{n} \zeta\right)\right|^{p} d x: \quad \zeta \in X_{j}^{\gamma}(z)\right\} .
$$

Since $\mathcal{C}_{\gamma, j}(z)$ is invariant by rotations, reasoning as in [2] Section 4.1, we can consider the minimization problem with respect to a particular class of scalar test functions as follows

$$
\begin{array}{r}
\frac{\mathcal{C}_{\gamma, j}(z)}{|z|^{p}}=\inf \left\{\int_{\left(B_{\gamma N_{j}}^{n-1} \times I\right) \backslash C_{1, \gamma N_{j}}}\left|\left(D_{\alpha} \psi \mid \frac{r_{j}}{\delta_{j}} D_{n} \psi\right)\right|^{p} d x: \psi \in W^{1, p}\left(\left(B_{\gamma N_{j}}^{n-1} \times I\right) \backslash C_{1, \gamma N_{j}}\right)\right. \\
\left.\psi=1 \text { on }\left(\partial B_{\gamma N_{j}}^{n-1}\right)^{+} \text {and } \psi=0 \text { on }\left(\partial B_{\gamma N_{j}}^{n-1}\right)^{-}\right\} \\
\leq \inf \left\{\int_{B_{\gamma N_{j}}^{n-1}}\left(\left|D_{\alpha} \psi^{+}\right|^{p}+\left|D_{\alpha} \psi^{-}\right|^{p}\right) d x: \quad\left(\psi^{+}-1\right), \psi^{-} \in W_{0}^{1, p}\left(B_{\gamma N_{j}}^{n-1}\right)\right. \\
\text { and } \left.\psi^{+}=\psi^{-} \text {in } B_{1}^{n-1}\right\} .
\end{array}
$$

Let $\psi_{1}^{ \pm}$be the unique minimizer of the strictly convex minimization problem (5.8). It turns out that $\psi_{2}^{ \pm}:=1-\psi_{1}^{\mp}$ is also a minimizer. Thus by uniqueness, $\psi_{1}^{ \pm}=\psi_{2}^{ \pm}$and in particular, $\psi_{1}^{ \pm}=1 / 2 \mathrm{in} B_{1}^{n-1}$. Hence,

$$
\begin{aligned}
\mathcal{C}_{\gamma, j}(z) \leq|z|^{p} \inf \left\{\int_{B_{\gamma N_{j}}^{n-1}}\left(\left|D_{\alpha} \psi^{+}\right|^{p}+\left|D_{\alpha} \psi^{-}\right|^{p}\right) d x_{\alpha}:\left(\psi^{+}-1\right), \psi^{-} \in W_{0}^{1, p}\left(B_{\gamma N_{j}}^{n-1}\right)\right. \\
=2|z|^{p} \inf \left\{\int_{B_{\gamma N_{j}}^{n-1}}\left|D_{\alpha} \psi\right|^{p} d x_{\alpha}: \quad \psi \in W_{0}^{1, p}\left(B_{\gamma N_{j}}^{n-1}\right) \text { and } \psi=\frac{1}{2} \text { in } B_{1}^{n-1}\right\} \\
=\frac{|z|^{p}}{2^{p-1}} \inf \left\{\int_{B_{\gamma N_{j}}^{n-1}}\left|D_{\alpha} \psi\right|^{p} d x_{\alpha}: \quad \psi \in W_{0}^{1, p}\left(B_{\gamma N_{j}}^{n-1}\right) \text { and } \psi=1 \text { in } B_{1}^{n-1}\right\} \\
=\frac{|z|^{p}}{2^{p-1}} \operatorname{Cap}_{p}\left(B_{1}^{n-1} ; B_{\gamma N_{j}}^{n-1}\right) .
\end{aligned}
$$

Since

$$
\lim _{j \rightarrow+\infty} \operatorname{Cap}_{p}\left(B_{1}^{n-1} ; B_{\gamma N_{j}}^{n-1}\right)=\operatorname{Cap}_{p}\left(B_{1}^{n-1} ; \mathbb{R}^{n-1}\right)<+\infty ;
$$

hence, by (5.3), (5.7) and (5.9) we conclude the proof of (i).

(ii) For every $\eta>0$, there exists $\zeta_{\gamma, j} \in X_{j}^{\gamma}(z)$ such that

$$
\int_{\left(B_{\gamma N_{j}}^{n-1} \times I\right) \backslash C_{1, \gamma N_{j}}} r_{j}^{p} W\left(r_{j}^{-1} D_{\alpha} \zeta_{\gamma, j} \mid \delta_{j}^{-1} D_{n} \zeta_{\gamma, j}\right) d x \leq \varphi_{\gamma, j}^{(\ell)}(z)+\eta .
$$

We want to modify $\zeta_{\gamma, j}$ in order to get an admissible test function for $\varphi_{\gamma, j}^{(\ell)}(w)$. More precisely, we just have to modify $\zeta_{\gamma, j}$ on a neighborhood of $\left(\partial B_{\gamma N_{j}}^{n-1}\right)^{+}$to change the boundary condition $z$ into $w$. To this aim we introduce a cut-off function $\theta \in \mathcal{C}_{c}^{\infty}\left(\mathbb{R}^{n-1} ;[0,1]\right)$, independent of $x_{n}$, such that

$$
\theta\left(x_{\alpha}\right)=\left\{\begin{array}{ll}
1 & \text { if } \quad x_{\alpha} \in B_{1}^{n-1}, \\
0 & \text { if } \quad x_{\alpha} \notin B_{2}^{n-1}
\end{array} \text { and } \quad\left|D_{\alpha} \theta\right| \leq c .\right.
$$


Hence, we define $\tilde{\zeta}_{\gamma, j} \in X_{j}^{\gamma}(w)$ as follows

$$
\tilde{\zeta}_{\gamma, j}=\left\{\begin{array}{lll}
\zeta_{\gamma, j}+\left(1-\theta\left(x_{\alpha}\right)\right)(w-z) & \text { in } & \left(B_{\gamma N_{j}}^{n-1}\right)^{+} \\
\zeta_{\gamma, j} & \text { in } & \left(B_{\gamma N_{j}}^{n-1}\right)^{-} \cup\left(B_{1}^{n-1} \times\{0\}\right) .
\end{array}\right.
$$

By (5.10), since $\zeta_{\gamma, j}=\tilde{\zeta}_{\gamma, j}$ in $\left(B_{\gamma N_{j}}^{n-1}\right)^{-}$, we have that

$$
\begin{aligned}
& \varphi_{\gamma, j}^{(\ell)}(w)-\varphi_{\gamma, j}^{(\ell)}(z) \\
\leq & r_{j}^{p} \int_{\left(B_{\gamma N_{j}}^{n-1} \times I\right) \backslash C_{1, \gamma N_{j}}}\left(W\left(r_{j}^{-1} D_{\alpha} \tilde{\zeta}_{\gamma, j} \mid \delta_{j}^{-1} D_{n} \tilde{\zeta}_{\gamma, j}\right)-W\left(r_{j}^{-1} D_{\alpha} \zeta_{\gamma, j} \mid \delta_{j}^{-1} D_{n} \zeta_{\gamma, j}\right)\right) d x+\eta \\
= & r_{j}^{p} \int_{\left(B_{\gamma N_{j}}^{n-1}\right)^{+}}\left(W\left(r_{j}^{-1} D_{\alpha} \tilde{\zeta}_{\gamma, j} \mid \delta_{j}^{-1} D_{n} \tilde{\zeta}_{\gamma, j}\right)-W\left(r_{j}^{-1} D_{\alpha} \zeta_{\gamma, j} \mid \delta_{j}^{-1} D_{n} \zeta_{\gamma, j}\right)\right) d x+\eta .
\end{aligned}
$$

By (3.4) and Hölder's Inequality, we obtain that

$$
\begin{aligned}
& \varphi_{\gamma, j}^{(\ell)}(w)-\varphi_{\gamma, j}^{(\ell)}(z)-\eta \\
\leq & c \int_{\left(B_{\gamma N_{j}}^{n-1}\right)^{+}}\left(r_{j}^{p-1}+\left|\left(D_{\alpha} \zeta_{\gamma, j} \mid \frac{r_{j}}{\delta_{j}} D_{n} \zeta_{\gamma, j}\right)\right|^{p-1}+\left|\left(D_{\alpha} \tilde{\zeta}_{\gamma, j} \mid \frac{r_{j}}{\delta_{j}} D_{n} \tilde{\zeta}_{\gamma, j}\right)\right|^{p-1}\right) \\
& \quad \times\left|\left(D_{\alpha} \tilde{\zeta}_{\gamma, j}-D_{\alpha} \zeta_{\gamma, j} \mid \frac{r_{j}}{\delta_{j}}\left(D_{n} \tilde{\zeta}_{\gamma, j}-D_{n} \zeta_{\gamma, j}\right)\right)\right| d x \\
\leq & c \int_{\left(B_{\gamma N_{j}}^{n-1}\right)^{+}}\left(r_{j}^{p-1}+2\left|\left(D_{\alpha} \zeta_{\gamma, j} \mid \frac{r_{j}}{\delta_{j}} D_{n} \zeta_{\gamma, j}\right)\right|^{p-1}+\left|D_{\alpha} \theta\right|^{p-1}|w-z|^{p-1}\right)\left|D_{\alpha} \theta\right||w-z| d x \\
\leq \quad & c|z-w|^{p} \int_{B_{\gamma N_{j}}^{n-1}}\left|D_{\alpha} \theta\right|^{p} d x_{\alpha}+c r_{j}^{p-1}|z-w| \int_{B_{\gamma N_{j}}^{n-1}}\left|D_{\alpha} \theta\right| d x_{\alpha} \\
& +2 c|z-w|\left\|D_{\alpha} \theta\right\|_{L^{p}\left(B_{\gamma N_{j}}^{n-1} ; \mathbb{R}^{n-1}\right)}\left\|\left(D_{\alpha} \zeta_{\gamma, j} \mid \frac{r_{j}}{\delta_{j}} D_{n} \zeta_{\gamma, j}\right)\right\|_{L^{p}\left(\left(B_{\gamma N_{j}}^{n-1}\right)^{++; \mathbb{R}^{m \times n}}\right)}^{p-1} .
\end{aligned}
$$

Since $\gamma N_{j}>2$ and $\operatorname{Supp}(\theta) \subset B_{2}^{n-1}$, we obtain that

$$
\begin{aligned}
& \varphi_{\gamma, j}^{(\ell)}(w)-\varphi_{\gamma, j}^{(\ell)}(z) \\
& \quad \leq c|z-w|\left(|z-w|^{p-1}+r_{j}^{p-1}+\left\|\left(D_{\alpha} \zeta_{\gamma, j} \mid \frac{r_{j}}{\delta_{j}} D_{n} \zeta_{\gamma, j}\right)\right\|_{L^{p}\left(\left(B_{\gamma N_{j}}^{n-1}\right)^{+} ; \mathbb{R}^{m \times n}\right)}^{p-1}\right)+\eta .
\end{aligned}
$$

By the $p$-growth condition (3.3), (5.10) and (i), we have that

$$
\begin{aligned}
& \int_{\left(B_{\gamma N_{j}}^{n-1}\right)^{+}}\left|\left(D_{\alpha} \zeta_{\gamma, j} \mid \frac{r_{j}}{\delta_{j}} D_{n} \zeta_{\gamma, j}\right)\right|^{p} d x \\
\leq & \int_{\left(B_{\gamma N_{j}}^{n-1}\right)^{+}} r_{j}^{p} W\left(r_{j}^{-1} D_{\alpha} \zeta_{\gamma, j} \mid \delta_{j}^{-1} D_{n} \zeta_{\gamma, j}\right) d x+r_{j}^{p} \mathcal{H}^{n-1}\left(B_{\gamma N_{j}}^{n-1}\right) \\
\leq & \varphi_{\gamma, j}^{(\ell)}(z)+\eta+c \gamma^{n-1} \frac{\varepsilon_{j}^{n-1}}{r_{j}^{n-1-p}} \\
\leq & c\left(|z|^{p}+\gamma^{n-1}\right)+\eta+c \gamma^{n-1} \frac{\varepsilon_{j}^{n-1}}{r_{j}^{n-1-p}} .
\end{aligned}
$$

Hence, by (5.11), (5.12) and (5.3) we have that

$$
\varphi_{\gamma, j}^{(\ell)}(w)-\varphi_{\gamma, j}^{(\ell)}(z) \leq c|z-w|\left(|z|^{p-1}+|w|^{p-1}+r_{j}^{p-1}+\gamma^{(n-1)(p-1) / p}+\eta^{(p-1) / p}\right)+\eta
$$




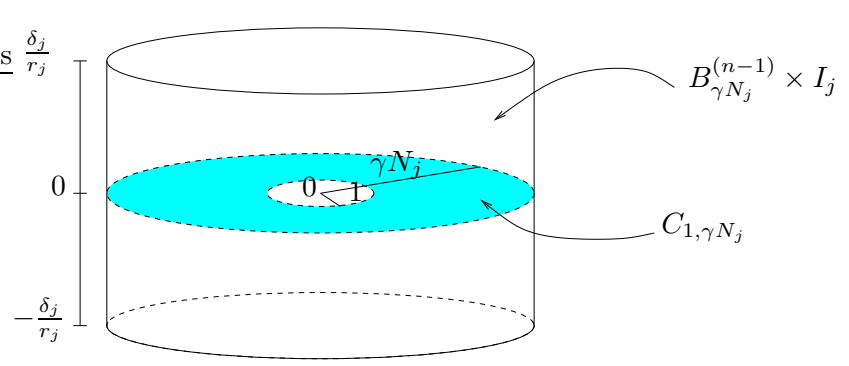

FiguRE 4. The domain $\left(B_{\gamma N_{j}}^{(n-1)} \times I_{j}\right) \backslash C_{1, \gamma N_{j}}$.

and (5.4) follows by the arbitrariness of $\eta$.

By (ii) and Ascoli-Arzela's Theorem we have that, up to subsequences, $\varphi_{\gamma, j}^{(\ell)}$ converges uniformly on compact sets of $\mathbb{R}^{m}$ to $\varphi_{\gamma}^{(\ell)}$ as $j \rightarrow+\infty$. Moreover, passing to the limit in (5.4) as $j \rightarrow+\infty$ we get

$$
\left|\varphi_{\gamma}^{(\ell)}(w)-\varphi_{\gamma}^{(\ell)}(z)\right| \leq c|z-w|\left(|z|^{p-1}+|w|^{p-1}+\gamma^{(n-1)(p-1) / p}\right) .
$$

Hence, we can apply again Ascoli-Arzela's Theorem to conclude that, up to subsequences, $\varphi_{\gamma}^{(\ell)}$ converges uniformly on compact sets of $\mathbb{R}^{m}$ to $\varphi^{(\ell)}$ as $\gamma \rightarrow 0^{+}$. In particular, $\varphi^{(\ell)}: \mathbb{R}^{m} \rightarrow[0,+\infty)$ is a continuous function and

$$
0 \leq \varphi^{(\ell)}(z) \leq c|z|^{p}, \quad\left|\varphi^{(\ell)}(z)-\varphi^{(\ell)}(w)\right| \leq c\left(|z|^{p-1}+|w|^{p-1}\right)|z-w|
$$

for every $z, w \in \mathbb{R}^{m}$.

5.2. The case $\ell=0$. In this case we expect that the energy contribution due to the presence of the sieve is obtained studying the behavior, as $j \rightarrow+\infty$ and $\gamma \rightarrow 0^{+}$, of the sequence $\left(\varphi_{\gamma, j}^{(0)}\right)$ defined as follows

$$
\begin{aligned}
\varphi_{\gamma, j}^{(0)}(z) & :=\frac{\delta_{j}}{r_{j}} \inf \left\{\int_{\left(B_{\gamma N_{j}}^{n-1} \times I\right) \backslash C_{1, \gamma N_{j}}} r_{j}^{p} W\left(r_{j}^{-1} D_{\alpha} \zeta \mid \delta_{j}^{-1} D_{n} \zeta\right) d x: \quad \zeta \in X_{j}^{\gamma}(z)\right\} \\
& =\inf \left\{\int_{\left(B_{\gamma N_{j}}^{n-1} \times I_{j}\right) \backslash C_{1, \gamma N_{j}}} r_{j}^{p} W\left(r_{j}^{-1} D \zeta\right) d x: \quad \zeta \in Y_{j}^{\gamma}(z)\right\}
\end{aligned}
$$

where $I_{j}:=\left(-\delta_{j} / r_{j}, \delta_{j} / r_{j}\right)$ and

$$
\begin{aligned}
Y_{j}^{\gamma}(z)=\left\{\zeta \in W^{1, p}\left(\left(B_{\gamma N_{j}}^{n-1} \times I_{j}\right) \backslash C_{1, \gamma N_{j}} ; \mathbb{R}^{m}\right):\right. & \zeta=z \text { on }\left(\partial B_{\gamma N_{j}}^{n-1}\right)^{+\left(\delta_{j} / r_{j}\right)}, \\
& \left.\zeta=0 \text { on }\left(\partial B_{\gamma N_{j}}^{n-1}\right)^{-\left(\delta_{j} / r_{j}\right)}\right\} .
\end{aligned}
$$

Note that in this case we are interested in the limit behavior of a sequence that is obtained from the one corresponding to $\ell \in(0,+\infty]$ multiplying it by $\delta_{j} / r_{j}$ (see (5.13) and recall (5.2)). Let us try to motivate this choice.

Let $\ell \in(0,+\infty)$, then starting from (5.2) by a change of variable it is immediate to check that

$$
\varphi_{\gamma, j}^{(\ell)}(z)=\frac{r_{j}}{\delta_{j}} \inf \left\{\int_{\left(B_{\gamma N_{j}}^{n-1} \times I_{j}\right) \backslash C_{1, \gamma N_{j}}} r_{j}^{p} W\left(r_{j}^{-1} D \zeta\right) d x: \quad \zeta \in Y_{j}^{\gamma}(z)\right\} .
$$

Now assuming that $\lim _{j \rightarrow+\infty} r_{j}^{n-p} /\left(\delta_{j} \varepsilon_{j}^{n-1}\right)<+\infty$ (or equivalently that $\lim _{j \rightarrow+\infty} r_{j}^{n-1-p} / \varepsilon_{j}^{n-1}<+\infty$; see Remark 3.5) we know that the sequence $\left(\varphi_{\gamma, j}^{(\ell)}\right)$ converges to $\ell \tilde{\varphi}^{(\ell)}$, for some $\tilde{\varphi}^{(\ell)}$, locally uniformly 
in $\mathbb{R}^{m}$, as $j \rightarrow+\infty$ and $\gamma \rightarrow 0^{+}$(Proposition 5.1). Then if $\ell \in(0,+\infty)$, studying the limit behavior of (5.13) is perfectly equivalent to study the limit behavior of (5.2). While if $\ell=\lim _{j \rightarrow+\infty} r_{j} / \delta_{j}=0,(5.14)$ suggests that, to recover nontrivial information in the limit, we have to study the asymptotic behavior of the sequence obtained from (5.14) dividing it by $r_{j} / \delta_{j}$, that is to study the asymptotic behavior of the sequence given by (5.13).

Following the line of the proof of Proposition 5.1, we want to establish an analogous result for the sequence $\left(\varphi_{\gamma, j}^{(0)}\right)$.

Proposition 5.2. Let $\ell=0$. If

$$
0<R^{(0)}=\lim _{j \rightarrow+\infty} \frac{r_{j}^{n-p}}{\varepsilon_{j}^{n-1} \delta_{j}}<+\infty
$$

then,

(i) there exists a constant $c>0$ (independent of $j$ and $\gamma$ ) such that

$$
0 \leq \varphi_{\gamma, j}^{(0)}(z) \leq c\left(|z|^{p}+\gamma^{n-1}\right)
$$

for all $z \in \mathbb{R}^{m}, j \in \mathbb{N}$ and $\gamma>0$;

(ii) there exists a constant $c>0$ (independent of $j$ and $\gamma$ ) such that

$$
\left|\varphi_{\gamma, j}^{(0)}(z)-\varphi_{\gamma, j}^{(0)}(w)\right| \leq c|z-w|\left(\gamma^{(n-1)(p-1) / p}+r_{j}^{n-1}+|z|^{p-1}+|w|^{p-1}\right)
$$

for every $z, w \in \mathbb{R}^{m}, j \in \mathbb{N}$ and $\gamma>0$;

(iii) for every fixed $\gamma>0$, up to subsequences, $\varphi_{\gamma, j}^{(0)}$ converges locally uniformly in $\mathbb{R}^{m}$ to $\varphi_{\gamma}^{(0)}$ as $j \rightarrow+\infty$, and

$$
\left|\varphi_{\gamma}^{(0)}(z)-\varphi_{\gamma}^{(0)}(w)\right| \leq c|z-w|\left(\gamma^{(n-1)(p-1) / p}+|z|^{p-1}+|w|^{p-1}\right)
$$

for every $z, w \in \mathbb{R}^{m}$;

(iv) up to subsequences, $\varphi_{\gamma}^{(0)}$ converges locally uniformly in $\mathbb{R}^{m}$, as $\gamma \rightarrow 0^{+}$, to a continuous function $\varphi^{(0)}: \mathbb{R}^{m} \rightarrow[0,+\infty)$ satisfying

$$
0 \leq \varphi^{(0)}(z) \leq c|z|^{p}, \quad\left|\varphi^{(0)}(z)-\varphi^{(0)}(w)\right| \leq c|z-w|\left(|z|^{p-1}+|w|^{p-1}\right)
$$

for every $z, w \in \mathbb{R}^{m}$.

Proof. Fix $\gamma>0$, then $\gamma N_{j}>2$ and $\delta_{j} / r_{j}>2$ for $j$ large enough.

(i) According to the $p$-growth condition (3.3),

$$
0 \leq \varphi_{\gamma, j}^{(0)}(z) \leq \beta\left(\mathcal{C}_{\gamma, j}(z)+2 \mathcal{H}^{n-1}\left(B_{1}^{n-1}\right) \gamma^{n-1} \frac{\delta_{j} \varepsilon_{j}^{n-1}}{r_{j}^{n-p}}\right)
$$

where

$$
\mathcal{C}_{\gamma, j}(z)=\inf \left\{\int_{\left(B_{\gamma N_{j}}^{n-1} \times I_{j}\right) \backslash C_{1, \gamma N_{j}}}|D \zeta|^{p} d x: \quad \zeta \in Y_{j}^{\gamma}(z)\right\} .
$$

Arguing similarly than in the proof of Proposition 5.1, we can rewrite

$$
\begin{array}{r}
\frac{\mathcal{C}_{\gamma, j}(z)}{|z|^{p}}=\inf \left\{\int_{\left(B_{\gamma N_{j}}^{n-1} \times I_{j}\right) \backslash C_{1, \gamma N_{j}}}|D \psi|^{p} d x: \psi \in W^{1, p}\left(\left(B_{\gamma N_{j}}^{n-1} \times I_{j}\right) \backslash C_{1, \gamma N_{j}}\right),\right. \\
\left.\psi=1 \text { on }\left(\partial B_{\gamma N_{j}}^{n-1}\right)^{+\left(\delta_{j} / r_{j}\right)}, \quad \psi=0 \text { on }\left(\partial B_{\gamma N_{j}}^{n-1}\right)^{-\left(\delta_{j} / r_{j}\right)}\right\} .
\end{array}
$$


Let $\psi_{1}$ be the unique minimizer of the strictly convex minimization problem (5.20). It turns out that $\psi_{2}\left(x_{\alpha}, x_{n}\right):=1-\psi_{1}\left(x_{\alpha},-x_{n}\right)$ is also a minimizer. Thus by uniqueness, $\psi_{1}=\psi_{2}$ and in particular, $\psi_{1}=\psi_{2}=1 / 2$ on $B_{1}^{n-1} \times\{0\}$. Thus

$$
\begin{gathered}
\mathcal{C}_{\gamma, j}(z)=2|z|^{p} \inf \left\{\int_{\left(B_{\gamma N_{j}}^{n-1}\right)^{+\left(\delta_{j} / r_{j}\right)}}|D \psi|^{p} d x: \quad \psi \in W^{1, p}\left(\left(B_{\gamma N_{j}}^{n-1}\right)^{+\left(\delta_{j} / r_{j}\right)}\right),\right. \\
\left.\psi=0 \text { on }\left(\partial B_{\gamma N_{j}}^{n-1}\right)^{+\left(\delta_{j} / r_{j}\right)} \text { and } \psi=\frac{1}{2} \text { on } B_{1}^{n-1} \times\{0\}\right\} \\
=\frac{|z|^{p}}{2^{p-1}} \inf \left\{\int_{\left(B_{\gamma N_{j}}^{n-1}\right)^{+\left(\delta_{j} / r_{j}\right)}}|D \psi|^{p} d x: \quad \psi \in W^{1, p}\left(\left(B_{\gamma N_{j}}^{n-1}\right)^{+\left(\delta_{j} / r_{j}\right)}\right),\right. \\
\left.\psi=0 \text { on }\left(\partial B_{\gamma N_{j}}^{n-1}\right)^{+\left(\delta_{j} / r_{j}\right)} \text { and } \psi=1 \text { on } B_{1}^{n-1} \times\{0\}\right\} \\
\leq \frac{|z|^{p}}{2^{p}} \operatorname{Cap}_{p}\left(B_{1}^{n-1} ; B_{\gamma N_{j}}^{n-1} \times I_{j}\right) .
\end{gathered}
$$

Since

$$
\lim _{j \rightarrow+\infty} \operatorname{Cap}_{p}\left(B_{1}^{n-1} ; B_{\gamma N_{j}}^{n-1} \times I_{j}\right)=\operatorname{Cap}_{p}\left(B_{1}^{n-1} ; \mathbb{R}^{n}\right)<+\infty ;
$$

hence, by (5.15), (5.19) and (5.21) we conclude the proof of (i).

(ii) We can proceed as in the proof of Proposition 5.1 (ii) using a different cut-off function also depending on $x_{n}$. Namely, let $\theta \in \mathcal{C}_{c}^{\infty}\left(\mathbb{R}^{n} ;[0,1]\right)$ be such that

$$
\theta\left(x_{\alpha}, x_{n}\right)=\left\{\begin{array}{lll}
1 & \text { if } & \left(x_{\alpha}, x_{n}\right) \in B_{1}^{n-1} \times(-1,1), \\
0 & \text { if } & \left(x_{\alpha}, x_{n}\right) \notin B_{2}^{n-1} \times(-2,2)
\end{array} \quad \text { and } \quad|D \theta| \leq c .\right.
$$

Hence, if $\zeta_{\gamma, j} \in Y_{j}^{\gamma}(z)$ is a sequence which 'almost attains' the infimum value $\varphi_{\gamma, j}^{(0)}$, we define $\tilde{\zeta}_{\gamma, j} \in Y_{j}^{\gamma}(w)$ as follows

$$
\tilde{\zeta}_{\gamma, j}= \begin{cases}\zeta_{\gamma, j}+(1-\theta(x))(w-z) & \text { in }\left(B_{\gamma N_{j}}^{n-1}\right)^{+\left(\delta_{j} / r_{j}\right)}, \\ \zeta_{\gamma, j} & \text { in }\left(\left(B_{\gamma N_{j}}^{n-1}\right)^{-\left(\delta_{j} / r_{j}\right)}\right) \cup\left(B_{1}^{n-1} \times\{0\}\right) .\end{cases}
$$

By (5.15) we conclude the proof of (ii) reasoning as in the proof of Proposition 5.1 (ii).

The proof of (iii) and (iv) follows the line of the proof of (iii) and (iv) in Proposition 5.1.

Now we are able to describe the energy contribution close to the connecting zones as $j \rightarrow+\infty$ and $\gamma \rightarrow 0^{+}$.

Proposition 5.3 (Discrete approximation of the interfacial energy). Let $\left(u_{j}\right) \subset W^{1, p}\left(\Omega_{j} ; \mathbb{R}^{m}\right) \cap L^{\infty}\left(\Omega_{j} ; \mathbb{R}^{m}\right)$ be a sequence converging to $\left(u^{+}, u^{-}\right) \in W^{1, p}\left(\omega ; \mathbb{R}^{m}\right) \times W^{1, p}\left(\omega ; \mathbb{R}^{m}\right)$ such that $\sup _{j} \mathcal{F}_{j}\left(u_{j}\right)<+\infty$ and satisfying $\sup _{j \in \mathbb{N}}\left\|u_{j}\right\|_{L^{\infty}\left(\Omega_{j} ; \mathbb{R}^{m}\right)}<+\infty$. Let $\left(u_{j}^{i \pm}\right)$ be as in (4.1). If

$$
\ell \in(0,+\infty] \quad \text { and } \quad 0<R^{(\ell)}=\lim _{j \rightarrow+\infty} \frac{r_{j}^{n-1-p}}{\varepsilon_{j}^{n-1}}<+\infty
$$

or

$$
\ell=0 \quad \text { and } \quad 0<R^{(0)}=\lim _{j \rightarrow+\infty} \frac{r_{j}^{n-p}}{\delta_{j} \varepsilon_{j}^{n-1}}<+\infty
$$

then

$$
\lim _{\gamma \rightarrow 0^{+}} \limsup _{j \rightarrow+\infty} \int_{\omega}\left|\sum_{i \in Z_{j}} \varphi_{\gamma, j}^{(\ell)}\left(u_{j}^{i+}-u_{j}^{i-}\right) \chi_{Q_{i, \varepsilon_{j}}^{n-1}}-\varphi^{(\ell)}\left(u^{+}-u^{-}\right)\right| d x_{\alpha}=0,
$$

for every $\ell \in[0,+\infty]$. 
THE NEUMANN SIEVE PROBLEM AND DIMENSIONAL REDUCTION

Proof. Since $\sup _{j \in \mathbb{N}}\left\|u_{j}\right\|_{L^{\infty}\left(\Omega_{j} ; \mathbb{R}^{m}\right)}<+\infty$ by Propositions 5.1 or 5.2 we have that

$$
\begin{aligned}
& \limsup _{j \rightarrow+\infty} \int_{\omega}\left|\sum_{i \in Z_{j}} \varphi_{\gamma, j}^{(\ell)}\left(u_{j}^{i+}-u_{j}^{i-}\right) \chi_{Q_{i, \varepsilon_{j}}^{n-1}}-\varphi^{(\ell)}\left(u^{+}-u^{-}\right)\right| d x_{\alpha} \\
\leq & \limsup _{j \rightarrow+\infty} \int_{\omega} \sum_{i \in Z_{j}}\left|\varphi_{\gamma, j}^{(\ell)}\left(u_{j}^{i+}-u_{j}^{i-}\right)-\varphi^{(\ell)}\left(u_{j}^{i+}-u_{j}^{i-}\right)\right| \chi_{Q_{i, \varepsilon_{j}}^{n-1}} d x_{\alpha} \\
& +\limsup _{j \rightarrow+\infty} \int_{\omega}\left|\sum_{i \in Z_{j}} \varphi^{(\ell)}\left(u_{j}^{i+}-u_{j}^{i-}\right) \chi_{Q_{i, \varepsilon_{j}}^{n-1}}-\varphi^{(\ell)}\left(u^{+}-u^{-}\right)\right| d x_{\alpha} \\
\leq \quad & o(1)+\limsup _{j \rightarrow+\infty} \int_{\omega}\left|\sum_{i \in Z_{j}} \varphi^{(\ell)}\left(u_{j}^{i+}-u_{j}^{i-}\right) \chi_{Q_{i, \varepsilon_{j}}^{n-1}}-\varphi^{(\ell)}\left(u^{+}-u^{-}\right)\right| d x_{\alpha},
\end{aligned}
$$

as $\gamma \rightarrow 0^{+}$. By (5.6) or (5.18) and Hölder's Inequality we have that

$$
\begin{aligned}
& \limsup _{j \rightarrow+\infty} \int_{\omega}\left|\sum_{i \in Z_{j}} \varphi^{(\ell)}\left(u_{j}^{i+}-u_{j}^{i-}\right) \chi_{Q_{i, \varepsilon_{j}}^{n-1}}-\varphi^{(\ell)}\left(u^{+}-u^{-}\right)\right| d x_{\alpha} \\
= & \limsup _{j \rightarrow+\infty} \sum_{i \in Z_{j}} \int_{Q_{i, \varepsilon_{j}}^{n-1}}\left|\varphi^{(\ell)}\left(u_{j}^{i+}-u_{j}^{i-}\right)-\varphi^{(\ell)}\left(u^{+}-u^{-}\right)\right| d x_{\alpha} \\
\leq & c \limsup _{j \rightarrow+\infty}\left(\sum_{i \in Z_{j}} \int_{Q_{i, \varepsilon_{j}}^{n-1}}\left|u_{j}^{i+}-u^{+}\right|^{p}+\left|u_{j}^{i-}-u^{-}\right|^{p} d x_{\alpha}\right)^{1 / p} .
\end{aligned}
$$

Hence, it remains to prove that

$$
\limsup _{j \rightarrow+\infty} \sum_{i \in Z_{j}} \int_{Q_{i, \varepsilon_{j}}^{n-1}}\left|u^{ \pm}-u_{j}^{i \pm}\right|^{p} d x_{\alpha}=0
$$

By Lemma 4.1 (ii) applied with $\rho=\varepsilon_{j}, B_{\rho}=C_{j}^{i}$ and $A_{\rho}=Q_{i, \varepsilon_{j}}^{n-1}$ and since $\delta_{j} \ll \varepsilon_{j}$, we have

$$
\begin{aligned}
\int_{Q_{i, \varepsilon_{j}}^{n-1}}\left|u^{ \pm}-u_{j}^{i \pm}\right|^{p} d x_{\alpha} & \leq \frac{c}{\delta_{j}}\left(\int_{\left(Q_{i, \varepsilon_{j}}^{n-1}\right)^{ \pm \delta_{j}}}\left|u_{j}-u^{ \pm}\right|^{p} d x+\int_{\left(Q_{i, \varepsilon_{j}}^{n-1}\right)^{ \pm \delta_{j}}}\left|u_{j}-u_{j}^{i \pm}\right|^{p} d x\right) \\
& \leq \frac{c}{\delta_{j}} \int_{\left(Q_{i, \varepsilon_{j}}^{n-1}\right)^{ \pm \delta_{j}}}\left|u_{j}-u^{ \pm}\right|^{p} d x+\frac{c \varepsilon_{j}^{p}}{\delta_{j}} \int_{\left(Q_{i, \varepsilon_{j}}^{n-1}\right)^{ \pm \delta_{j}}}\left|D u_{j}\right|^{p} d x,
\end{aligned}
$$

for all $i \in Z_{j}$; hence, summing up on $i \in Z_{j}$, we find

$$
\sum_{i \in Z_{j}} \int_{Q_{i, \varepsilon_{j}}^{n-1}}\left|u_{j}-u_{j}^{i \pm}\right|^{p} d x_{\alpha} \leq \frac{c}{\delta_{j}} \int_{\omega^{ \pm \delta_{j}}}\left|u_{j}-u^{ \pm}\right|^{p} d x+\frac{c \varepsilon_{j}^{p}}{\delta_{j}} \int_{\omega^{ \pm \delta_{j}}}\left|D u_{j}\right|^{p} d x
$$

then passing to the limit as $j \rightarrow+\infty$ by the convergence of $\left(u_{j}\right)$ towards $\left(u^{+}, u^{-}\right)$and $\sup _{j} \mathcal{F}_{j}\left(u_{j}\right)<+\infty$ we get (5.23) and then (5.22).

\section{6. $\Gamma$-CONVERGENCE RESUlT}

6.1. The liminf inequality. Let $\left(u_{j}\right) \subset W^{1, p}\left(\Omega_{j} ; \mathbb{R}^{m}\right) \cap L^{\infty}\left(\Omega_{j} ; \mathbb{R}^{m}\right)$ be a sequence converging to $\left(u^{+}, u^{-}\right) \in W^{1, p}\left(\omega, \mathbb{R}^{m}\right) \times W^{1, p}\left(\omega, \mathbb{R}^{m}\right)$ such that $\sup _{j \in \mathbb{N}}\left\|u_{j}\right\|_{L^{\infty}\left(\Omega_{j} ; \mathbb{R}^{m}\right)}<+\infty$ and

$$
\liminf _{j \rightarrow+\infty} \mathcal{F}_{j}\left(u_{j}\right)<+\infty .
$$


By Lemma 4.2 , for every fixed $k \in \mathbb{N}$, there exists a sequence $\left(w_{j}\right) \subset W^{1, p}\left(\Omega_{j} ; \mathbb{R}^{m}\right) \cap L^{\infty}\left(\Omega_{j} ; \mathbb{R}^{m}\right)$ weakly converging to $\left(u^{+}, u^{-}\right)$satisfying $(4.2),(4.3)$ and such that

$$
\begin{aligned}
& \liminf _{j \rightarrow+\infty} \frac{1}{\delta_{j}}\left(\int_{\omega^{+\delta_{j}}} W\left(D u_{j}\right) d x+\int_{\omega^{-\delta_{j}}} W\left(D u_{j}\right) d x\right) \\
\geq & \liminf _{j \rightarrow+\infty} \frac{1}{\delta_{j}}\left(\int_{\omega^{+\delta_{j}}} W\left(D w_{j}\right) d x+\int_{\omega^{-\delta_{j}}} W\left(D w_{j}\right) d x\right)-\frac{c}{k} \\
\geq & \liminf _{j \rightarrow+\infty} \frac{1}{\delta_{j}}\left(\int_{\left(\omega \backslash E_{j}\right)^{+\delta_{j}}} W\left(D w_{j}\right) d x+\int_{\left(\omega \backslash E_{j}\right)^{-\delta_{j}}} W\left(D w_{j}\right) d x\right) \\
& +\liminf _{j \rightarrow+\infty} \frac{1}{\delta_{j}}\left(\int_{E_{j}^{+\delta_{j}}} W\left(D w_{j}\right) d x+\int_{E_{j}^{-\delta_{j}}} W\left(D w_{j}\right) d x\right)-\frac{c}{k},
\end{aligned}
$$

where $E_{j}:=\bigcup_{i \in Z_{j}} B_{\rho_{j}^{i}}^{n-1}\left(x_{i}^{\varepsilon_{j}}\right)$.

We first consider the energy contribution 'far' from the connecting zones. In this case, we suitably modify the sequence $\left(w_{j}\right)$ in order to get a constant inside each half cylinder $B_{\rho_{j}^{i}}^{(n-1)}\left(x_{i}^{\varepsilon_{j}}\right)^{ \pm \delta_{j}}$. Then, we apply the classical result of dimensional reduction proved in [23] to $\omega^{+\delta_{j}}$ and $\omega^{-\delta_{j}}$, separately.

Proposition 6.1. We have

$$
\begin{aligned}
& \liminf _{j \rightarrow+\infty} \frac{1}{\delta_{j}}\left(\int_{\left(\omega \backslash E_{j}\right)^{+\delta_{j}}} W\left(D w_{j}\right) d x+\int_{\left(\omega \backslash E_{j}\right)^{-\delta_{j}}} W\left(D w_{j}\right) d x\right) \\
& \geq \int_{\omega}\left(\mathcal{Q}_{n-1} \bar{W}\left(D_{\alpha} u^{+}\right)+\mathcal{Q}_{n-1} \bar{W}\left(D_{\alpha} u^{-}\right)\right) d x_{\alpha} .
\end{aligned}
$$

Proof. We define

$$
v_{j}:=\left\{\begin{array}{lll}
w_{j} & \text { in } & \left(\omega \backslash E_{j}\right)^{ \pm \delta_{j}} \\
u_{j}^{i \pm} & \text { in } & B_{\rho_{j}^{i}}^{n-1}\left(x_{i}^{\varepsilon_{j}}\right)^{ \pm \delta_{j}} \text { if } i \in Z_{j} .
\end{array}\right.
$$

Then $\left(v_{j}\right) \subset W^{1, p}\left(\Omega_{j} ; \mathbb{R}^{m}\right)$ converges weakly to $\left(u^{+}, u^{-}\right)$. In fact,

$$
\sup _{j \in \mathbb{N}} \frac{1}{\delta_{j}} \int_{\omega^{ \pm \delta_{j}}}\left|D v_{j}\right|^{p} d x \leq \sup _{j \in \mathbb{N}} \frac{1}{\delta_{j}} \int_{\omega^{ \pm \delta_{j}}}\left|D u_{j}\right|^{p} d x<+\infty
$$

Moreover, since $\rho_{j}^{i}<\rho_{j}<\varepsilon_{j} / 2$, then $B_{\rho_{j}^{i}}^{n-1}\left(x_{i}^{\varepsilon_{j}}\right) \subset Q_{i, \varepsilon_{j}}^{n-1}$; hence,

$$
\int_{\omega^{ \pm \delta_{j}}}\left|v_{j}-u^{ \pm}\right|^{p} d x \leq \int_{\left(\omega \backslash E_{j}\right)^{ \pm \delta_{j}}}\left|w_{j}-u^{ \pm}\right|^{p} d x+\sum_{i \in Z_{j}} \int_{\left(Q_{i, \varepsilon_{j}}^{n-1}\right)^{ \pm \delta_{j}}}\left|u^{ \pm}-u_{j}^{i \pm}\right|^{p} d x
$$

and, by (5.24), we obtain that

$$
\begin{aligned}
\frac{1}{\delta_{j}} \int_{\omega^{ \pm \delta_{j}}}\left|v_{j}-u^{ \pm}\right|^{p} d x \leq & \frac{1}{\delta_{j}} \int_{\omega^{ \pm \delta_{j}}}\left|w_{j}-u^{ \pm}\right|^{p} d x+\frac{c}{\delta_{j}} \int_{\omega^{ \pm \delta_{j}}}\left|u_{j}-u^{ \pm}\right|^{p} d x \\
& +c \varepsilon_{j}^{p} \sup _{j \in \mathbb{N}} \frac{1}{\delta_{j}} \int_{\omega^{ \pm \delta_{j}}}\left|D u_{j}\right|^{p} d x .
\end{aligned}
$$

Passing to the limit as $j \rightarrow+\infty$ in (6.4), by (6.3) and Remark 3.2 we get that $\left(v_{j}\right)$ converges weakly to $\left(u^{+}, u^{-}\right)$. 
Since $W(0)=0$, by $(6.2)$ and [23] Theorem 2, we have

$$
\begin{aligned}
& \liminf _{j \rightarrow+\infty} \frac{1}{\delta_{j}}\left(\int_{\left(\omega \backslash E_{j}\right)^{+\delta_{j}}} W\left(D w_{j}\right) d x+\int_{\left(\omega \backslash E_{j}\right)^{-\delta_{j}}} W\left(D w_{j}\right) d x\right) \\
= & \liminf _{j \rightarrow+\infty} \frac{1}{\delta_{j}}\left(\int_{\left(\omega \backslash E_{j}\right)^{+\delta_{j}}} W\left(D v_{j}\right) d x+\int_{\left(\omega \backslash E_{j}\right)^{-\delta_{j}}} W\left(D v_{j}\right) d x\right) \\
= & \liminf _{j \rightarrow+\infty} \frac{1}{\delta_{j}}\left(\int_{\omega^{+\delta_{j}}} W\left(D v_{j}\right) d x+\int_{\omega^{-\delta_{j}}} W\left(D v_{j}\right) d x\right) \\
\geq & \int_{\omega} \mathcal{Q}_{n-1} \bar{W}\left(D_{\alpha} u^{+}\right) d x_{\alpha}+\int_{\omega} \mathcal{Q}_{n-1} \bar{W}\left(D_{\alpha} u^{-}\right) d x_{\alpha} .
\end{aligned}
$$

Now let us deal with the contribution 'near' the connecting zones. We always work under the assumption

or

$$
\ell \in(0,+\infty] \quad \text { and } \quad 0<R^{(\ell)}=\lim _{j \rightarrow+\infty} \frac{r_{j}^{(n-1-p)}}{\varepsilon_{j}^{n-1}}<+\infty
$$

$$
\ell=0 \quad \text { and } \quad 0<R^{(0)}=\lim _{j \rightarrow+\infty} \frac{r_{j}^{(n-p)}}{\delta_{j} \varepsilon_{j}^{n-1}}<+\infty .
$$

In the following proposition we suitably modify $\left(w_{j}\right)$ in each surrounding cylinder in order to get an admissible test function for the minimum problem (5.2) or (5.13).

Proposition 6.2. Let $\ell \in[0,+\infty]$. Then

$$
\liminf _{j \rightarrow+\infty} \frac{1}{\delta_{j}}\left(\int_{E_{j}^{+\delta_{j}}} W\left(D w_{j}\right) d x+\int_{E_{j}^{-\delta_{j}}} W\left(D w_{j}\right) d x\right) \geq R^{(\ell)} \int_{\omega} \varphi^{(\ell)}\left(u^{+}-u^{-}\right) d x_{\alpha}+o(1),
$$

as $\gamma \rightarrow 0^{+}$.

Proof. Let $\ell \in(0,+\infty]$, the case $\ell=0$ can be treated similarly. Let $i \in Z_{j}$ and $N_{j}=\frac{\varepsilon_{j}}{r_{j}}$. Since $\rho_{j}^{i}<\gamma \varepsilon_{j}$, we can define

$$
\zeta_{j}^{i}:= \begin{cases}w_{j}\left(x_{i}^{\varepsilon_{j}}+r_{j} y_{\alpha}, \delta_{j} y_{n}\right)-u_{j}^{i-} & \text { in }\left(B_{\rho_{j}^{i} / r_{j}}^{n-1} \times I\right) \backslash C_{1, \rho_{j}^{i} / r_{j}} \\ \left(u_{j}^{i+}-u_{j}^{i-}\right) & \text { in }\left(B_{\gamma N_{j}}^{n-1} \backslash B_{\rho_{j}^{i} / r_{j}}^{n-1}\right)^{+} \\ 0 & \text { in }\left(B_{\gamma N_{j}}^{n-1} \backslash B_{\rho_{j}^{i} / r_{j}}^{n-1}\right)^{-},\end{cases}
$$

where $N_{j}=\varepsilon_{j} / r_{j}$. Then $\zeta_{j}^{i} \in W^{1, p}\left(\left(B_{\gamma N_{j}}^{n-1} \times I\right) \backslash C_{1, \gamma N_{j}} ; \mathbb{R}^{m}\right), \zeta_{j}^{i}=\left(u_{j}^{i+}-u_{j}^{i-}\right)$ on $\left(\partial B_{\gamma N_{j}}^{n-1}\right)^{+}$and $\zeta_{j}^{i}=0$ on $\left(\partial B_{\gamma N_{j}}^{n-1}\right)^{-}$. Since $W(0)=0$, changing variable, by (5.2) we get

$$
\begin{aligned}
& \frac{1}{\delta_{j}}\left(\int_{B_{\rho_{j}^{i}}^{n-1}\left(x_{i}^{\varepsilon_{j}}\right)^{+\delta_{j}}} W\left(D w_{j}\right) d x+\int_{B_{\rho_{j}^{i}}^{n-1}\left(x_{i}^{\varepsilon_{j}}\right)^{-\delta_{j}}} W\left(D w_{j}\right) d x\right) \\
= & r_{j}^{n-1}\left(\int_{\left(B_{\rho_{j}^{i} / r_{j}}^{n-1}\right)^{+}} W\left(r_{j}^{-1} D_{\alpha} \zeta_{j}^{i} \mid \delta_{j}^{-1} D_{n} \zeta_{j}^{i}\right) d y+\int_{\left(B_{\rho_{j}^{i} / r_{j}}^{n-1}\right)^{-}} W\left(r_{j}^{-1} D_{\alpha} \zeta_{j}^{i} \mid \delta_{j}^{-1} D_{n} \zeta_{j}^{i}\right) d y\right) \\
= & r_{j}^{n-1} \int_{\left(B_{\gamma N_{j}}^{n-1} \times I\right) \backslash C_{1, \gamma N_{j}}} W\left(r_{j}^{-1} D_{\alpha} \zeta_{j}^{i} \mid \delta_{j}^{-1} D_{n} \zeta_{j}^{i}\right) d y \\
\geq & r_{j}^{n-1-p} \varphi_{\gamma, j}^{(\ell)}\left(u_{j}^{i+}-u_{j}^{i-}\right) .
\end{aligned}
$$


Summing up in (6.5), for $i \in Z_{j}$, we get that

$$
\begin{aligned}
& \frac{1}{\delta_{j}}\left(\int_{E_{j}^{+\delta_{j}}} W\left(D w_{j}\right) d x+\int_{E_{j}^{-\delta_{j}}} W\left(D w_{j}\right) d x\right) \\
= & \sum_{i \in Z_{j}} \frac{1}{\delta_{j}}\left(\int_{B_{\rho_{j}^{i}}^{n-1}\left(x_{i}^{\varepsilon_{j}}\right)^{+\delta_{j}}} W\left(D w_{j}\right) d x+\int_{B_{\rho_{j}^{i}}^{n-1}\left(x_{i}^{\varepsilon_{j}}\right)^{-\delta_{j}}} W\left(D w_{j}\right) d x\right) \\
\geq & r_{j}^{n-1-p} \sum_{i \in Z_{j}} \varphi_{\gamma, j}^{(\ell)}\left(u_{j}^{i+}-u_{j}^{i-}\right)=\frac{r_{j}^{n-1-p}}{\varepsilon_{j}^{n-1}} \sum_{i \in Z_{j}} \varepsilon_{j}^{n-1} \varphi_{\gamma, j}^{(\ell)}\left(u_{j}^{i+}-u_{j}^{i-}\right) .
\end{aligned}
$$

Passing to the limit as $j \rightarrow+\infty$ we get, by (5.3) and Proposition 5.3, that

$$
\begin{aligned}
& \liminf _{j \rightarrow+\infty} \frac{1}{\delta_{j}}\left(\int_{E_{j}^{+\delta_{j}}} W\left(D w_{j}\right) d x+\int_{E_{j}^{-\delta_{j}}} W\left(D w_{j}\right) d x\right) \\
\geq & R^{(\ell)} \int_{\omega} \varphi^{(\ell)}\left(u^{+}-u^{-}\right) d x_{\alpha} \\
& +R^{(\ell)} \liminf _{j \rightarrow+\infty} \int_{\omega}\left(\sum_{i \in Z_{j}} \varphi_{\gamma, j}^{(\ell)}\left(u_{j}^{i+}-u_{j}^{i-}\right) \chi_{Q_{i, \varepsilon_{j}}^{n-1}}-\varphi^{(\ell)}\left(u^{+}-u^{-}\right)\right) d x_{\alpha} \\
= & R^{(\ell)} \int_{\omega} \varphi^{(\ell)}\left(u^{+}-u^{-}\right) d x_{\alpha}+o(1),
\end{aligned}
$$

as $\gamma \rightarrow 0^{+}$, which completes the proof.

We now prove the liminf inequality for any arbitrary converging sequence.

Lemma 6.3. Let $\ell \in[0,+\infty]$. For every sequence $\left(u_{j}\right)$ converging to $\left(u^{+}, u^{-}\right)$we have

$$
\begin{aligned}
\liminf _{j \rightarrow+\infty} \mathcal{F}_{j}\left(u_{j}\right) \geq & \int_{\omega} \mathcal{Q}_{n-1} \bar{W}\left(D_{\alpha} u^{+}\right) d x_{\alpha}+\int_{\omega} \mathcal{Q}_{n-1} \bar{W}\left(D_{\alpha} u^{-}\right) d x_{\alpha} \\
& +R^{(\ell)} \int_{\omega} \varphi^{(\ell)}\left(u^{+}-u^{-}\right) d x_{\alpha} .
\end{aligned}
$$

Proof. Let $\left(u_{j}\right) \rightarrow\left(u^{+}, u^{-}\right)$be such that $\liminf _{j \rightarrow+\infty} \mathcal{F}_{j}\left(u_{j}\right)<+\infty$. Reasoning as in [2] Proposition 5.2 , by [11] Lemma 3.5, upon passing to a subsequence, for every $M>0$ and $\eta>0$, we have the existence of $R_{M}>M$ and of a Lipschitz function $\Phi_{M} \in \mathcal{C}_{c}^{1}\left(\mathbb{R}^{m} ; \mathbb{R}^{m}\right)$ with $\operatorname{Lip}\left(\Phi_{M}\right)=1$ such that

$$
\Phi_{M}(z)=\left\{\begin{array}{ccc}
z & \text { if } & |z|<R_{M} \\
0 & \text { if } & |z|>2 R_{M}
\end{array}\right.
$$

and

$$
\liminf _{j \rightarrow+\infty} \mathcal{F}_{j}\left(u_{j}\right) \geq \liminf _{j \rightarrow+\infty} \mathcal{F}_{j}\left(\Phi_{M}\left(u_{j}\right)\right)-\eta .
$$

Note that $\left(\Phi_{M}\left(u_{j}\right)\right) \subset W^{1, p}\left(\Omega_{j} ; \mathbb{R}^{m}\right) \cap L^{\infty}\left(\Omega_{j} ; \mathbb{R}^{m}\right), \sup _{j \in \mathbb{N}}\left\|\Phi_{M}\left(u_{j}\right)\right\|_{L^{\infty}\left(\Omega_{j} ; \mathbb{R}^{m}\right)}<R_{M}$ and it converges to $\left(\Phi_{M}\left(u^{+}\right), \Phi_{M}\left(u^{-}\right)\right)$as $j \rightarrow+\infty$. Hence, if we apply $(6.1)$, Propositions 6.1 and 6.2 to $\left(\Phi_{M}\left(u_{j}\right)\right)$ in place of $\left(u_{j}\right)$, letting $\gamma \rightarrow 0$ and $k \rightarrow+\infty$, we get that

$$
\begin{aligned}
\liminf _{j \rightarrow+\infty} \mathcal{F}_{j}\left(\Phi_{M}\left(u_{j}\right)\right) \geq & \int_{\omega} \mathcal{Q}_{n-1} \bar{W}\left(D_{\alpha} \Phi_{M}\left(u^{+}\right)\right) d x_{\alpha}+\int_{\omega} \mathcal{Q}_{n-1} \bar{W}\left(D_{\alpha} \Phi_{M}\left(u^{-}\right)\right) d x_{\alpha} \\
& +R^{(\ell)} \int_{\omega} \varphi^{(\ell)}\left(\Phi_{M}\left(u^{+}\right)-\Phi_{M}\left(u^{-}\right)\right) d x_{\alpha} .
\end{aligned}
$$

Moreover $\Phi_{M}\left(u^{ \pm}\right) \rightarrow u^{ \pm}$weakly in $W^{1, p}\left(\omega ; \mathbb{R}^{m}\right)$ as $M \rightarrow+\infty$; hence, by (6.7), (6.8), the lower semicontinuity of $\int_{\omega} \mathcal{Q}_{n-1} \bar{W}\left(D_{\alpha} u\right) d x_{\alpha}$ with respect to the weak $W^{1, p}\left(\omega ; \mathbb{R}^{m}\right)$-convergence, and (5.6) we have 
that

$$
\begin{aligned}
& \liminf _{j \rightarrow+\infty} \mathcal{F}_{j}\left(u_{j}\right) \\
\geq & \int_{\omega} \mathcal{Q}_{n-1} \bar{W}\left(D_{\alpha} u^{+}\right) d x_{\alpha}+\int_{\omega} \mathcal{Q}_{n-1} \bar{W}\left(D_{\alpha} u^{-}\right) d x_{\alpha}+R^{(\ell)} \int_{\omega} \varphi^{(\ell)}\left(u^{+}-u^{-}\right) d x_{\alpha}-\eta,
\end{aligned}
$$

and by the arbitrariness of $\eta$, the thesis.

6.2. The limsup inequality. For every $\left(u^{+}, u^{-}\right) \in W^{1, p}\left(\omega, \mathbb{R}^{m}\right) \times W^{1, p}\left(\omega, \mathbb{R}^{m}\right)$ the limsup inequality is obtained by suitably modifying the recovery sequences $\left(u_{j}^{ \pm}\right)$for the $\Gamma$-limits of

$$
\frac{1}{\delta_{j}} \int_{\omega^{+\delta_{j}}} W(D u) d x \quad \text { and } \quad \frac{1}{\delta_{j}} \int_{\omega^{-\delta_{j}}} W(D u) d x .
$$

Lemma 6.4. Let $\ell \in[0,+\infty]$ and let $\omega$ be an open bounded subset of $\mathbb{R}^{n-1}$ such that $\mathcal{H}^{n-1}(\partial \omega)=0$. Then, for all $\left(u^{+}, u^{-}\right) \in W^{1, p}\left(\omega, \mathbb{R}^{m}\right) \times W^{1, p}\left(\omega, \mathbb{R}^{m}\right)$ and for all $\eta>0$ there exists a sequence $\left(\bar{u}_{j}\right) \subset$ $W^{1, p}\left(\Omega_{j} ; \mathbb{R}^{m}\right)$ converging to $\left(u^{+}, u^{-}\right)$such that

$$
\begin{aligned}
\limsup _{j \rightarrow+\infty} \mathcal{F}_{j}\left(\bar{u}_{j}\right) \leq & \int_{\omega} \mathcal{Q}_{n-1} \bar{W}\left(D_{\alpha} u^{+}\right) d x_{\alpha}+\int_{\omega} \mathcal{Q}_{n-1} \bar{W}\left(D_{\alpha} u^{-}\right) d x_{\alpha} \\
& +R^{(\ell)} \int_{\omega} \varphi^{(\ell)}\left(u^{+}-u^{-}\right) d x_{\alpha}+\eta R^{(\ell)} \mathcal{H}^{n-1}(\omega) .
\end{aligned}
$$

Proof. The proof of the limsup is divided into three steps. We first construct a sequence $\left(\bar{u}_{j}\right) \subset$ $W^{1, p}\left(\Omega_{j} ; \mathbb{R}^{m}\right)$ that we expect to be a recovery sequence. In the second step we prove that $\left(\bar{u}_{j}\right)$ converges to $\left(u^{+}, u^{-}\right)$. Finally, we prove that it satisfies the limsup inequality. We first deal with the case $\ell \in(0,+\infty]$.

Step 1: Definition of a recovery sequence. Let $u^{ \pm} \in W^{1, p}\left(\omega ; \mathbb{R}^{m}\right) \cap L^{\infty}\left(\omega ; \mathbb{R}^{m}\right)$. According to [23] Theorem 2 and [8] Theorem 1.1, there exist two sequences $\left(u_{j}^{ \pm}\right) \subset W^{1, p}\left(\omega^{ \pm \delta_{j}} ; \mathbb{R}^{m}\right)$ such that $u_{j}^{ \pm} \rightarrow u^{ \pm}$, the sequences of gradients $\left(\left|D u_{j}^{ \pm}\right|^{p} / \delta_{j}\right)$ are equi-integrable on $\omega^{ \pm \delta_{j}}$, respectively, and

$$
\lim _{j \rightarrow+\infty} \frac{1}{\delta_{j}} \int_{\omega^{ \pm \delta_{j}}} W\left(D u_{j}^{ \pm}\right) d x=\int_{\omega} \mathcal{Q}_{n-1} \bar{W}\left(D_{\alpha} u^{ \pm}\right) d x_{\alpha} .
$$

Moreover, using a truncation argument (as in [3] Lemma 6.1, Step 2) we may assume without loss of generality that

$$
\sup _{j \in \mathbb{N}}\left\|u_{j}^{ \pm}\right\|_{L^{\infty}\left(\omega^{\left. \pm \delta_{j} ; \mathbb{R}^{m}\right)}\right.}<+\infty .
$$

Let $u_{j}:=u_{j}^{+} \chi_{\omega^{+\delta_{j}}}+u_{j}^{-} \chi_{\omega^{-\delta_{j}}} \in W^{1, p}\left(\omega^{+\delta_{j}} \cup \omega^{-\delta_{j}} ; \mathbb{R}^{m}\right)$ and let $\left(w_{j}\right)$ be the sequence obtained from $\left(u_{j}\right)$ as in Lemma 4.4, then $\sup _{j \in \mathbb{N}}\left\|w_{j}\right\|_{L^{\infty}\left(\omega^{\left. \pm \delta_{j} ; \mathbb{R}^{m}\right)}\right.}<+\infty$.

We first define $\left(\bar{u}_{j}\right)$ 'far' from the connecting zones; i.e.,

$$
\bar{u}_{j}:=w_{j} \text { in }\left(\omega \backslash \bigcup_{i \in \mathbb{Z}^{n-1}} B_{\rho_{j}}^{n-1}\left(x_{i}^{\varepsilon_{j}}\right)\right)^{ \pm \delta_{j}} .
$$

Then we pass to define $\left(\bar{u}_{j}\right)$ on each $B_{\rho_{j}}^{n-1}\left(x_{i}^{\varepsilon_{j}}\right)^{ \pm \delta_{j}}$ making a distinction between the indices $i \in Z_{j}$ and $i \in \mathbb{Z}^{n-1} \backslash Z_{j}$.

If $i \in Z_{j}$, by (5.2), for every $\eta>0$ there exists $\zeta_{\gamma, j}^{i} \in X_{j}^{\gamma}\left(u_{j}^{i+}-u_{j}^{i-}\right)$ such that

$$
\int_{\left(B_{\gamma N_{j}}^{n-1} \times I\right) \backslash C_{1, \gamma N_{j}}} r_{j}^{p} W\left(r_{j}^{-1} D_{\alpha} \zeta_{\gamma, j}^{i} \mid \delta_{j}^{-1} D_{n} \zeta_{\gamma, j}^{i}\right) d x \leq \varphi_{\gamma, j}^{(\ell)}\left(u_{j}^{i+}-u_{j}^{i-}\right)+\eta .
$$

Then, we define

$$
\bar{u}_{j}:=\zeta_{\gamma, j}^{i}\left(\frac{x_{\alpha}-x_{i}^{\varepsilon_{j}}}{r_{j}}, \frac{x_{n}}{\delta_{j}}\right)+u_{j}^{i-} \text { in } B_{\rho_{j}}^{n-1}\left(x_{i}^{\varepsilon_{j}}\right)^{ \pm \delta_{j}}, \quad i \in Z_{j} .
$$

In particular, $\bar{u}_{j}=u_{j}^{i \pm}=w_{j}$ on $\left(\partial B_{\rho_{j}}^{n-1}\left(x_{i}^{\varepsilon_{j}}\right)\right)^{ \pm \delta_{j}}$. 
Let us now deal with the contact zones not well contained in $\omega$; i.e., with the indices $i \notin Z_{j}$. For fixed $\gamma>0$ and $j$ large enough we have that $\gamma N_{j}>2$. Let $\psi \in W^{1, p}\left(B_{2}^{n-1} ;[0,1]\right)$ be such that $\psi=1$ on $\partial B_{2}^{n-1}$ and $\psi=0$ in $B_{1}^{n-1}$ and define

$$
\psi_{\gamma, j}(x):=\left\{\begin{array}{lll}
0 & \text { in } & \left(B_{\gamma N_{j}}^{n-1}\right)^{-} \\
\psi\left(x_{\alpha}\right) & \text { in } & \left(B_{2}^{n-1}\right)^{+} \\
1 & \text { in } & \left(B_{\gamma N_{j}}^{n-1} \backslash B_{2}^{n-1}\right)^{+} .
\end{array}\right.
$$

Then $\psi_{\gamma, j} \in W^{1, p}\left(\left(B_{\gamma N_{j}}^{n-1} \times I\right) \backslash C_{1, \gamma N_{j}} ;[0,1]\right), \psi_{\gamma, j}=1$ on $\left(\partial B_{\gamma N_{j}}^{n-1}\right)^{+}$and $\psi_{\gamma, j}=0$ on $\left(\partial B_{\gamma N_{j}}^{n-1}\right)^{-}$. Let $w_{j}^{ \pm}=w_{j} \chi_{\omega^{ \pm \delta_{j}}}$, we extend both of them to the whole $\omega \times\left(-\delta_{j}, \delta_{j}\right)$ by reflection; i.e., we define $\tilde{w}_{j}^{ \pm}\left(x_{\alpha}, x_{n}\right)=w_{j}^{ \pm}\left(x_{\alpha},-x_{n}\right)$ for $x \in \omega^{\mp \delta_{j}}$ and $\tilde{w}_{j}^{ \pm}(x)=w_{j}^{ \pm}(x)$ for $x \in \omega^{ \pm \delta_{j}}$. Hence, we define

$$
\bar{u}_{j}:=\psi_{\gamma, j}\left(\frac{x_{\alpha}-x_{i}^{\varepsilon_{j}}}{r_{j}}, \frac{x_{n}}{\delta_{j}}\right) \tilde{w}_{j}^{+}+\left(1-\psi_{\gamma, j}\left(\frac{x_{\alpha}-x_{i}^{\varepsilon_{j}}}{r_{j}}, \frac{x_{n}}{\delta_{j}}\right)\right) \tilde{w}_{j}^{-}
$$

in $\left(B_{\rho_{j}}^{n-1}\left(x_{i}^{\varepsilon_{j}}\right) \times\left(-\delta_{j}, \delta_{j}\right)\right) \cap \Omega_{j}$ and for $i \in \mathbb{Z}^{n-1} \backslash Z_{j}$. In particular, we have that $\bar{u}_{j}=w_{j}$ on $\left(\partial B_{\rho_{j}}^{n-1}\left(x_{i}^{\varepsilon_{j}}\right) \times\right.$ $\left.\left(-\delta_{j}, \delta_{j}\right)\right) \cap \Omega_{j}$; thus $\left(\bar{u}_{j}\right) \subset W^{1, p}\left(\Omega_{j} ; \mathbb{R}^{m}\right)$.

Step 2: The sequence $\left(\bar{u}_{j}\right)$ weakly converges to $\left(u^{+}, u^{-}\right)$. Let us check (3.1) and (3.2). We will only treat the upper cylinder $\omega^{+\delta_{j}}$, the lower part being analogous. First

$$
\begin{aligned}
& \frac{1}{\delta_{j}} \int_{\omega^{+\delta_{j}}}\left|\bar{u}_{j}-u^{+}\right|^{p} d x \\
& =\frac{1}{\delta_{j}} \int_{\left(\omega \backslash \bigcup_{i \in \mathbb{Z}^{n-1}} B_{\rho_{j}}^{n-1}\left(x_{i}^{\varepsilon_{j}}\right)\right)^{+\delta_{j}}}\left|w_{j}^{+}-u^{+}\right|^{p} d x \\
& +\frac{1}{\delta_{j}} \sum_{i \in Z_{j}} \int_{B_{\rho_{j}}^{n-1}\left(x_{i}^{\varepsilon_{j}}\right)^{+\delta_{j}}}\left|\zeta_{\gamma, j}^{i}\left(\frac{x_{\alpha}-x_{i}^{\varepsilon_{j}}}{r_{j}}, \frac{x_{n}}{\delta_{j}}\right)+u_{j}^{i-}-u^{+}\right|^{p} d x \\
& +\frac{1}{\delta_{j}} \sum_{i \in \mathbb{Z}^{n-1} \backslash Z_{j}} \int_{\left(\omega \cap B_{\rho_{j}}^{n-1}\left(x_{i}^{\varepsilon_{j}}\right)\right)}\left|\psi_{\gamma, j}\left(\frac{x_{\alpha}-x_{i}^{\varepsilon_{j}}}{r_{j}}, \frac{x_{n}}{\delta_{j}}\right)\left(w_{j}^{+}-\tilde{w}_{j}^{-}\right)+\tilde{w}_{j}^{-}-u^{+}\right|^{p} d x \\
& \leq \frac{1}{\delta_{j}} \int_{\omega^{+\delta_{j}}}\left|w_{j}-u^{+}\right|^{p} d x+c \sum_{i \in Z_{j}} \int_{B_{\rho_{j}}^{n-1}\left(x_{i}^{\varepsilon_{j}}\right)}\left|u^{+}-u_{j}^{i+}\right|^{p} d x_{\alpha} \\
& +\frac{c}{\delta_{j}} \sum_{i \in Z_{j}} \int_{B_{\rho_{j}}^{n-1}\left(x_{i}^{\varepsilon_{j}}\right)^{+\delta_{j}}}\left|\zeta_{\gamma, j}^{i}\left(\frac{x_{\alpha}-x_{i}^{\varepsilon_{j}}}{r_{j}}, \frac{x_{n}}{\delta_{j}}\right)-\left(u_{j}^{i+}-u_{j}^{i-}\right)\right|^{p} d x \\
& +\frac{c}{\delta_{j}} \int_{\left(\omega \cap \bigcup_{i \in \mathbb{Z}^{n-1} \backslash Z_{j}} B_{\rho_{j}}^{n-1}\left(x_{i}^{\varepsilon_{j}}\right)\right)^{+\delta_{j}}}\left(\left|w_{j}^{+}\right|^{p}+\left|\tilde{w}_{j}^{-}\right|^{p}+\left|u^{+}\right|^{p}\right) d x .
\end{aligned}
$$

Since $\lim _{j \rightarrow+\infty} \mathcal{H}^{n-1}\left(\omega \cap \bigcup_{i \in \mathbb{Z}^{n-1} \backslash Z_{j}} B_{\rho_{j}}^{n-1}\left(x_{i}^{\varepsilon_{j}}\right)\right)=0$ and $\sup _{j \in \mathbb{N}}\left\|w_{j}^{ \pm}\right\|_{L^{\infty}\left(\omega^{\left. \pm \delta_{j} ; \mathbb{R}^{m}\right)}\right.}<+\infty$, we have that

$$
\lim _{j \rightarrow+\infty} \frac{c}{\delta_{j}} \int_{\left(\omega \cap \bigcup_{i \in \mathbb{Z}^{n-1} \backslash Z_{j}} B_{\rho_{j}}^{n-1}\left(x_{i}^{\varepsilon_{j}}\right)\right)}\left(\left|w_{j}^{+}\right|^{p}+\left|\tilde{w}_{j}^{-}\right|^{p}+\left|u^{+}\right|^{p}\right) d x=0 .
$$

Moreover, reasoning as in the proof of Proposition 5.3 (see inequality (5.24)), we have that

$$
\lim _{j \rightarrow+\infty} \sum_{i \in Z_{j}} \int_{B_{\rho_{j}}^{n-1}\left(x_{i}^{\varepsilon_{j}}\right)}\left|u^{+}-u_{j}^{i+}\right|^{p} d x_{\alpha}=0,
$$

and, by the convergence $w_{j} \rightarrow\left(u^{+}, u^{-}\right)$, it remains only to prove that

$$
\lim _{j \rightarrow+\infty} \frac{1}{\delta_{j}} \sum_{i \in Z_{j}} \int_{B_{\rho_{j}}^{n-1}\left(x_{i}^{\varepsilon_{j}}\right)^{+\delta_{j}}}\left|\zeta_{\gamma, j}^{i}\left(\frac{x_{\alpha}-x_{i}^{\varepsilon_{j}}}{r_{j}}, \frac{x_{n}}{\delta_{j}}\right)-\left(u_{j}^{i+}-u_{j}^{i-}\right)\right|^{p} d x=0 .
$$


In fact, changing variable, we get that

$$
\begin{aligned}
& \frac{1}{\delta_{j}} \sum_{i \in Z_{j}} \int_{B_{\rho_{j}}^{n-1}\left(x_{i}^{\varepsilon_{j}}\right)+\delta_{j}}\left|\zeta_{\gamma, j}^{i}\left(\frac{x_{\alpha}-x_{i}^{\varepsilon_{j}}}{r_{j}}, \frac{x_{n}}{\delta_{j}}\right)-\left(u_{j}^{i+}-u_{j}^{i-}\right)\right|^{p} d x \\
= & r_{j}^{n-1} \sum_{i \in Z_{j}} \int_{\left(B_{\gamma N_{j}}^{n-1}\right)+}\left|\zeta_{\gamma, j}^{i}(x)-\left(u_{j}^{i+}-u_{j}^{i-}\right)\right|^{p} d x,
\end{aligned}
$$

and by, Poincaré's Inequality

$$
\int_{B_{\gamma N_{j}}^{n-1}}\left|\zeta_{\gamma, j}^{i}\left(x_{\alpha}, x_{n}\right)-\left(u_{j}^{i+}-u_{j}^{i-}\right)\right|^{p} d x_{\alpha} \leq c\left(\gamma N_{j}\right)^{p} \int_{B_{\gamma N_{j}}^{n-1}}\left|D_{\alpha} \zeta_{\gamma, j}^{i}\left(x_{\alpha}, x_{n}\right)\right|^{p} d x_{\alpha}
$$

for a.e. $x_{n} \in(0,1)$. Hence, by the $p$-growth condition (3.3) and (6.12) if we integrate with respect to $x_{n}$ and sum up in $i \in Z_{j}$, we get that

$$
\begin{aligned}
& \frac{1}{\delta_{j}} \sum_{i \in Z_{j}} \int_{B_{\rho_{j}}^{n-1}\left(x_{i}^{\varepsilon_{j}}\right)^{+\delta_{j}}}\left|\zeta_{\gamma, j}^{i}\left(\frac{x_{\alpha}-x_{i}^{\varepsilon_{j}}}{r_{j}}, \frac{x_{n}}{\delta_{j}}\right)-\left(u_{j}^{i+}-u_{j}^{i-}\right)\right|^{p} d x \\
\leq & c r_{j}^{n-1} \gamma^{p} N_{j}^{p} \sum_{i \in Z_{j}} \int_{\left(B_{\gamma N_{j}}^{n-1}\right)^{+}}\left|D_{\alpha} \zeta_{\gamma, j}^{i}\right|^{p} d x \\
\leq & c r_{j}^{n-1} \gamma^{p} N_{j}^{p} \sum_{i \in Z_{j}} \int_{\left(B_{\gamma N_{j}}^{n-1}\right)^{+}}\left|\left(D_{\alpha} \zeta_{\gamma, j}^{i} \mid \frac{r_{j}}{\delta_{j}} D_{n} \zeta_{\gamma, j}^{i}\right)\right|^{p} d x \\
\leq & c r_{j}^{n-1} \gamma^{p} N_{j}^{p} \sum_{i \in Z_{j}}\left(\varphi_{\gamma, j}^{(\ell)}\left(u_{j}^{i+}-u_{j}^{i-}\right)+\eta+r_{j}^{p} \mathcal{H}^{n-1}\left(B_{\gamma N_{j}}^{n-1}\right)\right) \\
\leq & c \gamma^{p} \varepsilon_{j}^{p} \frac{r_{j}^{n-1-p}}{\varepsilon_{j}^{n-1}}\left(\sum_{i \in Z_{j}} \varepsilon_{j}^{n-1} \varphi_{\gamma, j}^{(\ell)}\left(u_{j}^{i+}-u_{j}^{i-}\right)+\left(\eta+c \gamma^{n-1} \frac{\varepsilon_{j}^{n-1}}{r_{j}^{n-1-p}}\right) \mathcal{H}^{n-1}(\omega)\right)
\end{aligned}
$$

By Proposition 5.3 and (5.3), passing to the limit as $j \rightarrow+\infty$ in (6.19), we get (6.18).

It remains to prove that $(3.2)$ holds. In fact,

$$
\begin{aligned}
& \frac{1}{\delta_{j}} \int_{\omega^{+\delta_{j}}}\left|D \bar{u}_{j}\right|^{p} d x \\
= & \frac{1}{\delta_{j}} \int_{\left(\omega \backslash \bigcup_{i \in \mathbb{Z}^{n-1}} B_{\rho_{j}}^{n-1}\left(x_{i}^{\varepsilon_{j}}\right)\right)^{+\delta_{j}}}\left|D w_{j}^{ \pm}\right|^{p} d x \\
& +\frac{1}{\delta_{j}} \int_{\bigcup_{i \in Z_{j}} B_{\rho_{j}}^{n-1}\left(x_{i}^{\varepsilon_{j}}\right)^{+\delta_{j}} \mid}\left|\left(r_{j}^{-1} D_{\alpha} \zeta_{\gamma, j}^{i}\left(\frac{x_{\alpha}-x_{i}^{\varepsilon_{j}}}{r_{j}}, \frac{x_{n}}{\delta_{j}}\right) \mid \delta_{j}^{-1} D_{n} \zeta_{\gamma, j}^{i}\left(\frac{x_{\alpha}-x_{i}^{\varepsilon_{j}}}{r_{j}}, \frac{x_{n}}{\delta_{j}}\right)\right)\right|^{p} d x \\
& +\frac{1}{\delta_{j}} \int_{\left(\bigcup_{i \in \mathbb{Z}^{n-1} \backslash Z_{j}} B_{\rho_{j}}^{n-1}\left(x_{i}^{\varepsilon_{j}}\right) \cap \omega\right)^{+\delta_{j}}}\left|D \bar{u}_{j}\right|^{p} d x .
\end{aligned}
$$

It can be easily shown that

$$
\begin{aligned}
& \frac{1}{\delta_{j}} \int_{\bigcup_{i \in Z_{j}} B_{\rho_{j}}^{n-1}\left(x_{i}^{\varepsilon_{j}}\right)^{+\delta_{j}}}\left|\left(r_{j}^{-1} D_{\alpha} \zeta_{\gamma, j}^{i}\left(\frac{x_{\alpha}-x_{i}^{\varepsilon_{j}}}{r_{j}}, \frac{x_{n}}{\delta_{j}}\right) \mid \delta_{j}^{-1} D_{n} \zeta_{\gamma, j}^{i}\left(\frac{x_{\alpha}-x_{i}^{\varepsilon_{j}}}{r_{j}}, \frac{x_{n}}{\delta_{j}}\right)\right)\right|^{p} d x \\
\leq & \frac{r_{j}^{n-1-p}}{\varepsilon_{j}^{n-1}}\left(\sum_{i \in Z_{j}} \varepsilon_{j}^{n-1} \varphi_{\gamma, j}^{(\ell)}\left(u_{j}^{i+}-u_{j}^{i-}\right)\right)+\mathcal{H}^{n-1}(\omega)\left(\eta \frac{r_{j}^{n-1-p}}{\varepsilon_{j}^{n-1}}+\gamma^{n-1}\right)
\end{aligned}
$$


while,

$$
\begin{aligned}
& \frac{1}{\delta_{j}} \int_{\left(\cup_{i \in \mathbb{Z}^{n-1} \backslash Z_{j}} B_{\rho_{j}}^{n-1}\left(x_{i}^{\varepsilon_{j}}\right) \cap \omega\right)^{+\delta_{j}}}\left|D \bar{u}_{j}\right|^{p} d x \\
& \leq c \sum_{i \in \mathbb{Z}^{n-1} \backslash Z_{j}}\left(\frac{1}{r_{j}^{p} \delta_{j}} \int_{\left(B_{\rho_{j}}^{n-1}\left(x_{i}^{\varepsilon_{j}}\right) \cap \omega\right)^{+\delta_{j}}}\left|D_{\alpha} \psi_{\gamma, j}\left(\frac{x_{\alpha}-x_{i}^{\varepsilon_{j}}}{r_{j}}, \frac{x_{n}}{\delta_{j}}\right)\right|^{p}\left(\left|w_{j}^{+}\right|^{p}+\left|\tilde{w}_{j}^{-}\right|^{p}\right) d x\right. \\
& \left.+\frac{1}{\delta_{j}} \int_{\left(B_{\rho_{j}}^{n-1}\left(x_{i}^{\varepsilon_{j}}\right) \cap \omega\right)^{+\delta_{j}}}\left(\left|D w_{j}^{+}\right|^{p}+\left|D \tilde{w}_{j}^{-}\right|^{p}\right) d x\right) \\
& \leq c \sum_{i \in \mathbb{Z}^{n-1} \backslash Z_{j}}\left(r_{j}^{n-1-p} \int_{B_{2}^{n-1}}\left|D_{\alpha} \psi\right|^{p} d x_{\alpha}+\frac{1}{\delta_{j}} \int_{\left(B_{\rho_{j}}^{n-1}\left(x_{i}^{\varepsilon_{j}}\right) \cap \omega\right)^{+\delta_{j}}}\left|D w_{j}^{+}\right|^{p} d x\right. \\
& \left.+\frac{1}{\delta_{j}} \int_{\left(B_{\rho_{j}}^{n-1}\left(x_{i}^{\varepsilon_{j}}\right) \cap \omega\right)^{-\delta_{j}}}\left|D w_{j}^{-}\right|^{p} d x\right) \\
& \leq c \sum_{i \in \mathbb{Z}^{n-1} \backslash Z_{j}}\left(\frac{r_{j}^{n-1-p}}{\varepsilon_{j}^{n-1}} \mathcal{H}^{n-1}\left(Q_{i, \varepsilon_{j}}^{n-1}\right)+\frac{1}{\delta_{j}} \int_{\left(B_{\rho_{j}}^{n-1}\left(x_{i}^{\varepsilon_{j}}\right) \cap \omega\right)^{ \pm \delta_{j}}}\left|D w_{j}^{ \pm}\right|^{p} d x\right. \\
& \left.+\frac{1}{\delta_{j}} \int_{\left(B_{\rho_{j}}^{n-1}\left(x_{i}^{\varepsilon_{j}}\right) \cap \omega\right)^{-\delta_{j}}}\left|D w_{j}^{-}\right|^{p} d x\right) .
\end{aligned}
$$

Note that the previous sum can be computed over all $i \in \mathbb{Z}^{n-1} \backslash Z_{j}$ such that $Q_{i, \varepsilon_{j}}^{n-1} \cap \omega \neq \emptyset$. Let

$$
\omega_{j}^{\prime}:=\bigcup_{i \in \mathbb{Z}^{n-1} \backslash Z_{j}, Q_{i, \varepsilon_{j}}^{n-1} \cap \omega \neq \emptyset} Q_{i, \varepsilon_{j}}^{n-1},
$$

then

$$
\sum_{i \in \mathbb{Z}^{n-1} \backslash Z_{j}, Q_{i, \varepsilon_{j}}^{n-1} \cap \omega \neq \emptyset} \mathcal{H}^{n-1}\left(Q_{i, \varepsilon_{j}}^{n-1}\right)=\mathcal{H}^{n-1}\left(\omega_{j}^{\prime}\right) \rightarrow \mathcal{H}^{n-1}(\partial \omega)=0 .
$$

Moreover, by Lemma 4.4 we have that $\sup _{j} \frac{1}{\delta_{j}} \int_{\omega^{ \pm \delta_{j}}}\left|D w_{j}^{ \pm}\right|^{p} d x<+\infty$; hence, by Proposition 5.3, (5.3), (6.20), (6.21) and (6.22) we get (3.2).

Step 3: The sequence $\left(\bar{u}_{j}\right)$ is a recovery sequence. We now prove the limsup inequality.

$$
\begin{aligned}
& \limsup _{j \rightarrow+\infty} \int_{\omega^{ \pm \delta_{j}}} W\left(D \bar{u}_{j}\right) d x \\
&=\limsup _{j \rightarrow+\infty} \frac{1}{\delta_{j}}\left(\int_{\left(\omega \backslash \bigcup_{i \in \mathbb{Z}^{n-1}} B_{\rho_{j}}^{n-1}\left(x_{i}^{\varepsilon_{j}}\right)\right)^{ \pm \delta_{j}}} W\left(D \bar{u}_{j}\right) d x+\int_{\bigcup_{i \in Z_{j}} B_{\rho_{j}}^{n-1}\left(x_{i}^{\varepsilon_{j}}\right)^{ \pm \delta_{j}}} W\left(D \bar{u}_{j}\right) d x\right. \\
&\left.\quad+\int_{\left(\omega \cap \bigcup_{i \in \mathbb{Z}^{n-1} \backslash Z_{j}} B_{\rho_{j}}^{n-1}\left(x_{i}^{\varepsilon_{j}}\right)\right)^{ \pm \delta_{j}}} W\left(D \bar{u}_{j}\right) d x\right) .
\end{aligned}
$$


We deal with the first term in (6.24). The definition of $\bar{u}_{j}(6.11)$, Lemma 4.4 and (6.10), yield

$$
\begin{aligned}
& \limsup _{j \rightarrow+\infty} \frac{1}{\delta_{j}} \int_{\left(\omega \backslash \bigcup_{i \in \mathbb{Z}^{n-1}} B_{\rho_{j}}^{n-1}\left(x_{i}^{\varepsilon_{j}}\right)\right)^{ \pm \delta_{j}}} W\left(D \bar{u}_{j}\right) d x \\
= & \limsup _{j \rightarrow+\infty} \frac{1}{\delta_{j}} \int_{\left(\omega \backslash \bigcup_{i \in \mathbb{Z}^{n-1}} B_{\rho_{j}}^{n-1}\left(x_{i}^{\varepsilon_{j}}\right)\right)^{ \pm \delta_{j}}} W\left(D w_{j}\right) d x \\
\leq & \limsup _{j \rightarrow+\infty} \frac{1}{\delta_{j}} \int_{\omega^{ \pm \delta_{j}}} W\left(D u_{j}^{ \pm}\right) d x+o(1) \\
= & \int_{\omega} \mathcal{Q}_{n-1} \bar{W}\left(D_{\alpha} u^{ \pm}\right) d x_{\alpha}+o(1),
\end{aligned}
$$

as $\gamma \rightarrow 0^{+}$. For every $i \in Z_{j}$, by (6.13) and (6.12) we get that

$$
\begin{aligned}
& \frac{1}{\delta_{j}}\left(\int_{B_{\rho_{j}}^{n-1}\left(x_{i}^{\varepsilon_{j}}\right)^{+\delta_{j}}} W\left(D \bar{u}_{j}\right) d x+\int_{B_{\rho_{j}}^{n-1}\left(x_{i}^{\varepsilon_{j}}\right)^{-\delta_{j}}} W\left(D \bar{u}_{j}\right) d x\right) \\
= & r_{j}^{n-1} \int_{\left(B_{\gamma N_{j}}^{n-1} \times I\right) \backslash C_{1, \gamma N_{j}}} W\left(r_{j}^{-1} D_{\alpha} \zeta_{\gamma, j}^{i} \mid \delta_{j}^{-1} D_{n} \zeta_{\gamma, j}^{i}\right) d x \\
\leq & r_{j}^{n-1-p}\left(\varphi_{\gamma, j}^{(\ell)}\left(u_{j}^{i+}-u_{j}^{i-}\right)+\eta\right) ;
\end{aligned}
$$

hence, by (5.3) and Proposition 5.3 we get

$$
\begin{aligned}
& \limsup _{j \rightarrow+\infty} \frac{1}{\delta_{j}}\left(\int_{\bigcup_{i \in Z_{j}} B_{\rho_{j}}^{n-1}\left(x_{i}^{\varepsilon_{j}}\right)^{+\delta_{j}}} W\left(D \bar{u}_{j}\right) d x+\int_{\bigcup_{i \in Z_{j}} B_{\rho_{j}}^{n-1}\left(x_{i}^{\varepsilon_{j}}\right)^{-\delta_{j}}} W\left(D \bar{u}_{j}\right) d x\right) \\
\leq \quad & R^{(\ell)} \int_{\omega} \varphi^{(\ell)}\left(u^{+}-u^{-}\right) d x_{\alpha}+R^{(\ell)} \mathcal{H}^{n-1}(\omega) \eta \\
& +\limsup _{j \rightarrow+\infty} \int_{\omega}\left|\sum_{i \in Z_{j}} \varphi_{\gamma, j}^{(\ell)}\left(u_{j}^{i+}-u_{j}^{i-}\right) \chi_{Q_{i, \varepsilon_{j}}^{n-1}}-\varphi^{(\ell)}\left(u^{+}-u^{-}\right)\right| d x_{\alpha} \\
= & R^{(\ell)} \int_{\omega} \varphi^{(\ell)}\left(u^{+}-u^{-}\right) d x_{\alpha}+R^{(\ell)} \mathcal{H}^{n-1}(\omega) \eta+o(1),
\end{aligned}
$$

as $\gamma \rightarrow 0^{+}$. Finally, for $i \notin Z_{j}$, by the $p$-growth condition (3.3) and (6.22), we obtain

$$
\begin{aligned}
& \frac{1}{\delta_{j}}\left(\int_{\left(\bigcup_{i \in \mathbb{Z}^{n-1} \backslash Z_{j}} B_{\rho_{j}}^{n-1}\left(x_{i}^{\varepsilon_{j}}\right) \cap \omega\right)^{ \pm \delta_{j}}} W\left(D \bar{u}_{j}\right) d x\right) \\
\leq & \sum_{i \in \mathbb{Z}^{n-1} \backslash Z_{j}} \frac{\beta}{\delta_{j}}\left(\int_{\left(B_{\rho_{j}}^{n-1}\left(x_{i}^{\varepsilon_{j}}\right) \cap \omega\right)^{ \pm \delta_{j}}}\left(1+\left|D \bar{u}_{j}\right|^{p}\right) d x\right) \\
\leq & c \mathcal{H}^{n-1}\left(\bigcup_{i \in \mathbb{Z}^{n-1} \backslash Z_{j}} B_{\rho_{j}}^{n-1}\left(x_{i}^{\varepsilon_{j}}\right) \cap \omega\right) \\
& +c \sum_{i \in \mathbb{Z}^{n-1} \backslash Z_{j}}\left(\frac{r_{j}^{n-1-p}}{\varepsilon_{j}^{n-1}} \mathcal{H}^{n-1}\left(Q_{i, \varepsilon_{j}}^{n-1}\right)+\frac{1}{\delta_{j}} \int_{\left(B_{\rho_{j}}^{n-1}\left(x_{i}^{\varepsilon_{j}}\right) \cap \omega\right)^{+\delta_{j}}}\left|D w_{j}^{+}\right|^{p} d x\right. \\
& \left.+\frac{1}{\delta_{j}} \int_{\left(B_{\rho_{j}}^{n-1}\left(x_{i}^{\varepsilon_{j}}\right) \cap \omega\right)^{-\delta_{j}}}\left|D w_{j}^{-}\right|^{p} d x\right) .
\end{aligned}
$$

Since

$$
\lim _{j \rightarrow+\infty} \mathcal{H}^{n-1}\left(\bigcup_{i \in \mathbb{Z}^{n-1} \backslash Z_{j}} B_{\rho_{j}}^{n-1}\left(x_{i}^{\varepsilon_{j}}\right) \cap \omega\right)=0
$$


by (5.3), the equi-integrability of $\left(\left|D w_{j}^{ \pm}\right|^{p} / \delta_{j}\right)$ on $\omega^{ \pm \delta_{j}}$ and (6.23), we deduce

$$
\limsup _{j \rightarrow+\infty} \frac{1}{\delta_{j}} \int_{\left(\omega \cap \bigcup_{i \in \mathbb{Z}^{n-1} \backslash Z_{j}} B_{\rho_{j}}^{n-1}\left(x_{i}^{\varepsilon_{j}}\right)\right)^{ \pm \delta_{j}}} W\left(D \bar{u}_{j}\right) d x=0 .
$$

Gathering (6.24)-(6.27) and passing to the limit as $\gamma \rightarrow 0^{+}$we get the limsup inequality for every $u^{ \pm} \in W^{1, p}\left(\omega ; \mathbb{R}^{m}\right) \cap L^{\infty}\left(\omega ; \mathbb{R}^{m}\right)$.

We remove the boundedness assumption simply noting that any arbitrary $W^{1, p}\left(\omega ; \mathbb{R}^{m}\right)$ function can approximated by a sequence of functions belonging to $W^{1, p}\left(\omega ; \mathbb{R}^{m}\right) \cap L^{\infty}\left(\omega ; \mathbb{R}^{m}\right)$, with respect to the strong $W^{1, p}\left(\omega ; \mathbb{R}^{m}\right)$-convergence. Then, by the lower semicontinuity of the $\Gamma$-limsup and the continuity of

$$
\left(v^{+}, v^{-}\right) \mapsto \int_{\omega} \mathcal{Q}_{n-1} \bar{W}\left(D_{\alpha} v^{+}\right) d x_{\alpha}+\int_{\omega} \mathcal{Q}_{n-1} \bar{W}\left(D_{\alpha} v^{-}\right) d x_{\alpha}+R^{(\ell)} \int_{\omega} \varphi^{(\ell)}\left(v^{+}-v^{-}\right) d x_{\alpha}
$$

with respect to the strong $W^{1, p}\left(\omega ; \mathbb{R}^{m}\right)$-convergence we get the thesis for $\ell \in(0,+\infty]$.

If $\ell=0$, we can follow the line of the previous case with slight changes. Let us start by dealing with Step 1. First, we have to notice that for the definition of $\left(\bar{u}_{j}\right)$ in $B_{\rho_{j}}^{n-1}\left(x_{i}^{\varepsilon_{j}}\right)^{ \pm \delta_{j}}$, for $i \in Z_{j}$, we have to consider, for any $\eta>0$, a function $\zeta_{\gamma, j} \in Y_{j}^{\gamma}(z)$ such that

$$
\int_{\left(B_{\gamma N_{j}}^{n-1} \times I_{j}\right) \backslash C_{1, \gamma N_{j}}} r_{j}^{p} W\left(r_{j}^{-1} D \zeta_{\gamma, j}\right) d x \leq \varphi_{\gamma, j}^{(0)}(z)+\eta ;
$$

hence,

$$
\bar{u}_{j}\left(x_{\alpha}, x_{n}\right):=\zeta_{\gamma, j}^{i}\left(\frac{x_{\alpha}-x_{i}^{\varepsilon_{j}}}{r_{j}}, \frac{x_{n}}{r_{j}}\right)+u_{j}^{i-} \text { in } B_{\rho_{j}}^{n-1}\left(x_{i}^{\varepsilon_{j}}\right)^{ \pm \delta_{j}}, \quad \text { for } i \in Z_{j} .
$$

While for the definition of $\left(\bar{u}_{j}\right)$ in $B_{\rho_{j}}^{n-1}\left(x_{i}^{\varepsilon_{j}}\right)^{ \pm \delta_{j}}$, for $i \in \mathbb{Z}^{n-1} \backslash Z_{j}$, we have to introduce a suitable function $\psi_{\gamma, j}$ different from the one used in (6.14). In fact, for a fixed $\gamma>0$ and $j$ large enough we can always assume that $\gamma N_{j}>2$ and $\delta_{j} / r_{j}>2$. Let $\psi \in W^{1, p}\left(B_{2}^{n-1} \times(0,2) ;[0,1]\right)$ such that $\psi=0$ on $B_{1}^{n-1} \times\{0\}$ and $\psi=1$ on $\partial B_{2}^{n-1} \times(0,2)$. We then define

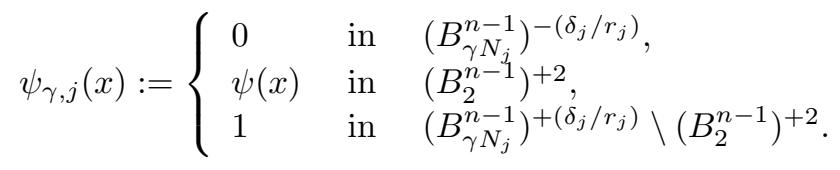

The functions $\psi_{\gamma, j}$ belong to $W^{1, p}\left(\left(B_{\gamma N_{j}}^{n-1} \times I_{j}\right) \backslash C_{1, \gamma N_{j}} ;[0,1]\right)$ and satisfy $\psi_{\gamma, j}=1$ on $\left(\partial B_{\gamma N_{j}}^{n-1}\right)^{+\left(\delta_{j} / r_{j}\right)}$ and $\psi_{\gamma, j}=0$ in $\left(B_{\gamma N_{j}}^{n-1}\right)^{-\left(\delta_{j} / r_{j}\right)}$. Hence, we define

$$
\bar{u}_{j}:=\psi_{\gamma, j}\left(\frac{x_{\alpha}-x_{i}^{\varepsilon_{j}}}{r_{j}}, \frac{x_{n}}{r_{j}}\right) \tilde{w}_{j}^{+}+\left(1-\psi_{\gamma, j}\left(\frac{x_{\alpha}-x_{i}^{\varepsilon_{j}}}{r_{j}}, \frac{x_{n}}{r_{j}}\right)\right) \tilde{w}_{j}^{-}
$$

in $\left(B_{\rho_{j}}^{n-1}\left(x_{i}^{\varepsilon_{j}}\right) \times\left(-\delta_{j}, \delta_{j}\right)\right) \cap \Omega_{j}$ and for $i \in \mathbb{Z}^{n-1} \backslash Z_{j}$. In particular, we have that $\bar{u}_{j}=w_{j}$ on $\left(\partial B_{\rho_{j}}^{n-1}\left(x_{i}^{\varepsilon_{j}}\right) \times\right.$ $\left.\left(-\delta_{j}, \delta_{j}\right)\right) \cap \Omega_{j}$.

Taking into account the definition of $\left(\bar{u}_{j}\right)$ we can proceed as in Steps 2 and 3 also for $\ell=0$.

\section{REPRESENTATION FORMUla FOR THE INTERFACIAL ENERGy DENSITY}

This section is devoted to describe explicitly the interfacial energy density $\varphi^{(\ell)}$ for $\ell \in[0,+\infty]$. As in [2], we expect to find a capacitary type formula for each regime $\ell \in(0,+\infty), \ell=+\infty$ and $\ell=0$.

We recall that $\varphi^{(\ell)}$ is the pointwise limit of the sequence $\left(\varphi_{\gamma, j}^{(\ell)}\right)$, as $j \rightarrow+\infty$ and $\gamma \rightarrow 0^{+}$where for $\ell \in(0,+\infty]$

$$
\varphi_{\gamma, j}^{(\ell)}(z)=\inf \left\{\int_{\left(B_{\gamma N_{j}}^{n-1} \times I\right) \backslash C_{1, \gamma N_{j}}} r_{j}^{p} W\left(r_{j}^{-1}\left(D_{\alpha} \zeta \mid \frac{r_{j}}{\delta_{j}} D_{n} \zeta\right)\right) d x: \quad \zeta \in X_{j}^{\gamma}(z)\right\},
$$


while for $\ell=0$,

$$
\varphi_{\gamma, j}^{(0)}(z)=\inf \left\{\int_{\left(B_{\gamma N_{j}}^{n-1} \times I_{j}\right) \backslash C_{1, \gamma N_{j}}} r_{j}^{p} W\left(r_{j}^{-1} D \zeta\right) d x: \quad \zeta \in Y_{j}^{\gamma}(z)\right\}
$$

(see Section 5). The main difficulty occurring in the description of $\varphi^{(\ell)}$ is due to the fact that the above minimum problems are stated on (increasingly) varying domains. This do not permit, for example, to deal with a direct $\Gamma$-convergence approach in order to apply the classical result on the convergence of associated minimum problems. Thus the proof of the representation formula will be performed in three main steps: we first prove an auxiliary $\Gamma$-convergence result for a suitable sequence of energies stated on a fixed domain, then we describe the functional space occurring in the limit capacitary formula, finally, we prove that $\varphi^{(\ell)}$ is described by a representation formula of capacitary-type.

We introduce some convenient notation for the sequel. Let $g_{j}: \mathbb{R}^{m \times n} \rightarrow[0,+\infty)$ be the sequence of functions given by

$$
g_{j}(F):=r_{j}^{p} W\left(r_{j}^{-1} F\right)
$$

for every $F \in \mathbb{R}^{m \times n}$. By (3.3) and (3.4) it follows that

$$
|F|^{p}-r_{j}^{p} \leq g_{j}(F) \leq \beta\left(r_{j}^{p}+|F|^{p}\right), \quad \text { for all } F \in \mathbb{R}^{m \times n}
$$

and the following $p$-Lipschitz condition holds:

$$
\left|g_{j}\left(F_{1}\right)-g_{j}\left(F_{2}\right)\right| \leq c\left(r_{j}^{p-1}+\left|F_{1}\right|^{p-1}+\left|F_{2}\right|^{p-1}\right)\left|F_{1}-F_{2}\right|, \quad \text { for all } F_{1}, F_{2} \in \mathbb{R}^{m \times n} .
$$

Then, according to Ascoli-Arzela's Theorem, up to subsequences, $g_{j}$ converges locally uniformly in $\mathbb{R}^{m \times n}$ to a function $g$ satisfying:

$$
|F|^{p} \leq g(F) \leq \beta|F|^{p}, \quad \text { for all } F \in \mathbb{R}^{m \times n}
$$

and

$$
\left|g\left(F_{1}\right)-g\left(F_{2}\right)\right| \leq c\left(\left|F_{1}\right|^{p-1}+\left|F_{2}\right|^{p-1}\right)\left|F_{1}-F_{2}\right|, \quad \text { for all } F_{1}, F_{2} \in \mathbb{R}^{m \times n} .
$$

7.1. The case $\ell \in(0,+\infty)$. We define

$$
\begin{aligned}
X_{N}(z):=\left\{\zeta \in W^{1, p}\left(\left(B_{N}^{n-1} \times I\right) \backslash C_{1, N} ; \mathbb{R}^{m}\right):\right. & \zeta=z \text { on }\left(\partial B_{N}^{n-1}\right)^{+} \\
& \text {and } \left.\zeta=0 \text { on }\left(\partial B_{N}^{n-1}\right)^{-}\right\}
\end{aligned}
$$

for $N>1$ and $I=(-1,1)$. We recall the following $\Gamma$-convergence result.

Proposition 7.1. Let

$$
\ell=\lim _{j \rightarrow+\infty} \frac{r_{j}}{\delta_{j}} \in(0,+\infty)
$$

then the sequence of functionals $G_{j}^{(\ell)}: L^{p}\left(\left(B_{N}^{n-1} \times I\right) \backslash C_{1, N} ; \mathbb{R}^{m}\right) \rightarrow[0,+\infty]$, defined by

$$
G_{j}^{(\ell)}(\zeta):= \begin{cases}\int_{\left(B_{N}^{n-1} \times I\right) \backslash C_{1, N}} g_{j}\left(D_{\alpha} \zeta \mid \frac{r_{j}}{\delta_{j}} D_{n} \zeta\right) d x & \text { if } \zeta \in X_{N}(z) \\ +\infty & \text { otherwise, }\end{cases}
$$

$\Gamma$-converges, with respect to the $L^{p}$-convergence, to

$$
G^{(\ell)}(\zeta):= \begin{cases}\int_{\left(B_{N}^{n-1} \times I\right) \backslash C_{1, N}} g\left(D_{\alpha} \zeta \mid \ell D_{n} \zeta\right) d x & \text { if } \zeta \in X_{N}(z) \\ +\infty & \text { otherwise. }\end{cases}
$$

Proof. Since $\ell=\lim _{j \rightarrow+\infty}\left(r_{j} / \delta_{j}\right) \in(0,+\infty)$, by the locally uniform convergence of $g_{j}$ to $g$ we have that the sequence of quasiconvex functions $F \mapsto g_{j}\left(\bar{F} \mid\left(r_{j} / \delta_{j}\right) F_{n}\right)$ pointwise converges to $F \mapsto g\left(\bar{F} \mid \ell F_{n}\right)$. Hence the conclusion comes from [10] Propositions 12.8 and 11.7. 
Remark 7.2. We denote by $p^{*}$ the Sobolev exponent in dimension $(n-1)$ i.e.

$$
p^{*}:=\frac{(n-1) p}{n-1-p} .
$$

We recall that if $(a, b) \subset \mathbb{R}$, the space $L^{p}\left(a, b ; L^{p^{*}}\left(\mathbb{R}^{n-1} ; \mathbb{R}^{m}\right)\right)$ is a reflexive and separable Banach space (see e.g. [1] or [30]). Hence, by the Banach-Alaoglu-Bourbaki Theorem, any bounded sequence admits a weakly converging subsequence.

Proposition 7.3 (Limit space). Let

$$
\ell=\lim _{j \rightarrow+\infty} \frac{r_{j}}{\delta_{j}} \in(0,+\infty), \quad 0<R^{(\ell)}=\lim _{j \rightarrow+\infty} \frac{r_{j}^{n-1-p}}{\varepsilon_{j}^{n-1}}<+\infty
$$

and let $\left(\zeta_{\gamma, j}\right) \in X_{j}^{\gamma}(z)$ such that, for every fixed $\gamma>0$,

$$
\sup _{j \in \mathbb{N}} \int_{\left(B_{\gamma N_{j}}^{n-1} \times I\right) \backslash C_{1, \gamma N_{j}}} g_{j}\left(D_{\alpha} \zeta_{\gamma, j} \mid \frac{r_{j}}{\delta_{j}} D_{n} \zeta_{\gamma, j}\right) d x \leq c .
$$

Then, there exists a sequence $\tilde{\zeta}_{j} \in W_{\mathrm{loc}}^{1, p}\left(\left(\mathbb{R}^{n-1} \times I\right) \backslash C_{1, \infty} ; \mathbb{R}^{m}\right)$ such that

$$
\tilde{\zeta}_{j}=\zeta_{\gamma, j} \quad \text { in } \quad\left(B_{\gamma N_{j}}^{n-1} \times I\right) \backslash C_{1, \gamma N_{j}}
$$

and such that, up to subsequences, it converges weakly to $\zeta$ in $W_{\mathrm{loc}}^{1, p}\left(\left(\mathbb{R}^{n-1} \times I\right) \backslash C_{1, \infty} ; \mathbb{R}^{m}\right)$. Moreover, the function $\zeta$ satisfies the following properties

$$
\left\{\begin{array}{l}
D \zeta \in L^{p}\left(\left(\mathbb{R}^{n-1} \times I\right) \backslash C_{1, \infty} ; \mathbb{R}^{m \times n}\right), \\
\zeta-z \in L^{p}\left(0,1 ; L^{p^{*}}\left(\mathbb{R}^{n-1} ; \mathbb{R}^{m}\right)\right) \\
\zeta \in L^{p}\left(-1,0 ; L^{p^{*}}\left(\mathbb{R}^{n-1} ; \mathbb{R}^{m}\right)\right)
\end{array}\right.
$$

Proof. By (7.1), (7.4) and (7.5) we deduce that, for every fixed $\gamma>0$,

$$
\sup _{j \in \mathbb{N}} \int_{\left(B_{\gamma N_{j}}^{n-1} \times I\right) \backslash C_{1, \gamma N_{j}}}\left|\left(D_{\alpha} \zeta_{\gamma, j} \mid \frac{r_{j}}{\delta_{j}} D_{n} \zeta_{\gamma, j}\right)\right|^{p} d x \leq c .
$$

We define

hence,

$$
\tilde{\zeta}_{j}:=\left\{\begin{array}{lll}
z & \text { in } & \left(\mathbb{R}^{n-1} \backslash B_{\gamma N_{j}}^{n-1}\right)^{+}, \\
\zeta_{\gamma, j} & \text { in } & \left(B_{\gamma N_{j}}^{n-1} \times I\right) \backslash C_{1, \gamma N_{j}} \\
0 & \text { in } & \left(\mathbb{R}^{n-1} \backslash B_{\gamma N_{j}}^{n-1}\right)^{-}
\end{array}\right.
$$

$$
\tilde{\zeta}_{j}\left(\cdot, x_{n}\right)-z \in W^{1, p}\left(\mathbb{R}^{n-1} ; \mathbb{R}^{m}\right) \quad \text { for } \quad \text { a.e. } \quad x_{n} \in(0,1)
$$

and

$$
\tilde{\zeta}_{j}\left(\cdot, x_{n}\right) \in W^{1, p}\left(\mathbb{R}^{n-1} ; \mathbb{R}^{m}\right) \text { for } \text { a.e. } x_{n} \in(-1,0) .
$$

Moreover by (7.7) we get that

$$
\int_{\left(\mathbb{R}^{n-1} \times I\right) \backslash C_{1, \infty}}\left|\left(D_{\alpha} \tilde{\zeta}_{j} \mid \frac{r_{j}}{\delta_{j}} D_{n} \tilde{\zeta}_{j}\right)\right|^{p} d x=\int_{\left(B_{\gamma N_{j}}^{n-1} \times I\right) \backslash C_{1, \gamma N_{j}}}\left|\left(D_{\alpha} \zeta_{\gamma, j} \mid \frac{r_{j}}{\delta_{j}} D_{n} \zeta_{\gamma, j}\right)\right|^{p} d x \leq c .
$$

Since $p<n-1$, according to the Sobolev Inequality (see e.g. [1]), there exists a constant $c=c(n, p)>0$ (independent of $x_{n}$ ) such that

$$
\left(\int_{\mathbb{R}^{n-1}}\left|\tilde{\zeta}_{j}\left(x_{\alpha}, x_{n}\right)-z\right|^{p^{*}} d x_{\alpha}\right)^{p / p^{*}} \leq c \int_{\mathbb{R}^{n-1}}\left|D_{\alpha} \tilde{\zeta}_{j}\left(x_{\alpha}, x_{n}\right)\right|^{p} d x_{\alpha}
$$


for a.e. $x_{n} \in(0,1)$, and

$$
\left(\int_{\mathbb{R}^{n-1}}\left|\tilde{\zeta}_{j}\left(x_{\alpha}, x_{n}\right)\right|^{p^{*}} d x_{\alpha}\right)^{p / p^{*}} \leq c \int_{\mathbb{R}^{n-1}}\left|D_{\alpha} \tilde{\zeta}_{j}\left(x_{\alpha}, x_{n}\right)\right|^{p} d x_{\alpha}
$$

for a.e. $x_{n} \in(-1,0)$. If we integrate (7.9) and (7.10) with respect to $x_{n}$, by (7.8) and Remark 7.2, we get that there exist $\zeta_{1} \in L^{p}\left(0,1 ; L^{p^{*}}\left(\mathbb{R}^{n-1} ; \mathbb{R}^{m}\right)\right)$ and $\zeta_{2} \in L^{p}\left(-1,0 ; L^{p^{*}}\left(\mathbb{R}^{n-1} ; \mathbb{R}^{m}\right)\right)$ such that, up to subsequences,

$$
\left\{\begin{array}{lll}
\tilde{\zeta}_{j}-z \rightarrow \zeta_{1} & \text { in } & L^{p}\left(0,1 ; L^{p^{*}}\left(\mathbb{R}^{n-1} ; \mathbb{R}^{m}\right)\right), \\
\tilde{\zeta}_{j} \rightarrow \zeta_{2} & \text { in } & L^{p}\left(-1,0 ; L^{p^{*}}\left(\mathbb{R}^{n-1} ; \mathbb{R}^{m}\right)\right), \\
D \tilde{\zeta}_{j} \rightarrow D \zeta_{1} & \text { in } & L^{p}\left(\left(\mathbb{R}^{n-1}\right)^{+} ; \mathbb{R}^{m \times n}\right), \\
D \tilde{\zeta}_{j} \rightarrow D \zeta_{2} & \text { in } & L^{p}\left(\left(\mathbb{R}^{n-1}\right)^{-} ; \mathbb{R}^{m \times n}\right) .
\end{array}\right.
$$

In particular, we have that

$$
\left\{\begin{array}{lll}
\tilde{\zeta}_{j} \rightarrow \zeta_{1}+z & \text { in } & W_{\text {loc }}^{1, p}\left(\left(\mathbb{R}^{n-1}\right)^{+} ; \mathbb{R}^{m}\right), \\
\tilde{\zeta}_{j} \rightarrow \zeta_{2} & \text { in } & W_{\text {loc }}^{1, p}\left(\left(\mathbb{R}^{n-1}\right)^{-} ; \mathbb{R}^{m}\right) .
\end{array}\right.
$$

Then, since $\zeta_{1}+z=\zeta_{2}$ on $B_{1}^{n-1}$ in the sense of traces, we can define

$$
\zeta:=\left\{\begin{array}{lll}
\zeta_{1}+z & \text { in } & \left(\mathbb{R}^{n-1}\right)^{+} \\
\zeta_{2} & \text { in } & \left(\mathbb{R}^{n-1}\right)^{-} \cup\left(B_{1}^{n-1} \times\{0\}\right),
\end{array}\right.
$$

and it satisfies (7.6).

Now we are able to describe the interfacial energy density $\varphi^{(\ell)}$ as the following nonlinear capacitary formula.

Proposition 7.4 (Representation formula). We have

$$
\begin{array}{r}
\varphi^{(\ell)}(z)=\inf \left\{\int_{\left(\mathbb{R}^{n-1} \times I\right) \backslash C_{1, \infty}} g\left(D_{\alpha} \zeta \mid \ell D_{n} \zeta\right) d x: \zeta \in W_{\text {loc }}^{1, p}\left(\left(\mathbb{R}^{n-1} \times I\right) \backslash C_{1, \infty} ; \mathbb{R}^{m}\right),\right. \\
D \zeta \in L^{p}\left(\left(\mathbb{R}^{n-1} \times I\right) \backslash C_{1, \infty} ; \mathbb{R}^{m \times n}\right), \zeta-z \in L^{p}\left(0,1 ; L^{p^{*}}\left(\mathbb{R}^{n-1} ; \mathbb{R}^{m}\right)\right) \\
\text { and } \left.\zeta \in L^{p}\left(-1,0 ; L^{p^{*}}\left(\mathbb{R}^{n-1} ; \mathbb{R}^{m}\right)\right)\right\}
\end{array}
$$

for every $z \in \mathbb{R}^{m}$.

Proof. We define

$$
\begin{array}{r}
\psi^{(\ell)}(z):=\inf \left\{\int_{\left(\mathbb{R}^{n-1} \times I\right) \backslash C_{1, \infty}} g\left(D_{\alpha} \zeta \mid \ell D_{n} \zeta\right) d x: \zeta \in W_{\mathrm{loc}}^{1, p}\left(\left(\mathbb{R}^{n-1} \times I\right) \backslash C_{1, \infty} ; \mathbb{R}^{m}\right),\right. \\
D \zeta \in L^{p}\left(\left(\mathbb{R}^{n-1} \times I\right) \backslash C_{1, \infty} ; \mathbb{R}^{m \times n}\right), \zeta-z \in L^{p}\left(0,1 ; L^{p^{*}}\left(\mathbb{R}^{n-1} ; \mathbb{R}^{m}\right)\right) \\
\text { and } \left.\zeta \in L^{p}\left(-1,0 ; L^{p^{*}}\left(\mathbb{R}^{n-1} ; \mathbb{R}^{m}\right)\right)\right\},
\end{array}
$$

we want to prove that $\varphi^{(\ell)}(z)=\psi^{(\ell)}(z)$ for every $z \in \mathbb{R}^{m}$. For every fixed $\eta>0$, by definition of $\varphi_{\gamma, j}^{(\ell)}(z)$ (see (5.2)), there exists $\zeta_{\gamma, j} \in X_{j}^{\gamma}(z)$ such that

$$
\int_{\left(B_{\gamma N_{j}}^{n-1} \times I\right) \backslash C_{1, \gamma N_{j}}} g_{j}\left(D_{\alpha} \zeta_{\gamma, j} \mid \frac{r_{j}}{\delta_{j}} D_{n} \zeta_{\gamma, j}\right) d x \leq \varphi_{\gamma, j}^{(\ell)}(z)+\eta .
$$


By Proposition 5.1(i) we have that (7.5) is fulfilled, then by Propositions 7.3 and 7.1 we get

$$
\begin{aligned}
\lim _{j \rightarrow+\infty} \varphi_{\gamma, j}^{(\ell)}(z)+\eta & \geq \liminf _{j \rightarrow+\infty} \int_{\left(B_{\gamma N_{j}}^{n-1} \times I\right) \backslash C_{1, \gamma N_{j}}} g_{j}\left(D_{\alpha} \tilde{\zeta}_{j} \mid \frac{r_{j}}{\delta_{j}} D_{n} \tilde{\zeta}_{j}\right) d x \\
& \geq \liminf _{j \rightarrow+\infty} \int_{\left(B_{N}^{n-1} \times I\right) \backslash C_{1, N}} g_{j}\left(D_{\alpha} \tilde{\zeta}_{j} \mid \frac{r_{j}}{\delta_{j}} D_{n} \tilde{\zeta}_{j}\right) d x \\
& \geq \int_{\left(B_{N}^{n-1} \times I\right) \backslash C_{1, N}} g\left(D_{\alpha} \zeta \mid \ell D_{n} \zeta\right) d x
\end{aligned}
$$

with $\zeta \in W_{\text {loc }}^{1, p}\left(\left(\mathbb{R}^{n-1} \times I\right) \backslash C_{1, \infty} ; \mathbb{R}^{m}\right)$ satisfying (7.6). Note that for every fixed $\gamma>0$ and $j$ large enough we can always assume that $\gamma N_{j}>N$ for some fixed $N>2$. Hence, passing to the limit as $N \rightarrow+\infty$ and $\gamma \rightarrow 0^{+}$, we obtain

$$
\varphi^{(\ell)}(z)+\eta \geq \int_{\left(\mathbb{R}^{n-1} \times I\right) \backslash C_{1, \infty}} g\left(D_{\alpha} \zeta \mid \ell D_{n} \zeta\right) d x \geq \psi^{(\ell)}(z)
$$

and by the arbitrariness of $\eta$ we get the first inequality.

We now prove the converse inequality. For every fixed $\eta>0$ there exists $\zeta \in W_{\text {loc }}^{1, p}\left(\left(\mathbb{R}^{n-1} \times I\right) \backslash\right.$ $\left.C_{1, \infty} ; \mathbb{R}^{m}\right)$ satisfying $(7.6)$ such that

$$
\int_{\left(\mathbb{R}^{n-1} \times I\right) \backslash C_{1, \infty}} g\left(D_{\alpha} \zeta \mid \ell D_{n} \zeta\right) d x \leq \psi^{(\ell)}(z)+\eta
$$

Let $N>2$, for every fixed $\gamma>0$ and $j$ large enough we have that $\gamma N_{j}>N$. We consider a cut-off function $\theta_{N} \in \mathcal{C}_{c}^{\infty}\left(B_{N}^{n-1} ;[0,1]\right)$ such that $\theta_{N}=1$ in $B_{N / 2}^{n-1},\left|D_{\alpha} \theta_{N}\right| \leq c / N$ and we define

$$
\zeta_{N}:= \begin{cases}\theta_{N}\left(x_{\alpha}\right) \zeta+\left(1-\theta_{N}\left(x_{\alpha}\right)\right) z & \text { in }\left(B_{N}^{n-1}\right)^{+} \\ \theta_{N}\left(x_{\alpha}\right) \zeta & \text { in }\left(B_{N}^{n-1}\right)^{-} \cup\left(B_{1}^{n-1} \times\{0\}\right)\end{cases}
$$

so that $\zeta_{N} \in X_{N}(z)$. By Proposition 7.1, there exists a sequence $\left(\zeta_{j}^{N}\right) \subset X_{N}(z)$ strongly converging to $\zeta_{N}$ in $L^{p}\left(\left(B_{N}^{n-1} \times I\right) \backslash C_{1, N} ; \mathbb{R}^{m}\right)$ such that

$$
\int_{\left(B_{N}^{n-1} \times I\right) \backslash C_{1, N}} g\left(D_{\alpha} \zeta_{N} \mid \ell D_{n} \zeta_{N}\right) d x=\lim _{j \rightarrow+\infty} \int_{\left(B_{N}^{n-1} \times I\right) \backslash C_{1, N}} g_{j}\left(D_{\alpha} \zeta_{j}^{N} \mid \frac{r_{j}}{\delta_{j}} D_{n} \zeta_{j}^{N}\right) d x
$$

Let us define $\zeta_{\gamma, j} \in X_{j}^{\gamma}(z)$ as

$$
\zeta_{\gamma, j}:=\left\{\begin{array}{lll}
z & \text { in } & \left(B_{\gamma N_{j}}^{n-1} \backslash B_{N}^{n-1}\right)^{+} \\
\zeta_{j}^{N} & \text { in } \quad\left(B_{N}^{n-1} \times I\right) \backslash C_{1, N} \\
0 & \text { in } & \left(B_{\gamma N_{j}}^{n-1} \backslash B_{N}^{n-1}\right)^{-} .
\end{array}\right.
$$

Consequently, $\zeta_{\gamma, j}$ is an admissible test function for $(5.2)$ and since $g_{j}(0)=0$ we get that

$$
\begin{aligned}
\varphi_{\gamma, j}^{(\ell)}(z) & \leq \int_{\left(B_{\gamma N_{j}}^{n-1} \times I\right) \backslash C_{1, \gamma N_{j}}} g_{j}\left(D_{\alpha} \zeta_{\gamma, j} \mid \frac{r_{j}}{\delta_{j}} D_{n} \zeta_{\gamma, j}\right) d x \\
& =\int_{\left(B_{N}^{n-1} \times I\right) \backslash C_{1, N}} g_{j}\left(D_{\alpha} \zeta_{N}^{j} \mid \frac{r_{j}}{\delta_{j}} D_{n} \zeta_{N}^{j}\right) d x .
\end{aligned}
$$


Passing to the limit as $j \rightarrow+\infty$, using (7.13) and the $p$-growth condition (7.2) satisfied by $g$, we obtain

$$
\begin{aligned}
\lim _{j \rightarrow+\infty} \varphi_{\gamma, j}^{(\ell)}(z) \leq & \int_{\left(B_{N}^{n-1} \times I\right) \backslash C_{1, N}} g\left(D_{\alpha} \zeta_{N} \mid \ell D_{n} \zeta_{N}\right) d x \\
\leq & \int_{\left(B_{N / 2}^{n-1} \times I\right) \backslash C_{1, N / 2}} g\left(D_{\alpha} \zeta \mid \ell D_{n} \zeta\right) d x+c \int_{\left(B_{N}^{n-1} \backslash B_{N / 2}^{n-1}\right)^{+}}\left|D \zeta_{N}\right|^{p} d x \\
& \quad+c \int_{\left(B_{N}^{n-1} \backslash B_{N / 2}^{n-1}\right)^{-}}\left|D \zeta_{N}\right|^{p} d x .
\end{aligned}
$$

Let us examine the contribution of the gradient in (7.14),

$$
\begin{aligned}
\int_{\left(B_{N}^{n-1} \backslash B_{N / 2}^{n-1}\right)^{+}} & \left|D \zeta_{N}\right|^{p} d x+\int_{\left(B_{N}^{n-1} \backslash B_{N / 2}^{n-1}\right)^{-}}\left|D \zeta_{N}\right|^{p} d x \\
\leq & c \int_{\left(B_{N}^{n-1} \backslash B_{N / 2}^{n-1}\right)^{+}}\left(\left|D_{\alpha} \theta_{N}\right|^{p}|\zeta-z|^{p}+|D \zeta|^{p}\right) d x \\
& +c \int_{\left(B_{N}^{n-1} \backslash B_{N / 2}^{n-1}\right)^{-}}\left(\left|D_{\alpha} \theta_{N}\right|^{p}|\zeta|^{p}+|D \zeta|^{p}\right) d x \\
\leq & \left(\int_{\left(\mathbb{R}^{n-1} \backslash B_{N / 2}^{n-1}\right)^{+}}|D \zeta|^{p} d x+\int_{\left(\mathbb{R}^{n-1} \backslash B_{N / 2}^{n-1}\right)^{-}}|D \zeta|^{p} d x\right) \\
& \quad+\frac{c}{N^{p}}\left(\int_{\left(B_{N}^{n-1} \backslash B_{N / 2}^{n-1}\right)^{+}}|\zeta-z|^{p} d x+\int_{\left(B_{N}^{n-1} \backslash B_{N / 2}^{n-1}\right)^{-}}|\zeta|^{p} d x\right) .
\end{aligned}
$$

Since $p^{*}>p$ we can apply Hölder Inequality with $q=p^{*} / p$ obtaining

$$
\begin{gathered}
\frac{c}{N^{p}}\left(\int_{\left(B_{N}^{n-1} \backslash B_{N / 2}^{n-1}\right)^{+}}|\zeta-z|^{p} d x+\int_{\left(B_{N}^{n-1} \backslash B_{N / 2}^{n-1}\right)^{-}}|\zeta|^{p}\right) \\
\leq c \int_{0}^{1}\left(\int_{B_{N}^{n-1} \backslash B_{N / 2}^{n-1}}|\zeta-z|^{p^{*}} d x_{\alpha}\right)^{p / p^{*}} d x_{n} \\
+c \int_{-1}^{0}\left(\int_{B_{N}^{n-1} \backslash B_{N / 2}^{n-1}}|\zeta|^{p^{*}} d x_{\alpha}\right)^{p / p^{*}} d x_{n} \\
\leq c \int_{0}^{1}\left(\int_{\mathbb{R}^{n-1} \backslash B_{N / 2}^{n-1}}|\zeta-z|^{p^{*}} d x_{\alpha}\right)^{p / p^{*}} d x_{n} \\
+c \int_{-1}^{0}\left(\int_{\mathbb{R}^{n-1} \backslash B_{N / 2}^{n-1}}|\zeta|^{p^{*}} d x_{\alpha}\right)^{p / p^{*}} d x_{n} .
\end{gathered}
$$

Hence by (7.6), (7.15) and (7.16) we have that, for every fixed $\gamma>0$,

$$
\lim _{N \rightarrow+\infty} \int_{\left(B_{N}^{n-1} \backslash B_{N / 2}^{n-1}\right)^{ \pm}}\left|D \zeta_{N}\right|^{p} d x=0
$$

which thanks to (7.12) and (7.14) implies that

$$
\lim _{j \rightarrow+\infty} \varphi_{\gamma, j}^{(\ell)}(z) \leq \psi^{(\ell)}(z)+\eta
$$

Then we get the converse inequality by letting $\gamma \rightarrow 0^{+}$and by the arbitrariness of $\eta$.

7.2. The case $\ell=+\infty$. In this case the study leading to the representation formula for $\varphi^{(\infty)}$ involves a dimensional reduction problem stated on a varying domain. As before, we start proving some $\Gamma$ convergence results (see Propositions 7.5 and 7.7) for suitable sequences of functionals stated on fixed domains. This will allow as to apply some well-known $\Gamma$-convergence and integral representation theorems due to Le Dret-Raoult [23] and Braides-Fonseca-Francfort [13] respectively. 
Let $G_{j}^{ \pm}: L^{p}\left(\left(B_{N}^{n-1}\right)^{ \pm} ; \mathbb{R}^{m}\right) \rightarrow[0,+\infty]$ be defined by

$$
G_{j}^{+}(\zeta):= \begin{cases}\int_{\left(B_{N}^{n-1}\right)^{+}} g_{j}\left(D_{\alpha} \zeta \mid \frac{r_{j}}{\delta_{j}} D_{n} \zeta\right) d x & \text { if }\left\{\begin{array}{l}
\zeta \in W^{1, p}\left(\left(B_{N}^{n-1}\right)^{+} ; \mathbb{R}^{m}\right) \\
\zeta=z \text { on }\left(\partial B_{N}^{n-1}\right)^{+}
\end{array}\right. \\
+\infty & \text { otherwise }\end{cases}
$$

and

$$
G_{j}^{-}(\zeta):= \begin{cases}\int_{\left(B_{N}^{n-1}\right)^{-}} g_{j}\left(D_{\alpha} \zeta \mid \frac{r_{j}}{\delta_{j}} D_{n} \zeta\right) d x & \text { if }\left\{\begin{array}{l}
\zeta \in W^{1, p}\left(\left(B_{N}^{n-1}\right)^{-} ; \mathbb{R}^{m}\right) \\
\zeta=0 \text { on }\left(\partial B_{N}^{n-1}\right)^{-} \\
+\infty
\end{array}\right.\end{cases}
$$

Proposition 7.5. Let

$$
\ell=\lim _{j \rightarrow+\infty} \frac{r_{j}}{\delta_{j}}=+\infty,
$$

then, the sequences of functionals $\left(G_{j}^{ \pm}\right) \Gamma$-converge, with respect to the $L^{p}$-convergence, to

$$
G^{+}(\zeta):= \begin{cases}\int_{B_{N}^{n-1}} \mathcal{Q}_{n-1} \bar{g}\left(D_{\alpha} \zeta\right) d x_{\alpha} & \text { if } \zeta-z \in W_{0}^{1, p}\left(B_{N}^{n-1} ; \mathbb{R}^{m}\right) \\ +\infty & \text { otherwise }\end{cases}
$$

and

$$
G^{-}(\zeta):= \begin{cases}\int_{B_{N}^{n-1}} \mathcal{Q}_{n-1} \bar{g}\left(D_{\alpha} \zeta\right) d x_{\alpha} & \text { if } \zeta \in W_{0}^{1, p}\left(B_{N}^{n-1} ; \mathbb{R}^{m}\right) \\ +\infty & \text { otherwise, }\end{cases}
$$

respectively, where $\bar{g}(\bar{F})=\inf \left\{g\left(\bar{F} \mid F_{n}\right): F_{n} \in \mathbb{R}^{m}\right\}$ for every $\bar{F} \in \mathbb{R}^{m \times(n-1)}$.

Proof. We prove the $\Gamma$-convergence result only for $\left(G_{j}^{+}\right)$, the other one being analogous. According to [13] Theorem 2.5 and Lemma 2.6 there exists a continuous function $\hat{g}: \mathbb{R}^{m \times(n-1)} \rightarrow[0,+\infty)$ such that, up to subsequence, $\left(G_{j}^{+}\right) \Gamma$-converges to

$$
G^{+}(\zeta):= \begin{cases}\int_{B_{N}^{n-1}} \hat{g}\left(D_{\alpha} \zeta\right) d x_{\alpha} & \text { if } \zeta-z \in W_{0}^{1, p}\left(B_{N}^{n-1} ; \mathbb{R}^{m}\right) \\ +\infty & \text { otherwise }\end{cases}
$$

Hence, it remains to show that $\hat{g}=\mathcal{Q}_{n-1} \bar{g}$. By [13] Lemma 2.6, it is enough to consider $W^{1, p}$-functions without boundary condition; hence, it will suffice to deal with affine functions. Let $\zeta\left(x_{\alpha}\right):=\bar{F} \cdot x_{\alpha}$, by [13] Theorem 2.5, there exists a sequence $\left(\zeta_{j}\right) \subset W^{1, p}\left(\left(B_{N}^{n-1}\right)^{+} ; \mathbb{R}^{m}\right)$ (the so-called recovery sequence) converging to $\zeta$ in $L^{p}\left(\left(B_{N}^{n-1}\right)^{+} ; \mathbb{R}^{m}\right)$, such that

$$
\hat{g}(\bar{F}) c_{N}=G^{+}(\zeta)=\lim _{j \rightarrow+\infty} \int_{\left(B_{N}^{n-1}\right)^{+}} g_{j}\left(D_{\alpha} \zeta_{j} \mid \frac{r_{j}}{\delta_{j}} D_{n} \zeta_{j}\right) d x
$$

where $c_{N}=\mathcal{H}^{n-1}\left(B_{N}^{n-1}\right)$. Moreover, by [8] Theorem 1.1, we can assume, without loss of generality, that the sequence $\left(\left|\left(D_{\alpha} \zeta_{j} \mid \frac{r_{j}}{\delta_{j}} D_{n} \zeta_{j}\right)\right|^{p}\right)$ is equi-integrable. By (7.17) and (7.1), we have that

$$
\sup _{j \in \mathbb{N}} \int_{\left(B_{N}^{n-1}\right)^{+}}\left|\left(D_{\alpha} \zeta_{j} \mid \frac{r_{j}}{\delta_{j}} D_{n} \zeta_{j}\right)\right|^{p} d x \leq c ;
$$

hence, for every fixed $M>0$, if we define

$$
A_{j}^{M}:=\left\{x \in\left(B_{N}^{n-1}\right)^{+}:\left|\left(D_{\alpha} \zeta_{j}(x) \mid \frac{r_{j}}{\delta_{j}} D_{n} \zeta_{j}(x)\right)\right| \leq M\right\},
$$


we get that $\mathcal{L}^{n}\left(\left(B_{N}^{n-1}\right)^{+} \backslash A_{j}^{M}\right) \leq c / M^{p}$ for some constant $c>0$ independent of $j$ and $M$. Fix $M>0$, by $(7.17)$, we have

$$
\hat{g}(\bar{F}) c_{N} \geq \limsup _{j \rightarrow+\infty} \int_{A_{j}^{M}} g_{j}\left(D_{\alpha} \zeta_{j} \mid \frac{r_{j}}{\delta_{j}} D_{n} \zeta_{j}\right) d x .
$$

Moreover, for all $x \in A_{j}^{M}$,

$$
\left|g_{j}\left(D_{\alpha} \zeta_{j}(x) \mid \frac{r_{j}}{\delta_{j}} D_{n} \zeta_{j}(x)\right)-g\left(D_{\alpha} \zeta_{j}(x) \mid \frac{r_{j}}{\delta_{j}} D_{n} \zeta_{j}(x)\right)\right| \leq \sup _{|F| \leq M}\left|g_{j}(F)-g(F)\right|,
$$

and then,

$$
\begin{aligned}
& \int_{A_{j}^{M}}\left|g_{j}\left(D_{\alpha} \zeta_{j} \mid \frac{r_{j}}{\delta_{j}} D_{n} \zeta_{j}\right)-g\left(D_{\alpha} \zeta_{j} \mid \frac{r_{j}}{\delta_{j}} D_{n} \zeta_{j}\right)\right| d x \\
\leq & c_{N} \sup _{|F| \leq M}\left|g_{j}(F)-g(F)\right| .
\end{aligned}
$$

Hence, by the local uniform convergence of $g_{j}$ to $g$, we have that

$$
\lim _{j \rightarrow+\infty} \int_{A_{j}^{M}}\left(g_{j}\left(D_{\alpha} \zeta_{j} \mid \frac{r_{j}}{\delta_{j}} D_{n} \zeta_{j}\right)-g\left(D_{\alpha} \zeta_{j} \mid \frac{r_{j}}{\delta_{j}} D_{n} \zeta_{j}\right)\right) d x=0 .
$$

By (7.18), we get

$$
\hat{g}(\bar{F}) c_{N} \geq \limsup _{j \rightarrow+\infty} \int_{A_{j}^{M}} g\left(D_{\alpha} \zeta_{j} \mid \frac{r_{j}}{\delta_{j}} D_{n} \zeta_{j}\right) d x .
$$

Note that, since $\mathcal{L}^{n}\left(\left(B_{N}^{n-1}\right)^{+} \backslash A_{j}^{M}\right) \rightarrow 0$ as $M \rightarrow+\infty$, by the $p$-growth condition (7.2) and the equiintegrability assumption, we find

$$
\limsup _{j \rightarrow+\infty} \int_{\left(B_{N}^{n-1}\right)^{+} \backslash A_{j}^{M}} g\left(D_{\alpha} \zeta_{j} \mid \frac{r_{j}}{\delta_{j}} D_{n} \zeta_{j}\right) d x=o(1), \quad \text { as } \quad M \rightarrow+\infty .
$$

Consequently, (7.19) and (7.20) imply that

$$
\hat{g}(\bar{F}) c_{N} \geq \limsup _{j \rightarrow+\infty} \int_{\left(B_{N}^{n-1}\right)^{+}} g\left(D_{\alpha} \zeta_{j} \mid \frac{r_{j}}{\delta_{j}} D_{n} \zeta_{j}\right) d x .
$$

Finally, from [23] Theorem 2, we know that

$$
\liminf _{j \rightarrow+\infty} \int_{\left(B_{N}^{n-1}\right)^{+}} g\left(D_{\alpha} \zeta_{j} \mid \frac{r_{j}}{\delta_{j}} D_{n} \zeta_{j}\right) d x \geq \mathcal{Q}_{n-1} \bar{g}(\bar{F}) c_{N}
$$

hence, by (7.21) we obtain that $\hat{g}(\bar{F}) \geq \mathcal{Q}_{n-1} \bar{g}(\bar{F})$.

We now prove the converse inequality. By [23] Theorem 2, there exists a sequence $\left(\zeta_{j}\right)$ belonging to $W^{1, p}\left(\left(B_{N}^{n-1}\right)^{+} ; \mathbb{R}^{m}\right)$ and converging to $\zeta$ in $L^{p}\left(\left(B_{N}^{n-1}\right)^{+} ; \mathbb{R}^{m}\right)$ such that

$$
\mathcal{Q}_{n-1} \bar{g}(\bar{F}) c_{N}=\lim _{j \rightarrow+\infty} \int_{\left(B_{N}^{n-1}\right)^{+}} g\left(D_{\alpha} \zeta_{j} \mid \frac{r_{j}}{\delta_{j}} D_{n} \zeta_{j}\right) d x .
$$

Without loss of generality, we can still assume that the sequence $\left(\left|\left(D_{\alpha} \zeta_{j} \mid \frac{r_{j}}{\delta_{j}} D_{n} \zeta_{j}\right)\right|^{p}\right)$ is equi-integrable. Thus arguing as above, from (7.22) we deduce

$$
\mathcal{Q}_{n-1} \bar{g}(\bar{F}) c_{N} \geq \limsup _{j \rightarrow+\infty} \int_{\left(B_{N}^{n-1}\right)^{+}} g_{j}\left(D_{\alpha} \zeta_{j} \mid \frac{r_{j}}{\delta_{j}} D_{n} \zeta_{j}\right) d x .
$$

Now, by [13] Theorem 2.5, we have that

$$
\liminf _{j \rightarrow+\infty} \int_{\left(B_{N}^{n-1}\right)^{+}} g_{j}\left(D_{\alpha} \zeta_{j} \mid \frac{r_{j}}{\delta_{j}} D_{n} \zeta_{j}\right) d x \geq \hat{g}(\bar{F}) c_{N} ;
$$

hence, $\mathcal{Q}_{n-1} \bar{g}(\bar{F}) \geq \hat{g}(\bar{F})$, which concludes the proof. 
Remark 7.6. By [23] Theorem 2 , for every $\zeta \in W^{1, p}\left(B_{N}^{n-1} ; \mathbb{R}^{m}\right)$ the recovery sequence is given by $\zeta_{j}\left(x_{\alpha}, x_{n}\right):=\zeta\left(x_{\alpha}\right)+\left(\delta_{j} / r_{j}\right) x_{n} b_{j}\left(x_{\alpha}\right)$ for a suitable sequence of functions $\left(b_{j}\right) \subset \mathcal{C}_{c}^{\infty}\left(B_{N}^{n-1} ; \mathbb{R}^{m}\right)$. Note that by definition $\left(\zeta_{j}\right)$ keeps the boundary conditions of $\zeta$. Reasoning as in the proof of Proposition 7.5 we can observed that $\left(\zeta_{j}\right)$ is also a recovery sequence for $\left(G_{j}^{+}\right)$(see e.g. (7.23)). The same remark holds for $\left(G_{j}^{-}\right)$.

Proposition 7.7. Let

$$
\ell=\lim _{j \rightarrow+\infty} \frac{r_{j}}{\delta_{j}}=+\infty
$$

then the sequence of functionals $G_{j}^{(\infty)}: L^{p}\left(\left(B_{N}^{n-1} \times I\right) \backslash C_{1, N} ; \mathbb{R}^{m}\right) \rightarrow[0,+\infty]$ defined by

$$
G_{j}^{(\infty)}(\zeta):= \begin{cases}\int_{\left(B_{N}^{n-1} \times I\right) \backslash C_{1, N}} g_{j}\left(D_{\alpha} \zeta \mid \frac{r_{j}}{\delta_{j}} D_{n} \zeta\right) d x & \text { if } \zeta \in X_{N}(z) \\ +\infty & \text { otherwise }\end{cases}
$$

$\Gamma$-converges, with respect to the $L^{p}$-convergence, to

$$
G^{(\infty)}(\zeta):= \begin{cases}\int_{\left(B_{N}^{n-1} \times I\right) \backslash C_{1, N}} \mathcal{Q}_{n-1} \bar{g}\left(D_{\alpha} \zeta\right) d x & \text { if } \zeta \in X_{N}(z) \text { and } D_{n} \zeta=0 \\ +\infty & \text { otherwise. }\end{cases}
$$

Proof. The lim inf inequality is a straightforward consequence of Proposition 7.5.

Dealing with the limsup inequality, let us consider $\zeta \in X_{N}(z)$ with $D_{n} \zeta=0$. We denote by $\zeta^{ \pm} \in$ $W^{1, p}\left(B_{N}^{n-1}(0) ; \mathbb{R}^{m}\right)$ the restriction of $\zeta$ to $\left(B_{N}^{n-1}\right)^{+}$and $\left(B_{N}^{n-1}\right)^{-}$, respectively. By Proposition 7.5 and Remark 7.6, there exist two sequences $\left(\zeta_{j}^{ \pm}\right) \subset W^{1, p}\left(\left(B_{N}^{n-1}\right)^{ \pm} ; \mathbb{R}^{m}\right)$ such that

$$
\begin{aligned}
& \zeta_{j}^{+} \rightarrow \zeta^{+} \text {in } L^{p}\left(\left(B_{N}^{n-1}\right)^{+} ; \mathbb{R}^{m}\right), \quad \zeta_{j}^{+}=z \text { on }\left(\partial B_{N}^{n-1}\right)^{+} \\
& \zeta_{j}^{-} \rightarrow \zeta^{-} \text {in } L^{p}\left(\left(B_{N}^{n-1}\right)^{-} ; \mathbb{R}^{m}\right), \quad \zeta_{j}^{-}=0 \text { on }\left(\partial B_{N}^{n-1}\right)^{-}
\end{aligned}
$$

and

$$
\begin{aligned}
& \lim _{j \rightarrow+\infty} \int_{\left(B_{N}^{n-1}\right)^{+}} g_{j}\left(D_{\alpha} \zeta_{j}^{+} \mid \frac{r_{j}}{\delta_{j}} D_{n} \zeta_{j}^{+}\right) d x=\int_{B_{N}^{n-1}} \mathcal{Q}_{n-1} \bar{g}\left(D_{\alpha} \zeta^{+}\right) d x_{\alpha} \\
& \lim _{j \rightarrow+\infty} \int_{\left(B_{N}^{n-1}\right)^{-}} g_{j}\left(D_{\alpha} \zeta_{j}^{-} \mid \frac{r_{j}}{\delta_{j}} D_{n} \zeta_{j}^{-}\right) d x=\int_{B_{N}^{n-1}} \mathcal{Q}_{n-1} \bar{g}\left(D_{\alpha} \zeta^{-}\right) d x_{\alpha} .
\end{aligned}
$$

Moreover, since $\zeta \in W^{1, p}\left(\left(B_{N}^{n-1} \times I\right) \backslash C_{1, N} ; \mathbb{R}^{m}\right)$, by Remark 7.6, $\left(\zeta_{j}^{+}\right)$and $\left(\zeta_{j}^{-}\right)$have the same trace on $B_{1}^{n-1} \times\{0\}$; hence, $\zeta_{j}^{+}=\zeta_{j}^{-}=\zeta$ on $B_{1}^{n-1} \times\{0\}$. Then we can define

$$
\bar{\zeta}_{j}:=\left\{\begin{array}{lll}
\zeta_{j}^{+} & \text {in } & \left(B_{N}^{n-1}\right)^{+} \\
\zeta & \text { on } & B_{1}^{n-1} \times\{0\} \\
\zeta_{j}^{-} & \text {in } & \left(B_{N}^{n-1}\right)^{-}
\end{array}\right.
$$

with $\bar{\zeta}_{j} \in W^{1, p}\left(\left(B_{N}^{n-1} \times I\right) \backslash C_{1, N} ; \mathbb{R}^{m}\right)$. In particular, by (7.24) we have that $\bar{\zeta}_{j} \in X_{N}(z)$ and $\bar{\zeta}_{j} \rightarrow \zeta$ in $L^{p}\left(\left(B_{N}^{n-1} \times I\right) \backslash C_{1, N} ; \mathbb{R}^{m}\right)$. Finally, by (7.25), we have

$$
\begin{aligned}
\lim _{j \rightarrow+\infty} G_{j}^{(\infty)}\left(\bar{\zeta}_{j}\right) & =\lim _{j \rightarrow+\infty} \int_{\left(B_{N}^{n-1} \times I\right) \backslash C_{1, N}} g_{j}\left(D_{\alpha} \bar{\zeta}_{j} \mid \frac{r_{j}}{\delta_{j}} D_{n} \bar{\zeta}_{j}\right) d x \\
& =\int_{B_{N}^{n-1}} \mathcal{Q}_{n-1} \bar{g}\left(D_{\alpha} \zeta^{+}\right) d x_{\alpha}+\int_{B_{N}^{n-1}} \mathcal{Q}_{n-1} \bar{g}\left(D_{\alpha} \zeta^{-}\right) d x_{\alpha} \\
& =\int_{\left(B_{N}^{n-1} \times I\right) \backslash C_{1, N}} \mathcal{Q}_{n-1} \bar{g}\left(D_{\alpha} \zeta\right) d x
\end{aligned}
$$


which completes the proof of the limsup inequality.

Proposition 7.8 (Limit space). Let

$$
\ell=\lim _{j \rightarrow+\infty} \frac{r_{j}}{\delta_{j}}=+\infty, \quad 0<R^{(\infty)}=\lim _{j \rightarrow+\infty} \frac{r_{j}^{n-1-p}}{\varepsilon_{j}^{n-1}}<+\infty
$$

and let $\zeta_{\gamma, j} \in X_{j}^{\gamma}(z)$ such that, for every fixed $\gamma>0$,

$$
\sup _{j \in \mathbb{N}} \int_{\left(B_{\gamma N_{j}}^{n-1} \times I\right) \backslash C_{1, \gamma N_{j}}} g_{j}\left(D_{\alpha} \zeta_{\gamma, j} \mid \frac{r_{j}}{\delta_{j}} D_{n} \zeta_{\gamma, j}\right) d x \leq c .
$$

Then, there exists a sequence $\tilde{\zeta}_{j} \in W_{\mathrm{loc}}^{1, p}\left(\left(\mathbb{R}^{n-1} \times I\right) \backslash C_{1, \infty} ; \mathbb{R}^{m}\right)$ such that

$$
\tilde{\zeta}_{j}=\zeta_{\gamma, j} \quad \text { in } \quad\left(B_{\gamma N_{j}}^{n-1} \times I\right) \backslash C_{1, \gamma N_{j}}
$$

and such that, up to subsequences, it converges weakly to $\zeta^{+}$in $W_{\text {loc }}^{1, p}\left(\left(\mathbb{R}^{n-1}\right)^{+} ; \mathbb{R}^{m}\right)$ and to $\zeta^{-}$in $W_{\text {loc }}^{1, p}\left(\left(\mathbb{R}^{n-1}\right)^{-} ; \mathbb{R}^{m}\right)$. Moreover, the functions $\zeta^{ \pm}$satisfy the following properties

$$
\left\{\begin{array}{l}
\zeta^{ \pm} \in W_{\text {loc }}^{1, p}\left(\mathbb{R}^{n-1} ; \mathbb{R}^{m}\right), \\
\zeta^{+}=\zeta^{-} \quad \text { in } B_{1}^{n-1} \\
D_{\alpha} \zeta^{ \pm} \in L^{p}\left(\mathbb{R}^{n-1} ; \mathbb{R}^{m \times(n-1)}\right), \\
\left(\zeta^{+}-z\right) \text { and } \zeta^{-} \in L^{p^{*}}\left(\mathbb{R}^{n-1} ; \mathbb{R}^{m}\right) .
\end{array}\right.
$$

Proof. We can reason as in Proposition 7.3 using the fact that, by (7.26),

$$
\int_{\left(\mathbb{R}^{n-1}\right)^{ \pm}}\left|D_{n} \tilde{\zeta}_{j}\right|^{p} d x \leq c\left(\frac{\delta_{j}}{r_{j}}\right)^{p}
$$

hence, in the limit we have that $D_{n} \zeta=0$ a.e. in $\left(\mathbb{R}^{n-1}\right)^{ \pm}$.

Proposition 7.9 (Representation formula). We have

$$
\begin{array}{r}
\varphi^{(\infty)}(z)=\inf \left\{\int_{\mathbb{R}^{n-1}}\left(\mathcal{Q}_{n-1} \bar{g}\left(D_{\alpha} \zeta^{+}\right)+\mathcal{Q}_{n-1} \bar{g}\left(D_{\alpha} \zeta^{-}\right)\right) d x_{\alpha}: \zeta^{ \pm} \in W_{\mathrm{loc}}^{1, p}\left(\mathbb{R}^{n-1} ; \mathbb{R}^{m}\right)\right. \\
\zeta^{+}=\zeta^{-} \text {in } B_{1}^{n-1}, \quad D_{\alpha} \zeta^{ \pm} \in L^{p}\left(\mathbb{R}^{n-1} ; \mathbb{R}^{m \times(n-1)}\right) \\
\left.\left(\zeta^{+}-z\right) \text { and } \zeta^{-} \in L^{p^{*}}\left(\mathbb{R}^{n-1} ; \mathbb{R}^{m}\right)\right\}
\end{array}
$$

for every $z \in \mathbb{R}^{m}$.

Proof. Reasoning as in the proof of Proposition 7.4, by Propositions 7.7 and 7.8 we get the representation formula for $\varphi^{(\infty)}$.

7.3. The case $\ell=0$. We first recall the following $\Gamma$-convergence result.

Proposition 7.10. The sequence of functionals $G_{j}^{(0)}: L^{p}\left(\left(B_{N}^{n-1} \times(-N, N)\right) \backslash C_{1, N} ; \mathbb{R}^{m}\right) \rightarrow[0,+\infty]$, defined by

$$
G_{j}^{(0)}(\zeta):= \begin{cases}\int_{\left(B_{N}^{n-1} \times(-N, N)\right) \backslash C_{1, N}} g_{j}(D \zeta) d x & \text { if } \zeta \in W^{1, p}\left(\left(B_{N}^{n-1} \times(-N, N)\right) \backslash C_{1, N} ; \mathbb{R}^{m}\right), \\ +\infty & \text { otherwise, }\end{cases}
$$


$\Gamma$-converges, with respect to the $L^{p}$-convergence, to

$$
G^{(0)}(\zeta):= \begin{cases}\int_{\left(B_{N}^{n-1} \times(-N, N)\right) \backslash C_{1, N}} g(D \zeta) d x & \text { if } \zeta \in W^{1, p}\left(\left(B_{N}^{n-1} \times(-N, N)\right) \backslash C_{1, N} ; \mathbb{R}^{m}\right), \\ +\infty & \text { otherwise. }\end{cases}
$$

Proof. The result is an immediate consequence of the pointwise convergence of the sequence of quasiconvex functions $g_{j}$ towards $g$ together with Proposition 12.8 in [10].

Proposition 7.11 (Limit space). Let

$$
\ell=\lim _{j \rightarrow+\infty} \frac{r_{j}}{\delta_{j}}=0, \quad 0<R^{(0)}=\lim _{j \rightarrow+\infty} \frac{r_{j}^{n-p}}{\varepsilon_{j}^{n-1} \delta_{j}}<+\infty
$$

and let $\zeta_{\gamma, j} \in Y_{j}^{\gamma}(z)$ such that, for every fixed $\gamma>0$,

$$
\sup _{j \in \mathbb{N}} \int_{\left(B_{\gamma N_{j}}^{n-1} \times I_{j}\right) \backslash C_{1, \gamma N_{j}}} g_{j}\left(D \zeta_{\gamma, j}\right) d x \leq c .
$$

Then, there exists a sequence $\tilde{\zeta}_{j} \in W_{\mathrm{loc}}^{1, p}\left(\mathbb{R}^{n} \backslash C_{1, \infty} ; \mathbb{R}^{m}\right)$ such that

$$
\tilde{\zeta}_{j}=\zeta_{\gamma, j} \quad \text { in } \quad\left(B_{\gamma N_{j}}^{n-1} \times I_{j}\right) \backslash C_{1, \gamma N_{j}}
$$

and such that, up to subsequences, it converges weakly to $\zeta$ in $W_{\text {loc }}^{1, p}\left(\mathbb{R}^{n} \backslash C_{1, \infty} ; \mathbb{R}^{m}\right)$. Moreover, the function $\zeta$ satisfies the following properties

$$
\left\{\begin{array}{l}
D \zeta \in L^{p}\left(\mathbb{R}^{n} \backslash C_{1, \infty} ; \mathbb{R}^{m \times n}\right) \\
\zeta-z \in L^{p}\left(0,+\infty ; L^{p^{*}}\left(\mathbb{R}^{n-1} ; \mathbb{R}^{m}\right)\right) \\
\zeta \in L^{p}\left(-\infty, 0 ; L^{p^{*}}\left(\mathbb{R}^{n-1} ; \mathbb{R}^{m}\right)\right)
\end{array}\right.
$$

Proof. By (7.28), (7.1) and (7.27), we deduce that, for every fixed $\gamma>0$,

$$
\sup _{j \in \mathbb{N}} \int_{\left(B_{\gamma N_{j}}^{n-1} \times I_{j}\right) \backslash C_{1, \gamma N_{j}}}\left|D \zeta_{\gamma, j}\right|^{p} d x \leq c .
$$

Let us first extend $\zeta_{\gamma, j}$ by reflection

$$
\bar{\zeta}_{\gamma, j}(x)= \begin{cases}\zeta_{\gamma, j}\left(x_{\alpha}, 2 \frac{\delta_{j}}{r_{j}}-x_{n}\right) & \text { if } \quad x_{\alpha} \in B_{\gamma N_{j}}^{n-1} \text { and } x_{n} \in\left(\delta_{j} / r_{j}, 2 \delta_{j} / r_{j}\right) \\ \zeta_{\gamma, j}(x) & \text { if } \quad x \in\left(B_{\gamma N_{j}}^{n-1} \times I_{j}\right) \backslash C_{1, \gamma N_{j}}, \\ \zeta_{\gamma, j}\left(x_{\alpha},-2 \frac{\delta_{j}}{r_{j}}-x_{n}\right) & \text { if } \quad x_{\alpha} \in B_{\gamma N_{j}}^{n-1} \text { and } x_{n} \in\left(-2 \delta_{j} / r_{j},-\delta_{j} / r_{j}\right)\end{cases}
$$

and then, we extend it by $\left(2 \delta_{j} / r_{j}\right)$-periodicity in the $x_{n}$ direction. The resulting sequence, still denoted by $\bar{\zeta}_{\gamma, j}$, is defined in $\left(B_{\gamma N_{j}}^{n-1} \times \mathbb{R}\right) \backslash C_{1, \gamma N_{j}}$. Hence, we define on $\mathbb{R}^{n} \backslash C_{1, \infty}$,

$$
\bar{\zeta}_{j}(x):=\left\{\begin{array}{lll}
z & \text { in } & \left(\mathbb{R}^{n-1} \backslash B_{\gamma N_{j}}^{n-1}\right) \times(0,+\infty), \\
\bar{\zeta}_{\gamma, j}(x) & \text { in } & \left(B_{\gamma N_{j}}^{n-1} \times \mathbb{R}\right) \backslash C_{1, \gamma N_{j}}, \\
0 & \text { in } & \left(\mathbb{R}^{n-1} \backslash B_{\gamma N_{j}}^{n-1}\right) \times(-\infty, 0) .
\end{array}\right.
$$


Let us now introduce the cut-off functions $\phi_{j} \in \mathcal{C}_{c}^{\infty}\left(\left(-2 \delta_{j} / r_{j}, 2 \delta_{j} / r_{j}\right) ;[0,1]\right)$ such that $\phi_{j}\left(x_{n}\right)=1$ if $\left|x_{n}\right| \leq \delta_{j} / r_{j}, \phi_{j}\left(x_{n}\right)=0$ if $\left|x_{n}\right| \geq 2 \delta_{j} / r_{j}$ and $\left|D_{n} \phi_{j}\right| \leq c\left(r_{j} / \delta_{j}\right)$. Then, we introduce our last sequence,

$$
\tilde{\zeta}_{j}\left(x_{\alpha}, x_{n}\right):=\left\{\begin{array}{lll}
\phi_{j}\left(x_{n}\right) \bar{\zeta}_{j}\left(x_{\alpha}, x_{n}\right)+\left(1-\phi_{j}\left(x_{n}\right)\right) z & \text { if } & \left(x_{\alpha}, x_{n}\right) \in \mathbb{R}^{n-1} \times(0,+\infty), \\
\phi_{j}\left(x_{n}\right) \bar{\zeta}_{j}\left(x_{\alpha}, x_{n}\right) & \text { if } & \left(x_{\alpha}, x_{n}\right) \in \mathbb{R}^{n-1} \times(-\infty, 0) .
\end{array}\right.
$$

Note that

$$
\tilde{\zeta}_{j}=\zeta_{\gamma, j} \quad \text { in } \quad\left(B_{\gamma N_{j}}^{n-1} \times I_{j}\right) \backslash C_{1, \gamma N_{j}} .
$$

Moreover, by (7.30)-(7.33) we have that

$$
\sup _{j \in \mathbb{N}} \int_{\mathbb{R}^{n} \backslash C_{1, \infty}}\left|D_{\alpha} \tilde{\zeta}_{j}\right|^{p} d x \leq c,
$$

while, for every $(a, b) \subset \mathbb{R}$, with $a<b$, we have

$$
\int_{\left(\mathbb{R}^{n-1} \times(a, b)\right) \backslash C_{1, \infty}}\left|D_{n} \tilde{\zeta}_{j}\right|^{p} d x \leq c,
$$

for $j$ large enough and $c$ independent of $(a, b)$. Reasoning as in Proposition 7.3, with $(0,+\infty)$ and $(-\infty, 0)$ in place of $(0,1)$ and $(-1,0)$, respectively, we can conclude that there exist $\zeta_{1} \in L^{p}\left(0,+\infty ; L^{p^{*}}\left(\mathbb{R}^{n-1} ; \mathbb{R}^{m}\right)\right)$ and $\zeta_{2} \in L^{p}\left(-\infty, 0 ; L^{p^{*}}\left(\mathbb{R}^{n-1} ; \mathbb{R}^{m}\right)\right)$ such that, up to subsequences,

$$
\tilde{\zeta}_{j}-z \rightarrow \zeta_{1} \quad \text { in } \quad L^{p}\left(0,+\infty ; L^{p^{*}}\left(\mathbb{R}^{n-1} ; \mathbb{R}^{m}\right)\right)
$$

and

$$
\tilde{\zeta}_{j} \rightarrow \zeta_{2} \quad \text { in } \quad L^{p}\left(-\infty, 0 ; L^{p^{*}}\left(\mathbb{R}^{n-1} ; \mathbb{R}^{m}\right)\right)
$$

Moreover, by (7.34) and (7.35), we have that, up to subsequences, $\tilde{\zeta}_{j}$ converges weakly to $\zeta$ in $W_{\text {loc }}^{1, p}\left(\mathbb{R}^{n} \backslash\right.$ $\left.C_{1, \infty} ; \mathbb{R}^{m}\right)$ where

$$
\zeta=\left\{\begin{array}{lll}
\zeta_{1}+z & \text { in } & \mathbb{R}^{n-1} \times(0,+\infty) \\
\zeta_{2} & \text { in } & \left(\mathbb{R}^{n-1} \times(-\infty, 0)\right) \cup\left(B_{1}^{n-1} \times\{0\}\right)
\end{array}\right.
$$

In particular, for any compact set $K \subset \mathbb{R}^{n} \backslash C_{1, \infty}$, we have that

$$
\int_{K}|D \zeta|^{p} d x \leq \liminf _{j \rightarrow+\infty} \int_{K}\left|D \tilde{\zeta}_{j}\right|^{p} d x \leq c
$$

for some constant $c$ independent of $K$; hence, we get that $D \zeta \in L^{p}\left(\mathbb{R}^{n} \backslash C_{1, \infty} ; \mathbb{R}^{m \times n}\right)$ which concludes the description of the limit function $\zeta$.

Proposition 7.12 (Representation formula). We have

$$
\begin{array}{r}
\varphi^{(0)}(z)=\inf \left\{\int_{\mathbb{R}^{n} \backslash C_{1, \infty}} g(D \zeta) d x: \zeta \in W_{\mathrm{loc}}^{1, p}\left(\mathbb{R}^{n} \backslash C_{1, \infty} ; \mathbb{R}^{m}\right), D \zeta \in L^{p}\left(\mathbb{R}^{n} \backslash C_{1, \infty} ; \mathbb{R}^{m \times n}\right),\right. \\
\left.\zeta-z \in L^{p}\left(0,+\infty ; L^{p^{*}}\left(\mathbb{R}^{n-1} ; \mathbb{R}^{m}\right)\right) \text { and } \zeta \in L^{p}\left(-\infty, 0 ; L^{p^{*}}\left(\mathbb{R}^{n-1} ; \mathbb{R}^{m}\right)\right)\right\}
\end{array}
$$

for every $z \in \mathbb{R}^{m}$.

Proof. We define

$$
\begin{array}{r}
\psi^{(0)}(z):=\inf \left\{\int_{\mathbb{R}^{n} \backslash C_{1, \infty}} g(D \zeta) d x: \zeta \in W_{\mathrm{loc}}^{1, p}\left(\mathbb{R}^{n} \backslash C_{1, \infty} ; \mathbb{R}^{m}\right), D \zeta \in L^{p}\left(\mathbb{R}^{n} \backslash C_{1, \infty} ; \mathbb{R}^{m \times n}\right),\right. \\
\left.\zeta-z \in L^{p}\left(0,+\infty ; L^{p^{*}}\left(\mathbb{R}^{n-1} ; \mathbb{R}^{m}\right)\right) \text { and } \zeta \in L^{p}\left(-\infty, 0 ; L^{p^{*}}\left(\mathbb{R}^{n-1} ; \mathbb{R}^{m}\right)\right)\right\}
\end{array}
$$

and let us prove that $\varphi^{(0)}(z)=\psi^{(0)}(z)$ for every $z \in \mathbb{R}^{m}$. 
By definition of $\varphi_{\gamma, j}^{(0)}$ (see (5.13)), for every fixed $\eta>0$, there exists $\zeta_{\gamma, j} \in Y_{j}^{\gamma}(z)$ such that

$$
\int_{\left(B_{\gamma N_{j}}^{n-1} \times I_{j}\right) \backslash C_{1, \gamma N_{j}}} g_{j}\left(D \zeta_{\gamma, j}\right) d x \leq \varphi_{\gamma, j}^{(0)}(z)+\eta
$$

hence, by Proposition 5.2 (i), (7.28) is satisfied. Then by Propositions 7.10 and 7.11 we get that

$$
\begin{aligned}
\lim _{j \rightarrow+\infty} \varphi_{\gamma, j}^{(0)}(z)+\eta & \geq \liminf _{j \rightarrow+\infty} \int_{\left(B_{\gamma N_{j}}^{n-1} \times I_{j}\right) \backslash C_{1, \gamma N_{j}}} g_{j}\left(D \tilde{\zeta}_{j}\right) d x \\
& \geq \liminf _{j \rightarrow+\infty} \int_{\left(B_{N}^{n-1} \times(-N, N)\right) \backslash C_{1, N}} g_{j}\left(D \tilde{\zeta}_{j}\right) d x \\
& \geq \int_{\left(B_{N}^{n-1} \times(-N, N)\right) \backslash C_{1, N}} g(D \zeta) d x
\end{aligned}
$$

for some fixed $N>1$, where $\zeta$ satisfies (7.29). Thus, passing to the limit in (7.37) as $N \rightarrow+\infty, \gamma \rightarrow 0^{+}$ and $\eta \rightarrow 0^{+}$, it follows that

$$
\varphi^{(0)}(z) \geq \int_{\mathbb{R}^{n} \backslash C_{1, \infty}} g(D \zeta) d x \geq \psi^{(0)}(z)
$$

Let us prove the converse inequality. For any fixed $\eta>0$, let $\zeta \in W_{\text {loc }}^{1, p}\left(\mathbb{R}^{n} \backslash C_{1, \infty} ; \mathbb{R}^{m}\right)$ be as in $(7.29)$ and satisfying

$$
\int_{\mathbb{R}^{n} \backslash C_{1, \infty}} g(D \zeta) d x \leq \psi^{(0)}(z)+\eta
$$

For every $j \in \mathbb{N}$ and $\gamma>0$, we consider a cut-off function $\theta_{\gamma, j} \in \mathcal{C}_{c}^{\infty}\left(B_{\gamma N_{j}}^{n-1} ;[0,1]\right)$ such that $\theta_{\gamma, j}=1$ in $B_{\left(\gamma N_{j}\right) / 2}^{n-1},\left|D_{\alpha} \theta_{\gamma, j}\right| \leq c / \gamma N_{j}$ and we define $\zeta_{\gamma, j} \in Y_{j}^{\gamma}(z)$ by

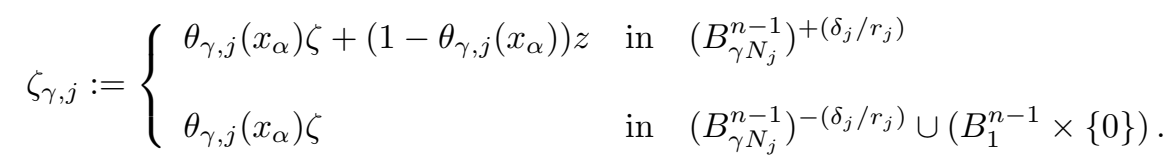

Consequently, $\zeta_{\gamma, j}$ is an admissible test function for (5.13) and we get that

$$
\varphi_{\gamma, j}^{(0)}(z) \leq \int_{\left(B_{\gamma N_{j}}^{n-1} \times I_{j}\right) \backslash C_{1, \gamma N_{j}}} g_{j}\left(D \zeta_{\gamma, j}\right) d x
$$

The same kind of computations as those already employed in the proof of Lemma 7.4 now with $g_{j}$ in place of $g$ and with other obvious replacements (see (7.14)-(7.16)) gives

$$
\lim _{j \rightarrow+\infty} \varphi_{\gamma, j}^{(0)}(z) \leq \limsup _{j \rightarrow+\infty} \int_{\left(B_{\gamma N_{j}}^{n-1} \times I_{j}\right) \backslash C_{1, \gamma N_{j}}} g_{j}(D \zeta) d x+o(1), \quad \text { as } \quad \gamma \rightarrow 0^{+} .
$$

On the other hand, Fatou's Lemma and (7.1) imply

$$
\limsup _{j \rightarrow+\infty} \int_{\left(B_{\gamma N_{j}}^{n-1} \times I_{j}\right) \backslash C_{1, \gamma N_{j}}} g_{j}(D \zeta) d x \leq \int_{\mathbb{R}^{n} \backslash C_{1, \infty}} g(D \zeta) d x+o(1), \quad \text { as } \quad \gamma \rightarrow 0^{+} .
$$

Hence by (7.38), passing to the limit as $\gamma \rightarrow 0^{+}$, we get that

$$
\varphi^{(0)}(z) \leq \psi^{(0)}(z)+\eta
$$

and by the arbitrariness of $\eta$, the thesis. 
Remark 7.13. As already recalled, in [2] it is proved that if $\delta_{j}=1$ or $\delta_{j}=\varepsilon_{j}$ then the critical size $r_{j}$ of the contact zones is of order $\varepsilon_{j}^{(n-1) /(n-p)}$ or $\varepsilon_{j}^{n /(n-p)}$, respectively; moreover, the interfacial energy density is described by the following formula

$$
\begin{aligned}
\varphi(z)=\inf \left\{\int_{\mathbb{R}^{n} \backslash C_{1, \infty}} g(D \zeta) d x: \quad \zeta\right. & \in W_{\text {loc }}^{1, p}\left(\mathbb{R}^{n} \backslash C_{1, \infty} ; \mathbb{R}^{m}\right) \\
& \left.\zeta-z \in W^{1, p}\left(\mathbb{R}_{+}^{n} ; \mathbb{R}^{m}\right), \quad \zeta \in W^{1, p}\left(\mathbb{R}_{-}^{n} ; \mathbb{R}^{m}\right)\right\}
\end{aligned}
$$

where $\mathbb{R}_{+}^{n}=\mathbb{R}^{n-1} \times(0,+\infty), \mathbb{R}_{-}^{n}=\mathbb{R}^{n-1} \times(-\infty, 0)$ (see [2] Section 7 , the case $p=q$, with $\rho_{\varepsilon_{j}}=r_{j}$, $W_{p}=U_{p}=W, \widehat{W}_{p}=\widehat{U}_{p}=g$ and $\left.\mathbb{R}_{+,-}^{n} \cup B_{1}^{n-1}(0)=\mathbb{R}^{n} \backslash C_{1, \infty}\right)$.

We want to point out that from the analysis we carried on in the case $\ell=0$ and in particular from

$$
0<R^{(0)}=\lim _{j \rightarrow+\infty} \frac{r_{j}^{n-p}}{\delta_{j} \varepsilon_{j}^{n-1}}
$$

we recovered both the critical sizes founded in [2] and correspondent to the two cases $\delta_{j}=1$ and $\delta_{j}=\varepsilon_{j}$.

Moreover we want to show that $\varphi=\varphi^{(0)}$. We have to check only the inequality $\varphi \leq \varphi^{(0)}$, the other one being obvious.

For any fixed $\eta>0$ let $\zeta \in W_{\text {loc }}^{1, p}\left(\mathbb{R}^{n} \backslash C_{1, \infty} ; \mathbb{R}^{m}\right)$ be such that $\zeta-z \in L^{p}\left(0,+\infty ; L^{p^{*}}\left(\mathbb{R}^{n-1} ; \mathbb{R}^{m}\right)\right)$, $\zeta \in L^{p}\left(-\infty, 0 ; L^{p^{*}}\left(\mathbb{R}^{n-1} ; \mathbb{R}^{m}\right)\right), D \zeta \in L^{p}\left(\mathbb{R}^{n} \backslash C_{1, \infty} ; \mathbb{R}^{m \times n}\right)$ and

$$
\int_{\mathbb{R}^{n} \backslash C_{1, \infty}} g(D \zeta) d x \leq \varphi^{(0)}(z)+\eta .
$$

For every $N>2$ we denote by $B_{N}$ the $n$-dimensional ball of radius $N$ centered in zero and by $B_{N}^{ \pm}$the set of the points $x \in B_{N}$ such that $\pm x_{n}>0$; we consider a cut-off function $\theta_{N} \in \mathcal{C}_{c}^{\infty}\left(B_{N} ;[0,1]\right)$ such that $\theta_{N}=1$ in $B_{N / 2},\left|D \theta_{N}\right| \leq c / N$ and we define

$$
\bar{\zeta}:=\left\{\begin{array}{lll}
\theta_{N}(\zeta-z)+z & \text { in } & B_{N}^{+}, \\
\theta_{N} \zeta & \text { in } & B_{N}^{-} \cup\left(B_{1}^{n-1} \times\{0\}\right)
\end{array}\right.
$$

so that $\bar{\zeta} \in W^{1, p}\left(B_{N} \backslash C_{1, N} ; \mathbb{R}^{m}\right), \bar{\zeta}=z$ on $\partial B_{N}^{+}$and $\bar{\zeta}=0$ on $\partial B_{N}^{-}$. Hence,

$$
\int_{B_{N} \backslash C_{1, N}} g(D \bar{\zeta}) d x=\int_{B_{N / 2} \backslash C_{1, N / 2}} g(D \zeta) d x+\int_{\left(B_{N} \backslash B_{N / 2}\right) \backslash C_{1, N}} g(D \bar{\zeta}) d x ;
$$

in particular, by $(7.2)$, we have

$$
\begin{aligned}
\int_{\left(B_{N} \backslash B_{N / 2}\right) \backslash C_{1, N}} g(D \bar{\zeta}) d x \leq & \beta\left(\int_{B_{N}^{+} \backslash B_{N / 2}^{+}}\left|D \theta_{N}\right|^{p}|\zeta-z|^{p} d x+\int_{B_{N}^{-} \backslash B_{N / 2}^{-}}\left|D \theta_{N}\right|^{p}|\zeta|^{p} d x\right. \\
& \left.+\int_{\left(B_{N} \backslash B_{N / 2} \backslash C_{1, N}\right.}|D \zeta|^{p} d x\right) \\
\leq & \frac{c}{N^{p}}\left(\int_{B_{N}^{+} \backslash B_{N / 2}^{+}}|\zeta-z|^{p} d x+\int_{B_{N}^{-} \backslash B_{N / 2}^{-}}|\zeta|^{p} d x\right) \\
& +\int_{\left(\mathbb{R}^{n} \backslash B_{N / 2}\right) \backslash C_{1, \infty}}|D \zeta|^{p} d x .
\end{aligned}
$$

Since $\zeta-z \in L^{p}\left(0,+\infty ; L^{p^{*}}\left(\mathbb{R}^{n-1} ; \mathbb{R}^{m}\right)\right), \zeta \in L^{p}\left(-\infty, 0 ; L^{p^{*}}\left(\mathbb{R}^{n-1} ; \mathbb{R}^{m}\right)\right)$ and $D \zeta \in L^{p}\left(\mathbb{R}^{n} \backslash C_{1, \infty} ; \mathbb{R}^{m \times n}\right)$, we can easily conclude that

$$
\lim _{N \rightarrow+\infty} \int_{\left(B_{N} \backslash B_{N / 2}\right) \backslash C_{1, N}} g(D \bar{\zeta}) d x=0 .
$$


Hence, by (7.40), we deduce

$$
\begin{aligned}
& \varphi^{(0)}(z)+\eta \geq \int_{\mathbb{R}^{n} \backslash C_{1, \infty}} g(D \zeta) d x \geq \int_{B_{N / 2} \backslash C_{1, N / 2}} g(D \zeta) d x \\
&=\int_{B_{N} \backslash C_{1, N}} g(D \bar{\zeta}) d x+o(1) \\
& \geq \inf \left\{\int_{B_{N} \backslash C_{1, N}} g(D \zeta) d x: \zeta \in W^{1, p}\left(B_{N} \backslash C_{1, N} ; \mathbb{R}^{m}\right)\right. \\
&\left.\zeta=z \text { on } \partial B_{N}^{+}, \zeta=0 \text { on } \partial B_{N}^{-}\right\}+o(1)
\end{aligned}
$$

as $N \rightarrow+\infty$. Finally, passing to the limit as $N \rightarrow+\infty$, by the arbitrariness of $\eta$, we get $\varphi^{(0)} \geq \varphi$.

Note that the proof of the explicit formula for $\varphi$ in [2] relies on the fact that $\delta_{j}$ is of order $\varepsilon_{j}$ or bigger than it, while in Proposition 7.11 and Proposition 7.12 we have to take into account that $\delta_{j} \ll \varepsilon_{j}$. This is the reason why our proof is different from the one of [2] even if, at the end, the two representation formulas turn out to coincide.

\section{Conclusions}

Our paper deals with the characterization of the effective energy of weakly connected thin structures through a periodically distributed contact zone. We highlight the presence of three different regimes (depending on the mutual rate of convergence of the radii of the connecting zones and the thickness of the domain) and for each of them we derive the limit energy by a $\Gamma$-convergence procedure. For each regime an interfacial energy term, depending on the jump of the deformation at the interface, appears in the limit representing the asymptotic memory of the sieve. We completely describe the interfacial energy densities by nonlinear capacitary type formulas.

Acknowledgments. The authors wish to thank Andrea Braides for suggesting the problem, for fruitful comments and stimulating discussions.

The research of J.-F. Babadjian has been supported by the Marie Curie Research Training Network MRTN-CT-2004-505226 'Multi-scale modelling and characterisation for phase transformations in advanced materials' (MULTIMAT).

\section{REFERENCES}

[1] R. A. Adams: Sobolev spaces, Academic Press, New York (1975).

[2] N. Ansini: The nonlinear sieve problem and applications to thin films, Asymptotic Analysis 39 (2004) $113-145$.

[3] N. Ansini and A. Braides: Asymptotic analysis of periodically-perforated nonlinear media, J. Math. Pures Appl. 81 (2002) 439-451.

[4] N. Ansini and A. Braides: Erratum to 'Asymptotic analysis of periodically-perforated nonlinear media', J. Math. Pures Appl. 84 (2005), no. 1, 147-148.

[5] H. Attouch and C. Picard: Comportement limite de problèmes de transmission unilatéraux à travers des grilles de forme quelconque, Rend. Sem. Mat. Univers. Politecn. Torino 45 (1987) 71-85.

[6] K. Bhattacharya, I. Fonseca and G. Francfort: An asymptotic study of the debonding of thin films, Arch. Rational Mech. Anal. 161 (2002) 205-229.

[7] K. Bhattacharya and R. D. James: A theory of thin films of martensitic materials with applications to microactuators, J. Mech. Phys. Solids 47 (1999) 531-576.

[8] M. Bocea and I. Fonseca: Equi-integrability results for 3D-2D dimension reduction problems, ESAIM: COCV 7 (2002) 443-470.

[9] A. Braides: Г-convergence for beginners, Oxford University Press, Oxford (2002).

[10] A. Braides and A. Defranceschi: Homogenization of multiple integrals, Oxford University Press, New York (1998).

[11] A. Braides, A. Defranceschi and E. Vitali: Homogenization of free discontinuity problems, Arch. Rational Mech. Anal. 135 (1996) 297-356.

[12] A. Braides and I. Fonseca: Brittle thin films, Appl. Math. Optim. 44 (2001), no. 3, 299-323.

[13] A. Braides, I. Fonseca and G. A. Francfort: 3D-2D asymptotic analysis for inhomogeneous thin films, Indiana Univ. Math. J. 49 (2000) 1367-1404. 
[14] A. Braides and C. I. Zeppieri: A note on equi-integrability in dimension reduction problems, Calc. Var. Partial Differential Equations, to appear.

[15] D. Cioranescu and F. Murat: Un terme étrange venu d'ailleurs, I and II. Nonlinear Partial Differential Equations and Their Applications. Collège de France Seminar. Vol. II, 98-138, and Vol. III, 154-178, Res. Notes in Math., 60 and 70, Pitman, London, 1982 and 1983.

[16] C. Conca: On the application of the homogenization theory to a class of problems arising in fluid mechanics, J. Math. Pures Appl. 64 (1985) 31-75.

[17] C. Conca: Étude d'un fluide traversant une paroi perforée I. Comportement limite près de la paroi, J. Math. Pures Appl. 66 (1987) 1-43.

[18] C. Conca: Étude d'un fluide traversant une paroi perforée II. Comportement limite loin de la paroi, J. Math. Pures Appl. 66 (1987) 45-69.

[19] B. Dacorogna: Direct methods in the calculus of variations, Springer-Verlag, Berlin (1989).

[20] G. Dal Maso: An Introduction to $\Gamma$-convergence, Birkhäuser, Boston (1993).

[21] A. Damlamian: Le problème de la passoire de Neumann, Rend. Sem. Mat. Univ. Politec. Torino, 43 (1985) $427-450$.

[22] T. Del Vecchio: The thick Neumann's sieve, Ann. Mat. Pura Appl. 147 (1987) 363-402.

[23] H. Le Dret and A. Raoult: The nonlinear membrane model as variational limit of nonlinear three-dimensional elasticity, J.Math. Pures Appl. 74 (1995) 549-578.

[24] F. Murat: The Neumann sieve, Nonlinear Variational Problems, A. Marino et coll., Res. Notes in Math. 127, Pitman, London (1985) 24-32.

[25] C. Picard: Analyse limite d'équations variationnelles dans un domaine contenant une grille, RAIRO Modél. Math. Anal. Numér. 21 (1987) 293-326.

[26] E. Sanchez-Palencia: Non-homogeneous media and vibration theory, Lecture Notes in Physics 127 Springer-Verlag, Berlin (1980).

[27] E. Sanchez-Palencia: Boundary value problems in domains containing perforated walls, Nonlinear Partial Differential Equations and Their Applications. Collège de France Seminar. Vol III, 309-325, Res. Notes in Math., 70, Pitman, London (1981).

[28] E. Sanchez-Palencia: Un problème d'écoulement lent d'un fluide visqueux incompressible au travers d'une paroi finement perforée, in D. Bergman et coll., Les Méthodes de l'Homogénéisation: Théorie et Applications en Physique, Collection de la Direction des Études et Recherches d'Électricité de France, 57 (1985) 371-400, Eyrolles, Paris.

[29] Y.C. Shu: Heterogeneous thin films of martensitic materials, Arch. Rational Mech. Anal. 153 (2000), no. 1, 39-90.

[30] K. Yosida: Functional Analysis, Springer Verlag, Berlin (1965).

(Nadia Ansini ${ }^{1}$ ) Dipartimento di Matematica 'G. Castelnuovo' Università di Roma 'La Sapienza', Piazzale Aldo Moro, 2, 00185 Rome, Italy

E-mail address: ansini@mat.uniroma1.it

(Jean-François Babadjian) SISSA, Via Beirut 2-4, 34014 Trieste, Italy

E-mail address: babadjia@sissa.it

(Caterina Ida Zeppieri) Dipartimento di Matematica 'G. Castelnuovo' Università di Roma 'La Sapienza', Piazzale Aldo Moro, 2, 00185 Rome, Italy

E-mail address: zeppieri@mat.uniroma1.it

\footnotetext{
${ }^{1}$ Present Address: Section de Mathématiques, EPFL, 1015 Lausanne, Switzerland. E-mail: nadia.ansini@epfl.ch
} 